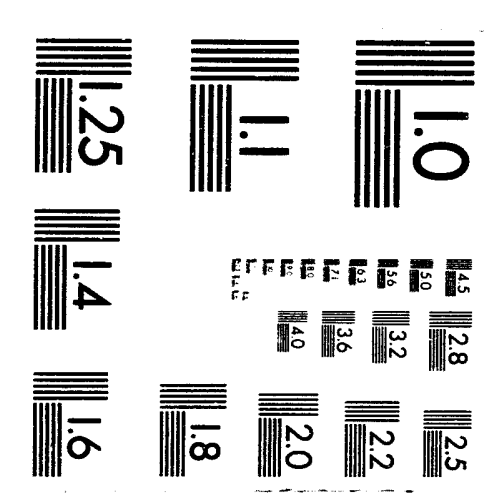





\section{INSTITUTE FOR RECEIVED
APR 11 MPC4 FUSION STUDIES \\ OSTI}

DE-FG05-80E T-53088-649

IFSR \#649

Stability of Coupled Tearing and

Twisting Modes in Tokamaks

RichaRd FitzPatrick

Institute for Fusion Studies

The University of Texas at Austin

Austin, Texas 78712

March 1994

THE UNIVERSITY OF TEXAS

AUSTIN 


\title{
Stability of Coupled Tearing and Twisting Modes in Tokamaks
}

\author{
RICHARD FITZPATRICK \\ Institute for Fusion Studies \\ The University of Texas at Austin \\ Austin, Texas 78712
}

\begin{abstract}
A dispersion relation is derived for resistive modes of arbitrary parity in a tokamak plasma. At low mode amplitude, tearing and twisting modes which have nonideal MHD behavior at only one rational surface at a time in the plasma are decoupled via sheared rotation and diamagnetic flows. At higher amplitude, more unstable 'compound' modes develop which have nonideal behavior simultaneously at many surfaces. Such modes possess tearing parity layers at some of the nonideal surfaces, and twisting parity layers at others, but mixed parity layers are generally disallowed. At low mode number, 'compound' modes are likely to have tearing parity layers at all of the nonideal surfaces in a very low- $\beta$ plasma, but twisting parity layers become more probable as the plasma $\beta$ is increased. At high mode number, unstable twisting modes which exceed a critical amplitude drive conventional magnetic island chains on alternate rational surfaces, to form an interlocking structure in which the O-points and X-points of neighboring chains line up.
\end{abstract}




\section{Introduction}

The study of resistive instabilities in tokamak plasmas is important because long wavelength resistive modes are thought to be responsible for current terminating disruptions, ${ }^{1}$ which are of particular concern in the ongoing International Tokamak Experimental Reactor (ITER) Engineering Design Activity, ${ }^{2}$ whereas short wavelength resistive modes may give rise to stochastic magnetic field lines and, hence, anomalous transport of energy and momentum. ${ }^{3}$

In a recent paper, Fitzpatrick, Hastie, Martin, and Roach ${ }^{4}$ (henceforth referred to as FHMR) investigated the stability of coupled long wavelength resistive modes in a tokamak plasma. It was found that differential rotation of rational surfaces in a high temperature device effectively diecouples low amplitude modes, so that they only reconnect magnetic flux at one surface in the plasma, and behave ideally at the remaining surfaces. However, above a threshold mode amplitude, the rational surfaces start to 'lock' together, permitting modes to develop which simultaneously reconnect flux at more than one surface. Such modes are always more unstable than the uncoupled modes.

The analysis of FHMR is restricted to the study of tearing parity modes, for which the perturbed normal magnetic field is even across resonant layers. In principle, however, there is no reason why modes of the opposite parity - so-called twisting modes - should not also be present. The stability of twisting parity modes is usually studied using the well-known ballooning transformation. ${ }^{15}$ Unfortunately, the resulting analysis, which takes place in an abstract 'ballooning space,' is only valid at very short wavelengths and is also difficult to reconcile with the conventional analysis of coupled tearing modes, which takes place in real space. Recently, however, Connor, Hastie, and Taylor ${ }^{6}$ (henceforth referred to as CHT) have shown how ballooning analysis for low- $\beta$ resistive modes can be performed in real space, and have developed a formalism which is valid at arbitrary wavelength. 
The aim of this paper is to combine the analyses of FHMR and CHT and thereby investigate the stability of resistive modes of arbitrary parity in a differentially rotating tokamak plasma. It is of particular interest to ascertain under what circumstances the conventional approach of neglecting twisting parity modes at long wavelengths, ${ }^{7}$ and tearing parity modes at short wavelengths, ${ }^{3}$ is valid.

\section{The Dispersion Relation for Coupled Tearing and Twisting Modes}

\section{A. Introduction}

The analysis of resistive instabilities in a high temperature tokamak is generally facilitated by dividing the plasma into two regions. ${ }^{8}$ In the 'outer' region, which comprises most of the plasma, a general instability is governed by the equations of ideal magnetohydrodynamics (MHD), which are equivalent to the requirement of force balance in an incompressible, perfectly conducting fluid. ${ }^{9}$ The 'inner' region is localized around so-called rational flux surfaces, where the helical pitch of equilibrium magnetic field lines resonates with that of the instability. The ideal-MHD equations are, in fact, singular at the rational surfaces. The physical solution is obtained by asymptotically matching the outer solution across a set of thin layers centered on the rational surfaces. In these layers nonideal effects such as plasma resistivity, inertia, viscosity, and compressibility are important.

In the immediate vicinity of a nonideal layer the instability is conveniently described in terms of the resonant plasma displacement $\phi(x)$ and perturbed poloidal magnetic flux $\psi(x)$, where $x$ is the radial distance from the rational surface (see Appendix B). Most layer equations are parity conserving; i.e. they are invariant under the transformation $x \rightarrow-x$, $\phi \rightarrow \pm \phi, \psi \rightarrow \mp \psi$. This implies that the twisting parity mode $[\phi(-x)=\phi(x), \psi(-x)=$ $-\psi(x)]$ completely decouples from the tearing parity mode $[\phi(-x)=-\phi(x), \psi(-x)=\psi(x)]$ inside the layer. However, the ideal-MHD equations in the outer region are not parity 
conserving because of radial gradients in the equilibrium plasma current and pressure, as well as the underlying toroidal geometry. This leads to the coupling of tearing and twisting modes in the outer region. Moreover, toroidicity and flux surface shaping lead to the coupling of different poloidal harmonics in the outer region. Thus, asymptotic matching between the inner and outer regions is a complicated procedure which involves the simultaneous mat,ching of many poloidal harmonics in the outer region to tearing and twisting parity layer solutions at the various rational surfaces in the plasma.

The layer equations are most easily solved in Fourier transform space. ${ }^{10}$ Let $\hat{\phi}(k)$ be the Fourier transform of the resonant plasma displacement $\phi(x)$ for a particular layer. The parity conserving properties of the layer allow $\hat{\phi}(k)$ to be split into independent even (twisting) and odd (tearing) parity components:

$$
\widehat{\phi}(k)=\frac{1}{2} \hat{\phi}^{+}(|k|)-\frac{\mathrm{i}}{2} \hat{\phi}^{-}(|k|) \operatorname{sgn}(k) .
$$

The most general small- $k$ asymptotic behavior of the transformed displacement is written ${ }^{6}$

$$
\begin{aligned}
\hat{\phi}^{+}(|k|) & \simeq \Psi^{-}\left[\frac{1}{\pi} \Delta^{-}(\omega)|k|^{\nu-1}+|k|^{-\nu}+\cdots\right] \\
\hat{\phi}^{-}(|k|) & \simeq \Psi^{+}\left[\frac{1}{\pi} \Delta^{+}(\omega)|k|^{\nu-1}+|k|^{-\nu}+\cdots\right] .
\end{aligned}
$$

Here, $\nu$ represents the effect of average field-line curvature in the vicinity of the layer; it is related to the well-known Mercier stability criterion $\frac{1}{4}-\nu>0 .{ }^{11}$ The parameter $\Delta^{+}(\omega)$ is termed the stability index for tearing parity modes, and is entirely determined by the solution of the tearing parity la sr equations. In general, it is a function of the mode rotation frequency $\omega$. Likewise, $\Delta^{-}(\omega)$ is the stability index for twisting parity modes, determined by the solution of the twisting parity layer equations.

Equations (2) can be inverted to give the asymptotic behavior of the layer solution as it merges into the outer region:

$$
\phi(x)=\phi^{+}(|x|)+\phi^{-}(|x|) \operatorname{sgn}(x)
$$


where

$$
\begin{aligned}
& \phi^{+}(|x|) \simeq \Psi^{-}\left[\left(\frac{\nu \pi}{2}\right)|x|^{\nu-1}+\frac{1}{2}\left(\frac{\nu \pi}{2}\right)^{-1} \Lambda^{-}(\omega)|x|^{-\nu}+\cdots\right]+\mathcal{C}, \\
& \phi^{-}(|x|) \simeq \Psi^{+}\left[|x|^{\nu-1}+\frac{1}{2} \Delta^{+}(\omega)|x|^{-\nu}+\cdots\right],
\end{aligned}
$$

for $|\nu| \ll 1$. In the above, $\Psi^{+}$is termed the 'tearing amplitude' and $\Psi^{-}$the 'twisting amplitude.' The parameter $\mathcal{C}$ is an arbitrary constant. In the outer region $\psi=x \phi$, so

$$
\psi(x)=\psi^{+}(|x|)+\psi^{-}(|x|) \operatorname{sgn}(x),
$$

where

$$
\begin{aligned}
& \psi^{+}(|x|) \simeq \Psi^{+}\left[|x|^{\nu}+\frac{1}{2} \Delta^{+}(\omega)|x|^{1-\nu}+\cdots\right] \\
& \psi^{-}(|x|) \simeq \Psi\left[\left(\frac{\nu \pi}{2}\right)|x|^{\nu}+\frac{1}{2}\left(\frac{\nu \pi}{2}\right)^{-1} \Delta^{-}(\omega)|x|^{1-\nu}+\cdots\right]+\mathcal{C}|x| .
\end{aligned}
$$

In a low- $\beta$, large aspect-ratio tokamak the Mercier index $\nu$ is $\mathcal{O}\left(\epsilon^{2}\right)$, where $\epsilon \ll 1$ is the ratio of the minor and major radii of the plasma. In the limit $\nu \rightarrow 0$, Eqs. (4) and (6) yield

$$
\begin{aligned}
& \phi^{+}(|x|) \simeq \Psi^{-}\left[\pi \delta(|x|)-\frac{1}{\pi} \Delta^{-}(\omega) \ln |x|+\cdots\right]+\mathcal{C}^{\prime}, \\
& \phi^{-}(|x|) \simeq \Psi^{+}\left[\frac{1}{|x|}+\frac{1}{2} \Delta^{+}(\omega)+\cdots\right],
\end{aligned}
$$

and

$$
\begin{aligned}
& \psi^{+}(|x|) \simeq \Psi^{+}\left[1+\frac{1}{2} \Delta^{+}(\omega)|x|+\cdots\right] \\
& \psi^{-}(|x|) \simeq \Psi^{-}\left[-\frac{1}{\pi} \Delta^{-}(\omega)|x| \ln |x|+\cdots\right]+\mathcal{C}^{\prime}|x| .
\end{aligned}
$$

Thus, in the zero-curvature limit the tearing stability index $\Delta^{+}(\omega)$ corresponds to the jump in logarithmic derivative of $\psi(x)$ across the layer. ${ }^{8}$ The twisting parity stability index can be written ${ }^{6}$

$$
\Delta^{-}(\omega)=-\frac{\pi^{2} \times \text { coeff. } \ln |x|}{\text { coeff. } \delta(|x|)}
$$


where the coefficients refer to the expansion of $\phi(x)$ in the vicinity of the layer. When added to the original plasma equilibrium, the tearing parity mode reconnects magnetic flux at the rational surface to produce a chain of magnetic islands. The X-points form where the odd parity displacement in (7b) is directed into the layer, and the O-points form where the displacement is directed out of the layer. The tearing amplitude $\Psi^{+}$is sometimes termed the 'reconnected flux' at the rational surface. Equations (7a) and (8b) imply that the twisting parity mode is essentially interchange-like; i.e. it is strongly localized inside the layer. The twisting amplitude $\Psi^{-}$is a measure of the localized even parity plasma displacement at the rational surface.

\section{B. The outer solution}

The physics of the outer region is discussed in detail in Appendix A. Suppose that there are $N$ rational surfaces in the plasma resonant with toroidal mode number $n$. Let $r_{1}<r_{2}<$ $\cdots r_{N}$ be the minor radii of these surfaces, and $m_{1}, m_{2}, \cdots m_{N}$ the resonant poloidal mode numbers. The most general dispersion relation for coupled tearing and twisting modes takes the form: $:^{7,12,13}$

$$
\begin{aligned}
\left\{\boldsymbol{\Delta}^{+}(\omega)-\mathbf{E}^{+}\right\} \boldsymbol{\Psi}^{+}-\mathbf{H} \boldsymbol{\Psi}^{-}=\mathbf{0} \\
\left\{\boldsymbol{\Delta}^{-}(\omega)-\mathbf{E}^{-}\right\} \boldsymbol{\Psi}^{-}-\mathbf{H}^{\dagger} \boldsymbol{\Psi}^{+}=\mathbf{0}
\end{aligned}
$$

where $\mathrm{E}^{ \pm}$is an $N \times N$ real symmetric matrix, $\mathbf{H}$ is an $N \times N$ real matrix and $\mathbf{H}^{\dagger}$ is its transpose, $\Delta^{ \pm}(\omega)$ is the $N \times N$ complex diagonal matrix of the $\Delta_{j}^{ \pm}(\omega)$ values, and $\Psi^{ \pm}$is the $1 \times N$ complex vector of the $\Psi_{j}^{ \pm}$values. Here, $\Delta_{j}^{+}(\omega)$ is the tearing parity stability index for the layer at rational surface $j$, and $\Delta_{j}^{-}(\omega)$ is the corresponding twisting parity stability index. Also, $\Psi_{j}^{+}$is the tearing amplitude at surface $j$, and $\Psi_{j}^{-}$the corresponding twisting amplitude.

The $\mathrm{E}^{+}$Matrix determines the intrinsic stability and mutual interaction of tearing parity 
modes in the plasma. In general, it can only be evaluated by solving the full coupled ideal-MHD equations in the outer region. This can be achieved for a large aspect-ratio, low- $\beta$, weakly shaped tokamak equilibrium using the recently developed T7 code. ${ }^{4}$ The basic T7 ordering assumptions are that the Shafranov shift and departure from circularity of flux surfaces are both $\mathcal{O}(\epsilon)$ with respect to the plasma minor radius. This implies that $\beta=,{ }^{\prime} p_{0} / B_{0}^{2} \sim \mathcal{O}\left(\epsilon^{2}\right)$, where $p_{0}$ is the central plasma pressure and $B_{0}$ the on-axis vacuum toroidalfield strength.

$\mathrm{C}$ asider a plasma with a monotonic safety factor profile containing no rational surfaces resonant with poloidal mode number $m=1$. (The restriction to $m>1$ modes is necessary because the $m=1$ mode generally requires special treatment in tokamak plasmas. ${ }^{14}$ ) In such a plasma the diagonal elements of the $\mathrm{E}^{+}$Matrix take the form

$$
E_{j j}^{+}=\Delta_{j}^{0}+\mathcal{O}\left(\epsilon^{2}\right),
$$

where $\Delta_{j}^{0}$ is the standard cylindrical tearing stability index for the $m_{j} / n$ mode (normalized with respect to $r_{j}$ ). The off-diagonal elements of the $\mathrm{E}^{+}$Matrix are $\mathcal{O}(\epsilon)$. Coupling of tearing parity modes with poloidal mode numbers differing by unity is effected by the Shafranov shift of flux surfaces, which is driven by toroidicity and the plasma pressure. Coupling of modes with poloidal mode numbers differing by two or three is effected by flux-surface ellipticity or triangularity, respectively.

The $E^{-}$Matrix determines the intrinsic stability and mutual interaction of twisting parity modes in the plasma. In Appendix $\mathrm{A}$ it is demonstrated that the ordering $\nu \sim \mathcal{O}\left(\epsilon^{2}\right)$ (which is consistent with the $\mathrm{T} 7$ ordering scheme) can be exploited to greatly simplify the evaluation of this matrix. This technique, first described in $\mathrm{CHT}^{6}{ }^{6}$ allows the elements of the matrix to be calculated using a combination of local equilibrium parameters evaluated at the rational surfaces and cylindrical basis functions. For a plasma with a monotonic safety factor profile the $\mathrm{E}^{-}$Matrix is diagonal, indicating that there is no direct coupling of twisting modes 
possessing different poloidal mode numbers. The $j$ th diagonal element can be written

$$
E_{j j}^{-}=e_{j} m_{j}\left(\alpha_{j}\right)^{2}
$$

where

$$
\alpha_{j}=-\left(\frac{2 R_{0} \mu_{0} p^{\prime} q^{2}}{B_{0}^{2}}\right)_{r_{j}}
$$

is a measure of the local pressure gradient at rational surface $j$. Here, $R_{0}$ is the major radius of the plasma, $r$ the minor radius of flux surfaces, $p(r)$ the equilibrium pressure profile, $q(r)$ the safety factor profile, and ' denotes $d / d r$ (see Sec. A.I). Note that $\alpha_{j} \sim \mathcal{O}(\epsilon)$ in the $\mathrm{T} 7$ ordering scheme. In Eq. (12), $e_{j}$ is an $\mathcal{O}(1)$ parameter which can be evaluated using $m_{j} \pm 1 / n$ cylindrical basis functions (see Sec. A.VI). In the cylindrical limit, $\epsilon \rightarrow 0$, the diagonal elements of the $\mathrm{E}^{-}$Matrix asymptote to zero. This indicates that in the outer region twisting parity modes are intrinsically toroidal in nature. ${ }^{6}$ In fact, unit twisting amplitude at rational surface $j$ drives $\mathcal{O}(\epsilon)$ of the toroidally coupled sidebands (with poloidal mode numbers $m_{j} \pm 1$ ) in the outer region, but only $\mathcal{O}\left(\epsilon^{2}\right)$ of the resonant harmonic (with poloidal mode number $m_{j}$ ) (see Sec. A.VI).

The $\mathrm{H}$ Matrix determines the mutual interaction of tearing and twisting parity modes in the plasma. The ordering $\nu \sim \mathcal{O}\left(\epsilon^{2}\right)$ can again be exploited to simplify the evaluation of this matrix (see Appendix A). For a plasma with a monotonic safety factor profile the H Matrix is tridiagonal, indicating that tearing modes can couple to twisting modes with the same poloidal mode number and also with mode numbers differing by unity, and vice versa. The $j$ th diagonal element is written

$$
H_{j j}=-\frac{\pi}{2} \frac{\kappa_{j}}{s_{j}}+\mathcal{O}\left(\epsilon^{2}\right)
$$

where

$$
\kappa_{j}=\left(-q r \frac{d}{d r}\left[\frac{1}{r} \frac{d}{d r}\left(\frac{r^{2}}{q}\right)\right]\right)_{r_{j}}+\mathcal{O}\left(\epsilon^{2}\right)
$$


is a measure of the local equilibrium current gradient at rational surface $j$, and $s_{j}=\left(r q^{\prime} / q\right)_{r_{j}}$ is the local magnetic shear. The element of the $\mathrm{H}$ Matrix which couples the tearing mode resonant at rational surface $j$ to the twisting mode resonant at surface $k$ takes the form

$$
H_{j k}=h_{j k} m_{j} \alpha_{k}
$$

provided $m_{k}=m_{j} \pm 1$. The $\mathcal{O}(1)$ parameter $h_{j k}$ can be evaluated using $m_{j} / n$ cylindrical basis functions (see Sec. A.V).

\section{The inner solution}

The physics of the inner region is discussed in detail in Appendix B. The basic aim of this study is to derive the simplest possible expressions for the tearing and twisting parity layer dispersion relations which retain certain fundamental pieces of physics. For instance, plasma perpendicular viscosity is considered to play a very significant role in resonant layers, so much of Appendix B is devoted to a systematic study of the effect of viscosity on single-fluid layer physics (see Sec. B.I). Plasma compressibility is particularly important because it differentiates between tearing and twisting parity layers and is, of course, a vital element in the physics of twisting parity interchange modes (see Sec. B.II). Plasma differential rotation and multi-fluid effects are even more important because they cause twisting and tearing modes to resonate at different frequencies, thereby profoundly affecting their mutual interaction ${ }^{15}$ (see Sec. B.III). Many other effects, such as field-line curvature, ${ }^{16}$ semi-collisionality, ${ }^{17}$ and trapped particles, ${ }^{18}$ which are not considered to play a vital role in the mutual interactions of tearing and twisting parity modes, are neglected for the sake of clarity.

According to Appendix $\mathrm{B}$, the tearing parity layer dispersion relation at rational surface $j$ takes the form

$$
\Delta_{j}^{+}(\omega)=-\mathrm{i}\left(\omega-\omega_{j}^{+}\right) \tau_{j},
$$


and the associated twisting parity dispersion relation is well approximated by

$$
\Delta_{j}^{-}(\omega)=-\mathrm{i}\left(\omega-\omega_{j}^{-}\right) \tau_{j}+\Delta_{j}^{c}
$$

where

$$
\tau_{j}=2.104\left(\frac{\tau_{H}^{1 / 3} \tau_{R}^{5 / 6}}{\tau_{V}^{1 / 6}}\right)_{r_{j}}
$$

and

$$
\Delta_{j}^{c}=2.104 \beta_{j}^{1 / 2}\left(\frac{\tau_{R}^{2 / 3}}{\tau_{H}^{1 / 3} \tau_{V}^{1 / 3}}\right)_{r_{j}} .
$$

In the above, $\tau_{H}(r)=\left(R_{0} / B_{0}\right) \sqrt{\mu_{0} \rho(r)} / n s(r)$ is the hydromagnetic timescale, $\tau_{R}(r)=$ $\mu_{0} r^{2} / \eta_{\|}(r)$ the resistive timescale, $\tau_{V}(r)=r^{2} \rho(r) / \mu_{\perp}(r)$ the viscous timescale, and $\tau_{j}$ the reconnection timescale at surface $j$. Here, $\rho(r)$ is the plasma mass density, $\eta_{\|}(r)$ the parallel resistivity, $\mu_{\perp}(r)$ the perpendicular viscosity, and $s(r)=r q^{\prime} / q$ the magnetic shear. The parameter $\beta_{j}=\gamma_{s} \mu_{0} p\left(r_{j}\right) / B_{0}^{2}$ is a measure of the stabilizing effect of plasma compressibility at surface $j$, where $\gamma_{s}$ is the standard ratio of specific heats. In Eqs. (17) and (18), $\omega$ is the mode rotation frequency [all layer quantities are assumed to vary like $\exp (-i \omega t)$ ], $\omega_{j}^{+}$is termed the natural frequency for tearing parity modes at surface $j$, and $\omega_{j}^{-}$is the corresponding natural frequency for twisting parity modes. Both natural frequencies are determined by local equilibrium plasma flows. Broadly speaking, $\omega_{j}^{+}$is the sum of the $\mathbf{E} \wedge \mathbf{B}$ and electron diamagnetic frequencies at surface $j$, while $\omega_{j}^{-}$is the $\mathbf{E} \wedge \mathbf{B}$ frequency. The above dispersion relations are valid provided

$$
\begin{gathered}
\left|\omega-\omega_{j}^{ \pm}\right| \ll \omega_{j}^{(1)} \equiv\left(\frac{\tau_{R}^{1 / 3}}{\tau_{H}^{2 / 3} \tau_{V}^{2 / 3}}\right)_{r_{j}}, \\
\left|\omega-\omega_{j}^{ \pm}\right| \ll \omega_{j}^{(2)} \equiv\left(\frac{\tau_{V}^{1 / 3}}{\tau_{H}^{2 / 3} \tau_{R}^{2 / 3}}\right)_{r_{j}}, \\
\beta_{j} \ll\left(\frac{\tau_{R}}{\tau_{V}}\right)_{r_{j}} \ll \frac{1}{\beta_{j}} .
\end{gathered}
$$


In Eq. (18), $\Delta_{j}^{c}$ is the critical twisting parity stability index required to overcome the stabilizing effect of plasma compressibility in the $j$ th layer. It is convenient to regard $-\Delta_{j}^{c}$ as the cylindrical part of the outer matrix element $E_{j j}^{-}$, so that

$$
\begin{aligned}
& \Delta_{j}^{-}(\omega) \rightarrow \Delta_{j}^{-}(\omega)-\Delta_{j}^{c}=-\mathrm{i}\left(\omega-\omega_{j}^{-}\right) \tau_{j}, \\
& E_{j j}^{-}(\omega) \rightarrow E_{j j}^{-}(\omega)-\Delta_{j}^{c}=-\Delta_{j}^{c}+e_{j} m_{j}\left(\alpha_{j}\right)^{2}
\end{aligned}
$$

[see Eq. (12)]. The twisting parity dispersion relation (10b) is invariant under the above transformation. According to this reformulation of the dispersion relation, the layer response of twisting modes [i.e. Eq. (22a)] is analogous to that of tearing modes [i.e. Eq. (17)], apart from the difference in natural frequencies. Note also the similarity of Eqs. (11) and (22b). Clearly, in the cylindrical limit twisting modes act very much like stable tearing modes. The cylindrical part of the twisting parity stability index (22b) is determined in the inner, rather than the outer, region because in a cylinder twisting modes are entirely localized within the various resonant layers.

\section{Electromagnetic and viscous torques}

The nonlinear toroidal electromagnetic torque acting at rational surface $j$ is given by

$$
\delta T_{\phi \mathrm{EM}}\left(r_{j}\right)=\frac{2 n \pi^{2} R_{0}}{\mu_{0}} \times\left[\operatorname{Im}\left(\Delta_{j}^{+}\right)\left|\Psi_{j}^{+}\right|^{2}+\operatorname{Im}\left(\Delta_{j}^{-}\right)\left|\Psi_{j}^{-}\right|^{2}\right]
$$

(see Sec. A.IV). Note that according to Eqs. (10)

$$
T_{\phi \mathrm{EM}}=\sum_{j=1}^{N} \delta T_{\phi \mathrm{EM}}\left(r_{j}\right)=\frac{2 n \pi^{2} R_{0}}{\mu_{0}} \times \sum_{j, k=1}^{N}\left[E_{j k}^{+}\left(\Psi_{j}^{+}\right)^{*} \Psi_{k}^{+}+E_{j k}^{-}\left(\Psi_{j}^{-}\right)^{*} \Psi_{k}^{-}\right]=0,
$$

since $\mathbf{E}^{+}$and $\mathbf{E}^{-}$are symmetric, so there is zero net electromagnetic torque acting on the plasma.

The electromagnetic torques which develop in the plasma modify the bulk toroidal rotation. ${ }^{4,19.20}$ (It is assumed that any modifications to the bulk poloidal rotation are prevented by strong poloidal flow damping.) Such modifications are opposed by the action of 
perpendicular plasma viscosity. For a steady-state plasma, the change in the toroidal angular rotation velocity $\Omega_{\phi}(r)$ satisfies $^{4,19,20}$

$$
\frac{d}{d r}\left[\left(r \mu_{\perp}\right) \frac{d \Omega_{\phi}}{d r}\right]=0 .
$$

The toroidal rotation of the plasma is assumed to be 'clamped' at the edge $(r=a),{ }^{19,21}$ so that $\Omega_{\phi}(a)=0$. The viscous restoring torque which develops in the vicinity of rational surface $j$ is written

$$
\delta T_{\phi \mathrm{VS}}\left(r_{j}\right)=4 \pi^{2} R_{0} \times\left[\left(r \mu_{\perp} R_{0}^{2}\right) \frac{d \Omega_{\phi}}{d r}\right]_{r_{j-}}^{r_{j+}} .
$$

In a steady-state plasma the viscous and electromagnetic torques must balance at every rational surface, giving

$$
\delta T_{\phi \mathrm{EM}}\left(r_{j}\right)+\delta T_{\phi \mathrm{Vs}}\left(r_{j}\right)=0
$$

for $j=1$ to $N$. Finally, the changes induced in the plasma toroidal angular velocity profile Doppler shift the natural frequencies of the various resonant layers, so that

$$
\omega_{j}^{ \pm} \rightarrow \omega_{j}^{ \pm}-n \Omega_{\phi}\left(r_{j}\right)
$$

where $\omega_{j}^{ \pm}$now denotes the natural frequency in the unperturbed plasma.

\section{E. Summary}

Sections II.A to II.D describe the basic elements of the stability analysis for resistive modes of arbitrary parity in a tokamak plasma possessing sheared rotation and diamagnetic flows. In the following sections, these elements are employed to investigate the stability of both long wavelength and short wavelength modes. 


\section{Stability of a Plasma Containing a Single Rational Surface}

\section{A. Introduction}

Consider the stability of low- $n$ modes for which there are only a comparatively small number of rational surfaces in the plasma. The simplest possible situation is where there is only a single rational surface, radius $r_{1}$, resonant with poloidal mode number $m_{1}$. In this case the dispersion relation $(10)$ reduces to

$$
\frac{\Psi_{1}^{-}}{\Psi_{1}^{+}}=\frac{H_{11}}{-\mathrm{i}\left(\omega-\omega_{1}^{-}\right) \tau_{1}-E_{11}^{-}}=\frac{-\mathrm{i}\left(\omega-\omega_{1}^{+}\right) \tau_{1}-E_{11}^{+}}{H_{11}}
$$

with the aid of Eqs. (17) and (22a). Here, $\Psi_{1}^{+}$is the tearing amplitude at the rational surface, $\Psi_{1}^{-}$the twisting amplitude, $\tau_{1}$ the reconnection timescale, $\omega_{1}^{+}$the natural frequency of tearing parity modes, and $\omega_{1}^{-}$the natural frequency of twisting parity modes. Also,

$$
\begin{aligned}
& E_{11}^{+}=\Delta_{1}^{0}+\mathcal{O}\left(\epsilon^{2}\right), \\
& E_{11}^{-}=-\Delta_{1}^{c}+\mathcal{O}\left(\epsilon^{2}\right), \\
& H_{11}=-\frac{\pi}{2} \frac{\kappa_{1}}{s_{1}}+\mathcal{O}\left(\epsilon^{2}\right)
\end{aligned}
$$

[see Eqs. (11), (14), (22b)], where $\Delta_{1}^{0}$ is the $m_{1} / n$ cylindrical tearing stability index, $\Delta_{1}^{c}>0$ is a measure of the ideal-MHD free energy needed to destabilize the resistive interchange mode, $\kappa_{1}$ is proportional to the equilibrium current gradient at the rational surface, and $s_{1}$ is the local magnetic shear.

\section{B. Effect of diamagnetic flows}

The difference between the natural frequencies of tearing and twisting parity modes at the rational surface is of order the local electron diamagnetic frequency, implying that $\mid \omega_{1}^{+}-$ 
$\omega_{1}^{-} \mid \tau_{1} \gg 1$ in a typical high temperature tokamak plasma. ${ }^{15,19}$ In this limit, the dispersion relation (29) yields a predominantly tearing parity mode (i.e. $\left|\Psi_{1}^{-}\right| \ll\left|\Psi_{1}^{+}\right|$) with

$$
\omega \simeq \omega_{1}^{+}-\frac{\left(H_{11}\right)^{2}}{\left(\omega_{1}^{+}-\omega_{1}^{-}\right) \tau_{1}^{2}}+\mathrm{i} \frac{E_{11}^{+}}{\tau_{1}}
$$

and a predominantly twisting parity mode (i.e. $\left|\Psi_{1}^{+}\right| \ll\left|\Psi_{1}^{-}\right|$) with

$$
\omega \simeq \omega_{1}^{-}+\frac{\left(H_{11}\right)^{2}}{\left(\omega_{1}^{+}-\omega_{1}^{-}\right) \tau_{1}^{2}}+\mathrm{i} \frac{E_{11}^{-}}{\tau_{1}}
$$

The tearing parity modes rotates close to its natural frequency $\omega_{1}^{+}$, is unstable if $E_{11}^{+}>0$, stable if $E_{11}^{+}<0$, and evolves on the reconnection timescale $\tau_{1}$. The twisting parity mode rotates close to its natural frequency $\omega_{1}^{-}$, and is always stable, since $E_{11}^{-}<0$. Note that the current gradient coupling parameter $H_{11}$ gives rise to a small frequency shift such as to bring the rotation frequencies of the two modes closer together.

\section{Effect of no diamagnetic flows}

If the rather unphysical limit $\left|\omega_{1}^{+}-\omega_{1}^{-}\right| \tau_{1} \ll 1$ is adopted the dispersion relation (29) has two roots with

$$
\begin{aligned}
& \gamma \tau_{1}=\frac{1}{2}\left(E_{11}^{+}+E_{11}^{-}\right) \pm\left[\frac{1}{4}\left(E_{11}^{+}-E_{11}^{-}\right)^{2}+\left(H_{11}\right)^{2}\right]^{1 / 2}, \\
& \frac{\Psi_{1}^{-}}{\Psi_{1}^{+}}=H_{11} / \frac{1}{2}\left(E_{11}^{+}-E_{11}^{-}\right) \pm\left[\frac{1}{4}\left(E_{11}^{+}-E_{11}^{-}\right)^{2}+\left(H_{11}\right)^{2}\right]^{1 / 2},
\end{aligned}
$$

where $\omega=\omega_{1}^{ \pm}+\mathrm{i} \gamma$. Thus, if there is no substantial difference between the two natural frequencies, the tearing and twisting parity modes merge to form two hybrid modes. The 'tearing/twisting' hybrid [+ sign in (33)] has the larger tearing component, and is more unstable than either of the pure parity modes. The 'twisting/tearing' hybrid [- sign in (33)] has the smaller tearing component, and is more stable than either of the pure parity modes. 


\section{Conclusions}

The difference in the natural frequencies of tearing and twisting parity modes in a conventional tokamak plasma leads to the effective decoupling of the two parities, giving rise to a pure tearing parity mode whose stability is determined by the standard tearing stability index (i.e. $E_{11}^{+}$), and a pure twisting parity mode which is always stable at low- $n$. If there is no difference in natural frequencies, the tearing and twisting parity modes merge to form two hybrid modes with more complicated behavior.

\section{Stability of a Plasma Containing Two Rational Surfaces}

\section{A. Linear stability}

Consider a plasma containing two rational surfaces, radii $r_{1}$ and $r_{2}\left(r_{1}<r_{2}\right)$, resonant with poloidal mode numbers $m_{1}$ and $m_{1}+1$, respectively. In this case, the dispersion relation (10) reduces to

$$
\begin{aligned}
& -\mathrm{i}\left(\omega-\omega_{1}^{+}\right) \tau_{1} \Psi_{1}^{+}=E_{11}^{+} \Psi_{1}^{+}+E_{12}^{+} \Psi_{2}^{+}+H_{11} \Psi_{1}^{-}+H_{12} \Psi_{2}^{-}, \\
& -\mathrm{i}\left(\omega-\omega_{2}^{+}\right) \tau_{2} \Psi_{2}^{+}=E_{22}^{+} \Psi_{2}^{+}+E_{12}^{+} \Psi_{1}^{+}+H_{22} \Psi_{2}^{-}+H_{21} \Psi_{1}^{-}, \\
& -\mathrm{i}\left(\omega-\omega_{1}^{-}\right) \tau_{1} \Psi_{1}^{-}=E_{11}^{-} \Psi_{1}^{-}+H_{11} \Psi_{1}^{+}+H_{21} \Psi_{2}^{+}, \\
& -\mathrm{i}\left(\omega-\omega_{2}^{-}\right) \tau_{2} \Psi_{2}^{-}=E_{22}^{-} \Psi_{2}^{-}+H_{22} \Psi_{2}^{+}+H_{12} \Psi_{1}^{+}
\end{aligned}
$$

with the aid of Eqs. (17) and (22a). In the physically relevant limit where the four frequencies

$\omega_{1}^{ \pm}$and $\omega_{2}^{ \pm}$are well separated (i.e. $\left|\omega_{1}^{+}-\omega_{2}^{+}\right| \tau_{1} \gg 1$, etc.), Eqs. (34) possess the following four roots:

$$
\omega \simeq \omega_{1}^{+}-\frac{\left(H_{11}\right)^{2}}{\left(\omega_{1}^{+}-\omega_{1}^{-}\right) \tau_{1}^{2}}-\frac{\left(H_{12}\right)^{2}}{\left(\omega_{1}^{+}-\omega_{2}^{-}\right) \tau_{1} \tau_{2}}-\frac{\left(E_{12}^{+}\right)^{2}}{\left(\omega_{1}^{+}-\omega_{2}^{+}\right) \tau_{1} \tau_{2}}+\mathrm{i} \frac{E_{11}^{+}}{\tau_{1}}
$$




$$
\begin{aligned}
& \omega \simeq \omega_{2}^{+}-\frac{\left(H_{22}\right)^{2}}{\left(\omega_{2}^{+}-\omega_{2}^{-}\right) \tau_{2}^{2}}-\frac{\left(H_{21}\right)^{2}}{\left(\omega_{2}^{+}-\omega_{1}^{-}\right) \tau_{1} \tau_{2}}-\frac{\left(E_{12}^{+}\right)^{2}}{\left(\omega_{2}^{+}-\omega_{1}^{+}\right) \tau_{1} \tau_{2}}+\mathrm{i} \frac{E_{22}^{+}}{\tau_{2}}, \\
& \omega \simeq \omega_{1}^{-}-\frac{\left(H_{11}\right)^{2}}{\left(\omega_{1}^{-}-\omega_{1}^{+}\right) \tau_{1}^{2}}-\frac{\left(H_{21}\right)^{2}}{\left(\omega_{1}^{-}-\omega_{2}^{+}\right) \tau_{1} \tau_{2}}+\mathrm{i} \frac{E_{11}^{-}}{\tau_{1}} \\
& \omega \simeq \omega_{2}^{-}-\frac{\left(H_{22}\right)^{2}}{\left(\omega_{2}^{-}-\omega_{2}^{+}\right) \tau_{2}^{2}}-\frac{\left(H_{12}\right)^{2}}{\left(\omega_{2}^{-}-\omega_{1}^{+}\right) \tau_{1} \tau_{2}}+\mathrm{i} \frac{E_{22}^{-}}{\tau_{2}} .
\end{aligned}
$$

The eigenfunctions associated with these roots are

$$
\begin{aligned}
& \left|\Psi_{1}^{-}\right|,\left|\Psi_{2}^{ \pm}\right| \ll\left|\Psi_{1}^{+}\right|, \\
& \left|\Psi_{2}^{-}\right|, \quad\left|\Psi_{1}^{ \pm}\right| \ll\left|\Psi_{2}^{+}\right|, \\
& \left|\Psi_{1}^{+}\right|, \quad\left|\Psi_{2}^{ \pm}\right| \ll\left|\Psi_{1}^{-}\right|, \\
& \left|\Psi_{2}^{+}\right|, \quad\left|\Psi_{1}^{ \pm}\right| \ll\left|\Psi_{2}^{-}\right|,
\end{aligned}
$$

respectively. Thus, diamagnetic flows and sheared rotation give rise to a splitting of the two parities as well as a decoupling of the two rational surfaces, so that the four roots of the dispersion relation correspond to a tearing mode which only has finite tearing amplitude at surface 1 [Eqs. (35a), (36a)], a tearing mode which only has finite amplitude at surface 2 [Eqs. (35b), (36b)], a twisting mode which only has finite twisting amplitude at surface 1 [Eqs. (35c), (36c)], and a twisting mode which only has finite amplitude at surface 2 [Eqs. (35d), (36d)].

According to Eqs. (35) and (36), there is a pure tearing and a pure twisting parity mode associated with each rational surface in a plasma possessing sheared rotation and diamagnetic flows. The tearing mode associated with surface $j(j=1$ or 2$)$ only has finite tearing amplitude at this surface, rotates at the appropriate natural frequency $\left(\omega_{j}^{+}\right)$, has its stability determined by the $j$ th diagonal element of the $\mathrm{E}^{+}$Matrix $\left(E_{j j}^{+}\right)$, and evolves on the local reconnection timescale $\left(\tau_{j}\right)$. The twisting mode associated with surface $j$ only has 
finite twisting amplitude at this surface, rotates at the appropriate natural frequency $\left(\omega_{j}^{-}\right)$, has its stability determined by the $j$ th diagonal element of the $E^{-}$Matrix $\left(E_{j j}^{-}\right)$, and also evolves on the local reconnection timescale $\left(\tau_{j}\right)$. Note that twisting parity modes are always stable at iow- $n$, because the diagonal elements of the $\mathrm{E}^{-}$Matrix are always negative.

\section{B. Nonlinear stability}

Suppose that the tearing mode associated with surface 1 is linearly unstable (i.e. $E_{11}^{+}>0$ ), whereas that associated with surface 2 is stable (i.e. $E_{22}^{+}<0$ ). Let $\Psi_{1}^{+}$be the nonlinearly saturated ${ }^{22}$ tearing amplitude at surface 1 , and let $\omega$ be its rotation frequency.

According to Eqs. (17), (22a), (23), and (34)

$$
\begin{aligned}
\frac{\Psi_{1}^{-}}{\Psi_{1}^{+}} & \simeq 0 \\
\frac{\Psi_{2}^{+}}{\Psi_{1}^{+}} & \simeq \frac{E_{12}^{+}}{-\mathrm{i}\left(\omega-\omega_{2}^{+}\right) \tau_{2}-E_{22}^{+}}, \\
\frac{\Psi_{2}^{-}}{\Psi_{1}^{+}} & \simeq \frac{H_{12}}{-\mathrm{i}\left(\omega-\omega_{2}^{-}\right) \tau_{2}-E_{22}^{-}}, \\
\delta T_{\phi, E M}\left(r_{2}\right) & \simeq-\frac{2 n \pi^{2} R_{0}}{\mu_{0}} \times\left[\frac{\left(E_{12}^{+}\right)^{2}\left(\omega-\omega_{2}^{+}\right) \tau_{2}}{\left(\omega-\omega_{2}^{+}\right)^{2} \tau_{2}^{2}+\left(-E_{22}^{+}\right)^{2}}\right. \\
\operatorname{Re}\left(\Delta_{1}^{+}\right) & \left.\simeq E_{11}^{+}+\frac{\left(H_{12}\right)^{2}\left(\omega-\omega_{2}^{-}\right) \tau_{2}}{\left(\omega-\omega_{2}^{-}\right)^{2} \tau_{2}^{2}+\left(-E_{22}^{-}\right)^{2}}\right] \times\left|\Psi_{1}^{+}\right|^{2} \\
\left(\omega-\omega_{2}^{+}\right)^{2} \tau_{2}^{2}+\left(-E_{22}^{+}\right)^{2} & +\frac{\left(H_{12}\right)^{2}\left(-E_{22}^{-}\right)}{\left(\omega-\omega_{2}^{-}\right)^{2} \tau_{2}^{2}+\left(-E_{22}^{-}\right)^{2}}
\end{aligned}
$$

provided $\left|\omega_{1}^{+}-\omega_{1}^{-}\right| \tau_{1} \gg 1$ and $\left|\omega_{2}^{+}-\omega_{2}^{-}\right| \tau_{2} \gg 1$.

As described in Sec. II.D, the nonlinear electromagnetic torques which develop in the plasma modify the bulk toroidal plasma rotation. Note that $\delta T_{\phi \mathrm{EM}}\left(r_{1}\right)=-\delta T_{\phi \mathrm{EM}}\left(r_{2}\right)$, according to Eq. (24). The steady-state shift in the plasma toroidal angular rotation velocity 
is written [see Eq. (25)]

$$
\Omega_{\phi}(r)=\Omega_{\phi}\left(r_{1}\right)\left\{\begin{array}{cl}
1 & r<r_{1} \\
\int_{r}^{r_{2}} \frac{d r}{r \mu_{\perp}(r)} / \int_{r_{1}}^{r_{2}} \frac{d r}{r \mu_{\perp}(r)} & r_{1} \leq r \leq r_{2} \\
& r_{2}<r,
\end{array}\right.
$$

so the viscous restoring torques acting at the two rational surfaces take the form

$$
\delta T_{\phi \mathrm{VS}}\left(r_{2}\right)=-\delta T_{\phi \mathrm{VS}}\left(r_{1}\right)=4 \pi^{2} R_{0}^{3} \times \Omega_{\phi}\left(r_{1}\right) / \int_{r_{1}}^{r_{2}} \frac{d r}{r \mu_{\perp}(r)} .
$$

The changes induced in the toroidal velocity profile Doppler shift the mode rotation frequency, which in the unperturbed plasma is equal to the natural frequency of tearing parity modes at surface 1 , giving

$$
\omega=\omega_{1}^{+}-n \Omega_{\phi}\left(r_{1}\right)
$$

[see (28)]. There is no Doppler shifting of frequencies at surface 2 since $\Omega_{\phi}\left(r_{2}\right)=0$ [see Eq. (38)]. Also, $\omega_{1}^{+}-\omega_{1}^{-}$is unaffected by modifications to the plasma velocity because both frequencies are Doppler shifted by the same amount, so if $\left|\omega_{1}^{+}-\omega_{1}^{-}\right| \tau_{1} \gg 1$ (as is likely to be the case in a high temperature tokamak plasma) then there is always negligible twisting amplitude at surface 1 (see Sec. III).

Torque balance at the rational surfaces [see Eq. (27)] yields

$$
\frac{1}{4} \frac{y^{+}(1-f)}{\left(b^{+}\right)^{2}+(1-f)^{2}}-\frac{1}{4} \frac{y^{-} f}{\left(b^{-}\right)^{2}+f^{2}}=f-f_{1}
$$

where

$$
\begin{aligned}
& f=\frac{\omega-\omega_{2}^{-}}{\omega_{2}^{+}-\omega_{2}^{-}}, \\
& f_{1}=\frac{\omega_{1}^{+}-\omega_{2}^{-}}{\omega_{2}^{+}-\omega_{2}^{-}}, \\
& b^{ \pm}=\frac{-E_{22}^{ \pm}}{\left|\omega_{2}^{+}-\omega_{2}^{-}\right| \tau_{2}},
\end{aligned}
$$




$$
\begin{aligned}
& y^{+}=\frac{\left|E_{12}^{+} \Psi_{1}^{+}\right|^{2}}{\Lambda^{2}}, \\
& y^{-}=\frac{\left|H_{12} \Psi_{1}^{+}\right|^{2}}{\Lambda^{2}}, \\
& \Lambda^{2}=\frac{\mu_{0}}{2} \frac{R_{0}^{2}}{n^{2}}\left(\omega_{2}^{+}-\omega_{2}^{-}\right)^{2} \tau_{2} / \int_{r_{1}}^{r_{2}} \frac{d r}{r \mu_{\perp}(r)} .
\end{aligned}
$$

Here, $f$ is the normalized mode frequency with $f=f_{l}$ in the unperturbed plasma.

In the physically relevant asymptotic limit $b^{ \pm} \ll 1$, Eq. (41) possesses bifurcated solutions. Bifurcations occur in the $y^{+}-y^{-}$plane when the curve of locus

$$
\begin{aligned}
& y^{+}=4\left(2 f-f_{1}\right)(1-f)^{2}, \\
& y^{-}=4\left(1-2 f+f_{1}\right) f^{2}
\end{aligned}
$$

is crossed in the direction of increasing $y^{+}$and $y^{-}$. This critical curve is plotted in Fig. 1 for various values of the unperturbed (normalized) mode frequency $f_{1}$. The solution can either bifurcate to the tearing resonance at surface $2(f=1)$, or the associated twisting resonance $(f=0)$. Prior to bifurcation,

$$
\begin{aligned}
& \omega \neq \omega_{2}^{+} \text {or } \omega_{2}^{-}, \\
& \frac{\Psi_{2}^{ \pm}}{\Psi_{1}^{+}} \simeq 0 \\
& \operatorname{Re}\left(\Delta_{1}^{+}\right) \simeq E_{11}^{+},
\end{aligned}
$$

whereas after bifurcation to the tearing resonance,

$$
\begin{aligned}
& \omega \simeq \omega_{2}^{+}, \\
& \frac{\Psi_{2}^{+}}{\Psi_{1}^{+}} \simeq \frac{E_{12}^{+}}{\left(-E_{22}^{+}\right)}, \\
& \frac{\Psi_{2}^{-}}{\Psi_{1}^{+}} \simeq 0,
\end{aligned}
$$




$$
\operatorname{Re}\left(\Delta_{1}^{+}\right) \simeq E_{11}^{+}+\frac{\left(E_{12}^{+}\right)^{2}}{\left(-E_{22}^{+}\right)}
$$

and after bifurcation to the twisting resonance,

$$
\begin{aligned}
\omega & \simeq \omega_{2}^{-} \\
\frac{\Psi_{2}^{+}}{\Psi_{1}^{+}} & \simeq 0 \\
\frac{\Psi_{2}^{-}}{\Psi_{1}^{+}} & \simeq \frac{H_{12}}{\left(-E_{22}^{-}\right)}, \\
\operatorname{Re}\left(\Delta_{1}^{+}\right) & \simeq E_{11}^{+}+\frac{\left(H_{12}\right)^{2}}{\left(-E_{22}^{-}\right)} .
\end{aligned}
$$

Clearly, bifurcation or 'locking' to the tearing resonance at surface 2 leads to the close coincidence of the mode frequency, $\omega$, and the natural frequency for tearing parity modes, $\omega_{2}^{+}$. After locking, substantial tearing amplitude is driven at surface 2 and the original mode consequently becomes more unstable [i.e. $\Delta_{1}^{+}$increases - see Eq. (45d)]. Likewise, locking to the twisting resonance leads to the close coincidence of $\omega$ and $\omega_{2}^{-}$, with substantial twisting amplitude driven at surface 2 and an associated destabilization of the mode [see Eq. (46d)].

According to Fig. 1 and Eqs. (42), locking occurs to either the tearing or twisting resonance at surface 2 depending on the value of the unperturbed mode frequency $\left(\omega_{1}^{+}\right)$and the relative strengths of the coupling coefficients $E_{12}^{+}$and $H_{12}$. In fact, locking always occurs to the twisting resonance for $\omega_{1}^{+}<\omega_{2}^{-}$, whereas locking always occurs to the tearing resonance for $\omega_{1}^{+}>\omega_{2}^{+}$. Here, it is assumed that $\omega_{2}^{+}-\omega_{2}^{-}>0$ for the sake of definiteness. For $\omega_{2}^{+}>\omega_{1}^{+}>\omega_{2}^{-}$, locking to the twisting resonance takes place provided

$$
\left|\frac{E_{12}^{+}}{H_{12}}\right|^{2}<\frac{\omega_{2}^{+}-\omega_{1}^{+}}{\omega_{1}^{+}-\omega_{2}^{-}}
$$

and locking to the tearing resonance occurs when the converse is true. Of course, locking to either resonance can only take place once the mode amplitude $\left|\Psi_{1}^{+}\right|$has exceeded a critical value of order $\Lambda$ [see Eqs. (42), (43), and Fig. 1]. 
At small, but finite, $b^{ \pm}$it is possible for either the tearing or the twisting resonance at surface 2 to 'disappear' if the associated mode becomes too stable. A resonance effectively ceases to exist once there is no associated zero in the normalized electromagnetic torque [i.e. in the left-hand side of (41)]. This follows because at high mode amplitude (i.e. $y^{+} \gg 1$ and $\left.y^{-} \gg 1\right)$ the system can only 'lock' to a frequency which lies very close to a zero of the normalized torque. It is easily demonstrated that the tearing resonance at surface 2 disappears when

$$
c^{+} \equiv\left|\frac{H_{12}}{E_{12}^{+}}\right|^{2} \frac{2 b^{+}}{1+\left(b^{-}\right)^{2}}>1,
$$

whereas the twisting resonance disappears when

$$
c^{-} \equiv\left|\frac{E_{12}^{+}}{H_{12}}\right|^{2} \frac{2 b^{-}}{1+\left(b^{+}\right)^{2}}>1 \text {. }
$$

Equations (37b), (37c), (42a), and (42c) yield

$$
\begin{aligned}
& \frac{\Psi_{2}^{+}}{\Psi_{1}^{+}} \simeq \frac{E_{12}^{+}}{\left(-E_{22}^{+}\right)} \frac{b^{+}}{-\mathrm{i}(f-1)+b^{+}}, \\
& \frac{\Psi_{2}^{-}}{\Psi_{1}^{+}} \simeq \frac{H_{12}}{\left(-E_{22}^{-}\right)} \frac{b^{-}}{-\mathrm{i} f+b^{-}},
\end{aligned}
$$

so it can be seen by comparison with (45b), (45c), (46b), and (46c) that the linear suppression of driven tearing and twisting amplitude at surface 2 , due to sheared rotation and diamagnetic flows, only occurs when the parameters $b^{+}$and $b^{-}$are much less than unity, respectively.

\section{Conclusions}

The above analysis can be extended to deal with the case of three or more rational surfaces in the plasma in a relatively straightforward manner. In the linear regime the conventional ${ }^{4,7}$ neglect of twisting parity modes in the dispersion relation for low- $n$ modes is justified, since these modes are always stable. However, in the nonlinear regime twisting modes cannot be 
neglected in the dispersion relation, since unstable tearing modes can access additional free energy by coupling to stable twisting modes.

\section{Stability of a Plasma Containing Many Rational Surfaces}

\section{A. Introduction}

Consider the stability of high- $n$ modes for which there are many closely spaced rational surfaces in the plasma. In the vicinity of rational surface $j$, radius $r_{j}$, resonant with poloidal mode number $m_{j}$, the safety factor profile can be expanded

$$
q(r) \simeq q_{j}\left(1+s_{j} x+\cdots\right)
$$

where $x=\left(r-r_{j}\right) / r_{j}, q_{j}=q\left(r_{j}\right)$, and $s_{j}=s\left(r_{j}\right)$. The minor radii and resonant poloidal mode numbers of the neighboring surfaces are then given by

$$
\begin{aligned}
r_{j+k} & \simeq r_{j}\left(1+\frac{k}{m_{j} s_{j}}\right), \\
m_{j+k} & \simeq m_{j}+k
\end{aligned}
$$

\section{B. The $\mathrm{E}^{+}$matrix at high-n}

Consider the behavior of the ideal-MHD equations (A.6) in the high- $n$ limit. Suppose that

$$
\begin{aligned}
m_{j} & \gg 1, \\
\epsilon m_{j} & \gg \kappa_{j},
\end{aligned}
$$


where $\kappa_{j}$ is the current gradient parameter at surface $j$ [see Eq. (15)]. Expansion in the inverse aspect ratio, $\epsilon$, yields

$$
\psi_{m_{j}}^{C}(r)= \begin{cases}\left(\frac{r}{r_{j}}\right)^{m_{j}} & r<r_{j} \\ \left(\frac{r}{r_{j}}\right)^{-m_{j}} & r \geq r_{j}\end{cases}
$$

to lowest order, where the cylindrical tearing basis solution $\psi_{m_{j}}^{C}(r)$ is defined in Sec. A.V. The cylindrical tearing stability index for the $m_{j} / n$ mode takes the form ${ }^{6}$

$$
\Delta_{j}^{0} \simeq-2 m_{j}
$$

so at high- $n$ the diagonal elements of the $\mathrm{E}^{+}$Matrix reduce to

$$
E_{j j}^{+} \simeq-2 m_{j}\left[1+\mathcal{O}\left(\epsilon^{2}\right)\right]
$$

[see Eq. (11)]. Clearly, high- $n$ tearing modes are intrinsically very stable (i.e. $\left.E_{j j}^{+} \ll-1\right)$.

Expansion of the ideal-MHD equations to first order in $\epsilon$ gives the following expression for the off-diagonal elements of the $\mathrm{E}^{+}$Matrix: ${ }^{23,24}$

$$
\begin{aligned}
E_{j j+k}^{+} \simeq \int_{0}^{a}\left[L_{m_{j}}^{m_{j}+k}\right. & \frac{r}{m_{j}} \frac{d \psi_{m_{j}}^{C}}{d r} \frac{r}{m_{j}+k} \frac{d \psi_{m_{j}+k}^{C}}{d r} \\
& -\frac{m_{j}\left(m_{j}+k\right)}{\left(m_{j}+k-n q\right)\left(m_{j}-n q\right)} P_{m_{j}}^{m_{j}+k} \psi_{m_{j}}^{C} \psi_{m_{j}+k}^{C} \\
& +\frac{\left(m_{j}+k\right)}{\left(m_{j}+k-n q\right)} M_{m_{j}}^{m_{j}+k} \frac{r}{m_{j}} \frac{d \psi_{m_{j}}^{C}}{d r} \psi_{m_{j}+k}^{C} \\
& \left.-\frac{m_{j}}{\left(m_{j}-n q\right)} N_{m_{j}}^{m_{j}+k} \frac{r}{m_{j}+k} \frac{d \psi_{m_{j}+k}^{C}}{d r} \psi_{m_{j}}^{C}\right] \frac{d r}{r},
\end{aligned}
$$

where the $\mathcal{O}(\epsilon)$ coupling coefficients $L_{m}^{m+k}(r), P_{m}^{m+k}(r), M_{m}^{m+k}(r)$ and $N_{m}^{m+k}(r)$ are evaluated for a large aspect-ratio, low- $\beta$, weakly shaped tokamak equilibrium in FHMR. ${ }^{4}$ Consider such an equilibrium in which the flux surfaces are specified by

$$
R=R_{0}-r \cos \omega-\Delta(r)+E(r) \cos \omega+T(r) \cos 2 \omega+\mathcal{O}\left(\epsilon^{2} a\right)
$$




$$
Z=r \sin \omega+E^{\prime}(r) \sin \omega+T(r) \sin 2 \omega+\mathcal{O}\left(\epsilon^{2} a\right) .
$$

Here, $(R, \phi, Z)$ are standard cylindrical polar coordinates (with $Z$ in the direction of the toroidal symmetry axis), $R_{0}$ is the plasma major radius, $r$ is a radius-like flux-surface label, $\omega$ is the poloidal angle about the magnetic axis $(r=0), \Delta(r)$ is the Shafranov shift of flux surfaces, $E(r)$ is the flux-surface ellipticity, and $T(r)$ is the flux-surface triangularity. The outermost plasma flux surface lies at $r=a$, where $a$ is the plasma minor radius. The ordering assumptions are that $\epsilon \equiv a / R_{0} \ll 1$, and $\Delta(a), E(a), T(a) \sim \mathcal{O}(\epsilon a)$. After considerable algebra, Eqs. (52), (54), (57), and the expressions for the coupling coefficients contained in FHMR, yield

$$
\begin{aligned}
& E_{j j \pm 1}^{+} \simeq-\frac{2 m_{j}}{s_{j}} \exp \left(-\frac{1}{s_{j}}\right)\left(s_{j} \Delta_{j}^{\prime}+\left[\frac{3}{2}+\frac{1}{2} s_{j}\right] \frac{r_{j}}{R_{0}}\right)+\frac{m_{j} \alpha_{j}}{s_{j}} \exp \left(-\frac{1}{s_{j}}\right) I\left(s_{j}\right), \\
& E_{j j \pm 2}^{+} \simeq \frac{2 m_{j}}{s_{j}} \exp \left(-\frac{2}{s_{j}}\right)\left(\left[1+s_{j}\right] E_{j}^{\prime}+3 \frac{E_{j}}{r_{j}}\right), \\
& E_{j j \pm 3}^{+} \simeq \frac{2 m_{j}}{s_{j}} \exp \left(-\frac{3}{s_{j}}\right)\left(\left[2+s_{j}\right] T_{j}^{\prime}+8 \frac{T_{j}}{r_{j}}\right),
\end{aligned}
$$

where $\Delta_{j}=\Delta\left(r_{j}\right), E_{j}=E\left(r_{j}\right), T_{j}=T\left(r_{j}\right)$, and ' denotes $d / d r$, with $\alpha_{j}$ given by Eq. (13). In the above,

$$
I(s)=(2+s)\left[\gamma+\ln (2 / s) j+s \exp (2 / s) E_{1}(2 / s)-1,\right.
$$

where $\gamma$ is Euler's constant and $E_{1}(x)$ is a standard exponential integral function. ${ }^{25}$ Clearly, the diagonal elements of the $\mathrm{E}^{+}$Matrix are $\mathcal{O}\left(m_{j}\right)$, whereas the off-diagonal elements are $\mathcal{O}\left(\epsilon m_{j}\right)$. Note that $E_{j \neq \pm k}^{+}$is negligible for $k>3$.

\section{The $\mathbf{H}$ matrix at high-n}

The diagonal elements of the H Matrix can be written

$$
H_{j j}=-\frac{\pi}{2} \frac{\kappa_{j}}{s_{j}}+\mathcal{O}\left(\epsilon^{2} m_{j}\right)
$$


according to Eq. (A.40). The off-diagonal elements take the form

$$
H_{j \neq 1} \simeq \mp \frac{\pi}{2} \frac{m_{j} \alpha_{j}}{s_{j}}\left(2+s_{j}\right) \exp \left(-\frac{1}{s_{j}}\right),
$$

where use has been made of Eqs. (52), (54), and (A.48). Thus, the diagonal elements of the $\mathrm{H}$ Matrix are $\mathcal{O}(1)$, whereas the off-diagonal elements are $\mathcal{O}\left(\epsilon m_{j}\right)$. Note that $H_{j} j \pm k$ is negligible for $k>1$.

\section{The $\mathrm{E}^{-}$matrix at high-n}

At high- $n$ the cylindrical basis solutions $\psi_{m, \pm 1}^{L}(r)$ and $\psi_{m, \pm 1}^{R}(r)$, defined in Sec. A.VI, can be built up out of a linear combination of $r^{m_{j}}$ and $r^{-m}$. It is easily demonstrated that

$$
\begin{aligned}
& \Delta_{m_{j}+1}^{L}=-\Delta_{m_{j}-1}^{R} \simeq m_{j}, \\
& \Delta_{m_{j}-1}^{L}=-\Delta_{m_{j}+1}^{R} \simeq m_{j} \frac{1+\exp \left(-2 / s_{j}\right)}{1-\exp \left(-2 / s_{j}\right)},
\end{aligned}
$$

where use has been made of Eqs. (52) and (A.53). Thus, according to Eqs. (22b) and (A.56) the diagonal elements of the $\mathrm{E}^{-}$Matrix take the form

$$
E_{j j}^{-} \simeq-\Delta_{j}^{c}+\frac{\pi^{2}}{4} \frac{m_{j} \alpha_{j}^{2}}{s_{j}}\left(2+s_{j}\right)\left[1-\frac{\left(2+s_{j}\right)}{s_{j}} \exp \left(-\frac{2}{s_{j}}\right)\right] .
$$

Clearly, high- $n$ twisting modes can become intrinsically unstable (i.e. $E_{j j}^{-}>0$ ) provided $\epsilon^{2} m_{j} \sim \mathcal{O}(1)$. Note that $E_{j \neq k}^{-}$is negligible for $k>0$.

\section{E. The dispersion relation at high- $n$}

The dispersion relation for coupled tearing and twisting modes at surface $j$ is written [see Eq. (10)]:

$$
\begin{aligned}
& \Delta_{j}^{+}(\omega) \Psi_{j}^{+}=\sum_{k}\left(E_{j k}^{+} \Psi_{k}^{+}+H_{j k} \Psi_{k}^{-}\right), \\
& \Delta_{j}^{-}(\omega) \Psi_{j}^{-}=\sum_{k}\left(E_{j k}^{-} \Psi_{k}^{-}+H_{k j} \Psi_{k}^{+}\right) .
\end{aligned}
$$


Let

$$
\begin{aligned}
\epsilon m_{j} & \gg 1 \\
\epsilon^{2} m_{j} & \sim \mathcal{O}(1),
\end{aligned}
$$

which is consistent with Eqs. (53) and also allows twisting modes to become intrinsically unstable. It follows from Secs. V.B to V.D that

$$
\begin{aligned}
& E_{j j}^{+} \sim \mathcal{O}\left(m_{j}\right), \quad E_{j j \pm k}^{+} \sim \mathcal{O}\left(\epsilon m_{j}\right) \\
& H_{j j} \sim \mathcal{O}(1), \quad H_{j j \pm 1} \sim \mathcal{O}\left(\epsilon m_{j}\right) \\
& E_{j j}^{-} \sim \mathcal{O}(1)
\end{aligned}
$$

for $1 \leq k \leq 3$, with all other matrix elements negligible in the adopted ordering scheme. Suppose that

$$
\Psi_{k}^{+} \lesssim \mathcal{O}(\epsilon) \Psi_{j}^{-}
$$

for all $k$ [see Eqs. (87)]. The dispersion relation at surface $j$ then reduces to

$$
\begin{aligned}
& \Delta_{j}^{+}(\omega) \Psi_{j}^{+}=E_{j j}^{+} \Psi_{j}^{+}+H_{j j+1} \Psi_{j+1}^{-}+H_{j j-1} \Psi_{j-1}^{-}+\mathcal{O}\left(\Psi_{j}^{-}\right) \\
& \Delta_{j}^{-}(\omega) \Psi_{j}^{-}=E_{j j}^{-} \Psi_{j}^{-}+H_{j+1} \Psi_{j+1}^{+}+H_{j-1} \Psi_{j-1}^{+}+\mathcal{O}\left(\epsilon \Psi_{j}^{-}\right)
\end{aligned}
$$

with the aid of Eqs. (66) and (67). Thus, at high- $n$ the direct coupling between tearing modes (i.e. the off-diagonal elements of the $\mathrm{E}^{+}$Matrix) can be neglected with respect to the coupling between tearing and twisting modes (i.e. the off-diagonal elements of the $\mathrm{H}$ Matrix). The dominant coupling is that between tearing and twisting modes whose poloidal mode numbers differ by unity. According to Eqs. (23) and (69), the electromagnetic torque acting at surface $j$ is given by 


$$
\begin{array}{r}
\delta T_{\phi \mathrm{EM}}\left(r_{j}\right) \simeq \frac{2 n \pi^{2} R_{0}}{\mu_{0}} \times \operatorname{Im}\left[H_{j j+1} \Psi_{j+1}^{-}\left(\Psi_{j}^{+}\right)^{*}+H_{j j-1} \Psi_{j-1}^{-}\left(\Psi_{j}^{+}\right)^{*}\right. \\
\left.\quad+H_{j+1 j} \Psi_{j+1}^{+}\left(\Psi_{j}^{-}\right)^{*}+H_{j-1 j} \Psi_{j-1}^{+}\left(\Psi_{j}^{-}\right)^{*}\right]
\end{array}
$$

Equations (69) govern the behavior of tearing and twisting modes at a general rational surface $j$. An analogous set of equations can be written for all of the neighboring rational surfaces, giving rise to a net dispersion relation consisting of a very large number of coupled equations. However, this dispersion relation can be greatly simplified by noting from Eq. (52a) that at high- $n$ the coupled rational surfaces are very closely spaced. Suppose that the surfaces are sufficiently close together that there is very little variation in equilibrium parameters from surface to surface. Then,

$$
\begin{aligned}
E_{j j}^{+} & \simeq E_{0}^{+}(r)=-2 n q \\
H_{j j-1} & \simeq-H_{j j+1} \simeq H_{1}(r)=\frac{\pi}{2} \frac{n q \alpha}{s}(2+s) \exp \left(-\frac{1}{s}\right), \\
E_{j j}^{-} & \simeq E_{0}^{-}(r)=-\Delta^{c}(r)+\frac{\pi^{2}}{4} \frac{n q \alpha^{2}}{s}(2+s)\left[1-\frac{(2+s)}{s} \exp \left(-\frac{2}{s}\right)\right]
\end{aligned}
$$

for rational surfaces in the vicinity of minor radius $r$, where use has been made of Eqs. (56), (62), and (64). Here, $q(r)$ is the safety factor, $s(r)=r q^{\prime} / q$ the magnetic shear, $\alpha(r)=$ $-\left(2 R_{0} \mu_{0} p^{\prime} q^{2} / B_{0}^{2}\right)$ the pressure gradient parameter, and $\Delta^{c}(r) \equiv \Delta_{j}^{c}\left(r_{j} \rightarrow r\right)$ [see Eq. (20)] a measure of the stabilizing effect of plasma compressibility on twisting modes. Let

$$
\begin{gathered}
\Psi_{j}^{ \pm}=-\Psi_{j \pm 2}^{ \pm}=\Psi_{j \pm 4}^{ \pm} \cdots=\Psi_{0}^{ \pm}(r), \\
\Psi_{j+1}^{ \pm}=-\Psi_{j+1 \pm 2}^{ \pm}=\Psi_{j+1 \pm 4}^{ \pm} \cdots=\Psi_{1}^{ \pm}(r)
\end{gathered}
$$

and

$$
\delta T_{\phi \mathrm{EM}}\left(r_{j}\right)=-\delta T_{\phi \mathrm{EM}}\left(r_{j \pm 1}\right)=\delta T_{\phi \mathrm{EM}}\left(r_{j \pm 2}\right) \cdots=T_{0}(r)
$$


with

$$
\begin{gathered}
\Delta_{j}^{ \pm}(\omega)=\Delta_{j \pm 2}^{ \pm}(\omega) \cdots=\Delta_{0}^{ \pm}(\omega), \\
\Delta_{j+1}^{ \pm}(\omega)=\Delta_{j+1 \pm 2}^{ \pm}(\omega) \cdots=\Delta_{1}^{ \pm}(\omega),
\end{gathered}
$$

then Eqs. (69) and (70) yield

$$
\begin{aligned}
& \left\{\Delta_{0}^{+}(\omega)-E_{0}^{+}\right\} \Psi_{0}^{+}=-2 H_{1} \Psi_{1}^{-}, \\
& \left\{\Delta_{0}^{-}(\omega)-E_{0}^{-}\right\} \Psi_{0}^{-}=2 H_{1} \Psi_{1}^{+} \\
& \left\{\Delta_{1}^{+}(\omega)-E_{0}^{+}\right\} \Psi_{1}^{+}=2 H_{1} \Psi_{0}^{-} \\
& \left\{\Delta_{1}^{-}(\omega)-E_{0}^{-}\right\} \Psi_{1}^{-}=-2 H_{1} \Psi_{0}^{+}
\end{aligned}
$$

and

$$
T_{0}=\frac{2 n \pi^{2} R_{0}}{\mu_{0}} \times 2 H_{1} \times \operatorname{Im}\left[\Psi_{1}^{+}\left(\Psi_{0}^{-}\right)^{*}+\Psi_{0}^{+}\left(\Psi_{1}^{-}\right)^{*}\right] .
$$

Thus, according to Eqs. (72), at high- $n$ coupled twisting and tearing modes form a nonlocalized periodic structure which repeats every fourth rational surface. According to (73), equal and opposite electromagnetic torques act on alternate rational surfaces. These torques modify the plasma toroidal velocity profile. Let

$$
\Omega_{\phi}\left(r_{j}\right)=-\Omega_{\phi}\left(r_{j \pm 1}\right)=\Omega_{\phi}\left(r_{j \pm 2}\right) \cdots=\frac{1}{2} \Omega_{0}(r)
$$

where $\Omega_{\phi}\left(r_{k}\right)$ is the change in the plasma toroidal angular velocity at surface $k$, then balancing viscous and electromagnetic torques in the plasma gives

$$
4 \pi^{2} R_{0} \times \mu_{\perp} R_{0}^{2} \times 2 n q s \Omega_{n}=T_{0}
$$

where $\mu_{\perp}(r)$ is the plasma perpendicular viscosity, and use has been made of Eqs. (25)-(27), (52a), and (73). 


\section{F. Linear stability at high- $n$}

The analysis of the linear stability of Eqs. (75) in a plasma possessing sheared rotation and diamagnetic flows yields decoupled tearing and twisting modes whose stability is determined by $E_{0}^{+}$and $E_{0}^{-}$, respectively (see Sec. IV). The tearing modes are found to be very stable, since $E_{0}^{+} \ll-1$ at high- $n$ [see Eq. (71a)], whereas the twisting modes become unstable for $n$ sufficiently large to ensure that $E_{0}^{-}>0$ [see Eq. (71c)].

\section{G. Nonlinear stability at high-n}

Consider the nonlinear stability of linearly unstable twisting modes. The changes induced in the toroidal velocity profile by the electromagnetic torques Doppler shift the natural frequencies of twisting modes so that modes on alternate rational surfaces rotate differentially. Thus, modes on 'even' surfaces (i.e. $j, j \pm 2$, etc.) rotate at

$$
\omega=\omega^{-}(r)-\frac{n}{2} \Omega_{0}(r)
$$

whereas those on 'odd' surfaces (i.e. $j \pm 1, j \pm 3$, etc.) rotate at

$$
\omega=\omega^{-}(r)+\frac{n}{2} \Omega_{0}(r)
$$

[see Eqs. (28) and (77)]. In the above, $\omega^{-}(r) \equiv \omega_{j}^{-}\left(r_{j} \rightarrow r\right)$ is the natural frequency of twisting parity modes resonant close to minor radius $r$ in the unperturbed plasma. The modifications to the plasma toroidal velocity also Doppler shift the natural frequencies of tearing modes, which become $\omega^{+}(r)-(n / 2) \Omega_{0}(r)$ at even surfaces and $\omega^{+}(r)+(n / 2) \Omega_{0}(r)$ at odd surfaces. Here, $\omega^{+}(r) \equiv \omega_{j}^{+}\left(r_{j} \rightarrow r\right)$ is the natural frequency of tearing parity modes resonant close to radius $r$ in the unperturbed plasma.

According to Eq. (75a), the twisting amplitude at odd rational surfaces drives co-rotating tearing amplitude at even surfaces, so that

$$
\frac{\Psi_{0}^{+}}{\Psi_{1}^{-}}=\frac{-2 H_{1}}{-\mathrm{i}\left(\omega^{-}-\omega^{+}+n \Omega_{0}\right) \tau+\left(-E_{0}^{+}\right)},
$$


where use has been made of (17) and (80). In the above, $\tau(r) \equiv \tau_{j}\left(r_{r} \rightarrow r\right)$ is the reconnection timescale for rational surfaces located close to minor radius $r$. Likewise, according to Eq. (75c), the twisting amplitude at even surfaces drives co-rotating tearing amplitude at odd surfaces, with

$$
\frac{\Psi_{1}^{+}}{\Psi_{0}^{-}}=\frac{2 H_{1}}{-\mathrm{i}\left(\omega^{-}-\omega^{+}-n \Omega_{0}\right) \tau+\left(-E_{0}^{+}\right)} .
$$

It follows from (76), (81), and (82) that

$$
\begin{aligned}
T_{0}= & \frac{2 n \pi^{2} R_{0}}{\mu_{0}} \times 4\left(H_{1}\right)^{2} \times \\
& {\left[\frac{\left(\omega^{-}-\omega^{+}-n \Omega_{0}\right) \tau\left|\Psi_{0}^{-}\right|^{2}}{\left(\omega^{-}-\omega^{+}-n \Omega_{0}\right)^{2} \tau^{2}+\left(-E_{0}^{+}\right)^{2}}-\frac{\left(\omega^{-}-\omega^{+}+n \Omega_{0}\right) \tau\left|\Psi_{1}^{-}\right|^{2}}{\left(\omega^{-}-\omega^{+}+n \Omega_{\jmath}\right)^{2} \tau^{2}+\left(-E_{0}^{+}\right)^{2}}\right], }
\end{aligned}
$$

Finally, the stability of twisting modes at even and odd surfaces is governed by

$$
\operatorname{Re}\left(\Delta_{0}^{-}\right)=E_{0}^{-}+\frac{4\left(H_{1}\right)^{2}\left(-E_{0}^{+}\right)}{\left(\omega^{-}-\omega^{+}-n \Omega_{0}\right)^{2} \tau^{2}+\left(-E_{0}^{+}\right)^{2}},
$$

and

$$
\operatorname{Re}\left(\Delta_{1}^{-}\right)=E_{0}^{-}+\frac{4\left(H_{1}\right)^{2}\left(-E_{0}^{+}\right)}{\left(\omega^{-}-\omega^{+}+n \Omega_{0}\right)^{2} \tau^{2}+\left(-E_{0}^{+}\right)^{2}},
$$

respectively [see Eqs. (75b) and (75d)].

Let

$$
\begin{aligned}
f(r) & =\frac{n \Omega_{0}}{\omega^{-}-\omega^{+}}, \\
b(r) & =\frac{2 n q}{\left(\omega^{-}-\omega^{+}\right) \tau}, \\
\Lambda^{2}(r) & =\frac{2 \mu_{0}}{n \pi^{2}} \times \frac{s^{3}}{q(2+s)^{2}} \exp \left(\frac{2}{s}\right) \times \mu_{\perp} R_{0}^{2} \times \frac{\left(\omega^{-}-\omega^{+}\right)^{2} \tau}{(n \alpha)^{2}} \\
y_{0,1}(r) & =\frac{\left|\Psi_{0,1}^{-}\right|^{2}}{\Lambda^{2}}
\end{aligned}
$$

then according to Eqs. (81) and (82) the tearing amplitudes driven at the even and orld 
rational surfaces are given by

$$
\begin{aligned}
& \frac{\Psi_{0}^{+}}{\Psi_{1}^{-}}=\frac{\pi}{2} \frac{\alpha}{s}(2+s) \exp \left(-\frac{1}{s}\right) \frac{b}{-\mathrm{i}(1+f)+b}, \\
& \frac{\Psi_{1}^{+}}{\Psi_{0}^{-}}=\frac{\pi}{2} \frac{\alpha}{s}(2+s) \exp \left(-\frac{1}{s}\right) \frac{b}{-\mathrm{i}(1-f)+b} .
\end{aligned}
$$

Note that the above equations are consistent with the ordering assumption (68). In addition, Eqs. (84) and (85) yield

$$
\begin{aligned}
& \operatorname{Re}\left(\Delta_{0}^{-}\right)=-\Delta^{c}+\frac{\pi^{2}}{4} \frac{n q \alpha^{2}}{s}(2+s)\left[1+\frac{b^{2}-(1-f)^{2}}{b^{2}+(1-f)^{2}} \frac{(2+s)}{s} \exp \left(-\frac{2}{s}\right)\right] \\
& \operatorname{Re}\left(\Delta_{1}^{-}\right)=-\Delta^{c}+\frac{\pi^{2}}{4} \frac{n q \alpha^{2}}{s}(2+s)\left[1+\frac{b^{2}-(1+f)^{2}}{b^{2}+(1+f)^{2}} \frac{(2+s)}{s} \exp \left(-\frac{2}{s}\right)\right]
\end{aligned}
$$

where use has been made of (71). Finally, the torque balance equation (78) reduces to

$$
\frac{1}{2} \frac{y_{0}(1-f)}{b^{2}+(1-f)^{2}}-\frac{1}{2} \frac{y_{1}(1+f)}{b^{2}+(1+f)^{2}}=f,
$$

with the aid of Eq. (83).

According to Eqs. (87), twisting modes at the even and odd rational surfaces share the stability index

$$
\operatorname{Re}\left(\Delta_{0}^{-}\right) \simeq \operatorname{Re}\left(\Delta_{1}^{-}\right) \simeq-\Delta^{c}+\frac{\pi^{2}}{4} \frac{n q \alpha^{2}}{s}(2+s)\left[1-\frac{1-b^{2}}{1+b^{2}} \frac{(2+s)}{s} \exp \left(-\frac{2}{s}\right)\right]
$$

in the linear regime (i.e. $f=0$ ). This suggests that the twisting fluxes at both sets of surfaces have the same initial amplitudes. Suppose that

$$
\Psi_{0}^{-} \simeq \Psi_{1}^{-} \simeq \Psi^{-}
$$

and

$$
y_{0} \simeq y_{1} \simeq y \equiv \frac{\left|\Psi^{-}\right|^{2}}{\Lambda^{2}}
$$

Now, the left-hand side of Eq. (89) represents the normalized electromagnetic torque, $t_{\mathrm{EM}}(f)$, acting at rational surfaces, whereas the right-hand side represents the viscous restoring 
torque, $t_{\mathrm{vs}}(f)$. The roots of (89) are equivalent to the stationary states of an imaginary particle located at coordinate $f$ and moving in the potential

$$
V(f)=\int_{0}^{f}\left(t_{\mathrm{VS}}-t_{\mathrm{EM}}\right) d f .
$$

Thus, stable stationary states are characterized by $V^{\prime}(f)=0$ and $V^{\prime \prime}(f)<0$. Equations (89), (92), and (J3) yield

$$
V(f)=\frac{1}{2} f^{2}+\frac{y}{2} \ln \sqrt{b^{2}+(1+f)^{2}}+\frac{y}{2} \ln \sqrt{b^{2}+(1-f)^{2}} .
$$

It is easily demonstrated that $f=0$ is always a stationary state of the potential (94), but is only stable for

$$
y<y_{c}=\frac{\left(1+b^{2}\right)^{2}}{\left(1-b^{2}\right)} .
$$

For $b<\sqrt{2}-1$, the solution bifurcates to either

$$
f=+\sqrt{\frac{\left(1+2 b-b^{2}\right)\left(1-2 b-b^{2}\right)}{\left(1-b^{2}\right)}},
$$

or

$$
f=-\sqrt{\frac{\left(1+2 b-b^{2}\right)\left(1-2 b-b^{2}\right)}{\left(1-b^{2}\right)}},
$$

at $y=y_{c}$. Both states are equally likely, since $V(f)$ is even in $f$. For $1>b>\sqrt{2}-1$, there is no jump in $f$ at the bifurcation point. Instead, the solution connects smoothly to either an $f>0$ or an $f<0$ branch at $y=y_{c}$. Note from Eq. (95) that $y_{c} \rightarrow \infty$ as $b \rightarrow 1$. In fact, for $b \geq 1$ there are no bifurcations, and $f=0$ remains a stable stationary state of (94) for all values of $y$.

\section{H. Discussion}

The nonlinear behavior of high- $n$ twisting modes is governed by the parameter $b$, defined in Eq. (86b). 
Consider the limit $b \ll 1$, for which the difference in natural frequencies of tearing and twisting modes is sufficiently large to suppress driven magnetic reconnection in the unperturbed state. In fact, it can be seen from Eqs. (87) and (90) that in the unperturbed state $(f=0)$ there is very little tearing amplitude driven at rational surfaces, and the twisting mode stability index consequently reduces to

$$
\operatorname{Re}\left(\Delta_{0}^{-}\right) \simeq \operatorname{Re}\left(\Delta_{1}^{-}\right) \simeq-\Delta^{c}+\frac{\pi^{2}}{4} \frac{n q \alpha^{2}}{s}(2+s)\left[1-\frac{(2+s)}{s} \exp \left(-\frac{2}{s}\right)\right] .
$$

The system remains in the unperturbed state as long as the magnitude of the twisting amplitude $\left|\Psi^{-}\right|$lies below the critical value $\Lambda$, defined in Eq. (86c). As the critical amplitude is exceeded there is a sudden change in the plasma toroidal angular velocity profile such as to bring the twisting frequency at one set of rational surfaces (either the 'even' or the 'odd' surfaces defined in the previous section) into close coincidence with the tearing frequency of the other set (i.e. $f \simeq 1$ or $f \simeq-1$ ). This process is (rather loosely) termed 'locking.' After locking, substantial tearing amplitude is driven at one set of surfaces - the even surfaces, say - so that according to Eqs. (87)

$$
\frac{\Psi_{0}^{+}}{\Psi_{1}^{-}} \simeq \frac{\pi}{2} \frac{\alpha}{s}(2+s) \exp \left(-\frac{1}{s}\right)
$$

while there is still very little driven tearing flux at the odd surfaces. The stability index for twisting modes at the odd surfaces becomes

$$
\operatorname{Re}\left(\Delta_{1}^{-}\right) \simeq-\Delta^{c}+\frac{\pi^{2}}{4} \frac{n q \alpha^{2}}{s}(2+s)\left[1+\frac{(2+s)}{s} \exp \left(-\frac{2}{s}\right)\right]
$$

after locking, whereas the stability index at the even surfaces is unchanged [see Eqs. (88) and (98)].

Thus, above a certain critical twisting amplitude there is a transition from an initial state which is symmetric with respect to the even and odd rational surfaces, to a final state which is highly asymmetric. There are no driven magnetic islands in the initial state, whereas the 
final state is characterized by magnetic islands on alternate rational surfaces. According to Eqs. (72) and (99), the driven islands form an interlocking structure in which the O-points of a given island chain line up with the X-points of the neighboring chains. This structure repeats every fourth rational surface. The initial and final states are sketched in Fig. 2. In the initial state twisting modes on all rational surfaces have the same stability index [see Eq. (98)]. However, in the final state twisting modes on the rational surfaces with no driven magnetic islands become significantly more unstable than those on surfaces with islands [compare Eqs. (98) and (100)].

Consider the limit $b \gg 1$, for which the differences in the natural frequencies of tearing and twisting modes are too smali to suppress driven magnetic reconnection. In this limit, there is no modification to the plasma toroidal velocity profile. According to Eqs. (87) and (88), substantial tearing amplitude is driven on both sets of rational surfaces, and twisting modes on all surfaces have the stability index (100). If the mutual coupling between tearing modes, which was previously neglected, is taken into account it is found that the islands on odd surfaces lock in phase quadrature with respect to those on even surfaces, giving rise to a symmetric configuration in which there is a. $\pi / 2$ phase shift between island chains on neighboring surfaces. This state, which is sketched in Fig. 3 , is that predicted by conventional ballooning mode theory. ${ }^{26}$ The $\pi / 2$ phase shift between island chains corresponds to $\theta_{0}=\pi / 2$, where $\theta_{0}$ is the ballooning phase angle, and Eq. (100) is equivalent to

$$
\Delta_{B}^{\prime} \simeq \frac{\pi}{4} \frac{\alpha^{2}}{s^{2}}(2+s)\left[1+\frac{(2+s)}{s} \exp \left(-\frac{2}{s}\right)\right],
$$

where $\Delta_{B}^{\prime}$ is the ballooning stability index. It is clear that ballooning mode analysis is only valid in the limit where there is no substantial difference between the natural frequencies of tearing and twisting parity modes (i.e. $b \gg 1$ ).

Note that the mismatch between the twisting and tearing frequencies, $\omega^{-}-\omega^{+}$, scales like a diamagnetic frequency (i.e. like $n$ ). It follows from (86b) that the parameter $b$ is 
approximately independent of mode number (there is, in fact, some weak dependence of the reconnection timescale $\tau$ on $n$ ). This implies that $b \ll 1$ is the physically relevant ordering for high- $n$ modes, just as it is for low- $n$ modes (see Secs. III and IV). It can be seen from Eq. (86c) that the threshold amplitude scales like $n^{-1 / 2}$. So at sufficiently high mode numbers there are bound to be magnetic islands driven in the plasma by unstable twisting modes. It follows from Eq. (99) that when the threshold amplitude is exceeded the driven tearing amplitude is $\mathcal{O}(\epsilon)$ with respect to the twisting amplitude.

\section{Conclusions}

In the linear regime the conventional neglect of tearing parity modes in the high- $n$ dispersion relation ${ }^{3}$ is justified because high- $n$ tearing modes are very stable and are effectively decoupled from high- $n$ twisting modes via diamagnetic flows. However, in the nonlinear regime the neglect of tearing parity modes is not justified. It has been demonstrated that in a plasma possessing diamagnetic flows there is a threshold amplitude for twisting modes below which there are no driven high- $n$ magnetic islands. As the threshiold is exceeded, however, substantial magnetic islands are driven on every second rational surface, giving

rise to an interlocking structure which repeats every fourth surface. Conventional ballooning analysis ${ }^{26}$ is found to give a poor description of coupled high- $n$ tearing and twisting modes in situations where diamagnetic flows are important.

\section{Implications for Ohmically Heated Tokamaks}

The aim of this section is to make some quantitative predictions regarding the stability of low- $n$ coupled tearing and twisting modes in ohmically heated tokamaks.

Consider the stability of $n=1$ modes in a family of idealized tokamaks of constant aspect ratio, $a=0.35 R_{0}$, with a toroidal magnetic field strength which scales like $B_{0}(\mathrm{~T})=$ $1.38 R_{0}^{0.7}(\mathrm{~m})$. Broadly speaking, most modern tokamaks of conventional design are members 
of this family; for instance, CoMPASS-C $\left(R_{0}=0.56 \mathrm{~m}, a=0.20 \mathrm{~m}, B_{0}=1.1 \mathrm{~T}\right),{ }^{27}$ DIII-D $\left(R_{0}=1.67 \mathrm{~m}, a=0.64 \mathrm{~m}, B_{0}=1.3 \mathrm{~T}\right),{ }^{28}$ JET $\left(R_{0}=3.0 \mathrm{~m}, a=1.1 \mathrm{~m}, B_{0}=3.0 \mathrm{~T}\right),{ }^{1}$ ITER (1991) $\left(R_{0} \sim 6.0 \mathrm{~m}, a \sim 2.2 \mathrm{~m}, B_{0} \sim 4.9 \mathrm{~T}\right),{ }^{29}$ and ITER $(1993)\left(R_{0} \sim 8.0 \mathrm{~m}\right.$, $\left.a \sim 2.8 \mathrm{~m}, B_{0} \sim 6.0 \mathrm{~T}\right) .^{30}$ Plausible temperature and electron density profiles are adopted [i.e. $T(r) \propto\left(1-r^{2} / a^{2}\right)^{3 / 2}$ and $n_{e}(r) \propto \sqrt{1-r^{2} / a^{2}}$, which inply a parabolic pressure profile $\left.p(r) \propto\left(1-r^{2} / a^{2}\right)^{2}\right]$. The central electron temperature is estimated from ohmic power balance, using the standard neo-Alcator energy confinement timescale. ${ }^{31}$ No provision is made for the neoclassical enhancement of resistivity or the shaping of plasma cross-sections, but this is offset to some extent by adopting an artificially high value of $Z_{\text {eff }}$ (i.e. $Z_{\text {eff }}=$ 4). The central ion temperature is estimated by balancing the volume averaged rate of heating by the electrons, calculated using the classical energy exchange timescale, against the volume averaged rate of energy losses, calculated using the neo-Alcator energy confinement timescale. The viscosity profile is assumed to be flat (for want of a better assumption), and the anomalous momentum confinement timescale (i.e. the exponential decay timescale of an unsupported velocity profile) is set equal to the neo-Alcator energy confinement timescale. ${ }^{19}$ The discharges investigated have deuterium as the fueling ion species and a line averaged electron number density $\bar{n}_{e}=2 \times 10^{19} \mathrm{~m}^{-3}$. The adopted safety factor profile is that described in FHMR, ${ }^{4}$ which reduces to

$$
q(r)=q_{a} \frac{r^{2} / a^{2}}{1-\left(1-r^{2} / a^{2}\right)^{q_{a} / q_{0}}}
$$

in the cylindrical limit, where $q_{0}$ is the safety factor on the magnetic axis $(r=0)$, and $q_{a}$ is the safety factor at the plasma edge $(r=a)$. In the following calculations the central $q$ value is fixed at $q_{0}=0.7$. Table I shows basic plasma parameters, including the central electron and ion temperatures and the central beta $\left[\beta_{c}=\mu_{0} p(0) / B_{0}^{2}\right]$, estimated as a function of major radius, using the method outlined above, for discharges with $q_{a}=4.5$.

Consider the interaction of the unstable $m=2$ tearing mode with the stable $m=3$ 


\begin{tabular}{|ccc|ccc|}
\hline$R_{0}(\mathrm{~m})$ & $a(\mathrm{~m})$ & $B_{0}(\mathrm{~T})$ & $T_{e c}(\mathrm{keV})$ & $T_{i c}(\mathrm{keV})$ & $\beta_{c}$ \\
\hline 0.50 & 0.18 & 0.85 & 0.55 & 0.35 & $6.41 \times 10^{-3}$ \\
0.75 & 0.26 & 1.13 & 0.82 & 0.64 & $5.84 \times 10^{-3}$ \\
1.00 & 0.35 & 1.38 & 1.09 & 0.92 & $5.40 \times 10^{-3}$ \\
1.50 & 0.53 & 1.83 & 1.62 & 1.48 & $4.74 \times 10^{-3}$ \\
2.00 & 0.70 & 2.24 & 2.15 & 2.03 & $4.27 \times 10^{-3}$ \\
3.00 & 1.05 & 2.98 & 3.21 & 3.11 & $3.65 \times 10^{-3}$ \\
4.00 & 1.40 & 3.64 & 4.26 & 4.17 & $3.26 \times 10^{-3}$ \\
6.00 & 2.10 & 4.84 & 6.35 & 6.29 & $2.77 \times 10^{-3}$ \\
8.00 & 2.80 & 5.92 & 8.43 & 8.37 & $2.44 \times 10^{-3}$ \\
\hline
\end{tabular}

Table I: Basic plasma parameters estimated for ohmically heated tokamaks with $a=$ $0.35 R_{0}, B_{0}(\mathrm{~T})=1.38 R_{0}^{0.7}(\mathrm{~m}), q_{0}=0.7, q_{a}=4.5$, and $\bar{n}_{e}=2 \times 10^{19} \mathrm{~m}^{-3}$, including the central electron temperature $\left(T_{e c}\right)$, the central ion temperature $\left(T_{i c}\right)$, and the central plasma beta $\left(\beta_{c}\right)$.

tearing and twisting modes. The former mode is resonant at the $q=2$ surface (radius $r_{2}=0.658 a$, with magnetic shear $s_{2}=1.74$ ), whereas the latter modes are resonant at $q=3$ $\left(r_{3}=0.816 a, s_{3}=1.98\right)$. The presence of a $q=1$ surface in the plasma $\left(r_{1}=0.366 a\right.$, $\left.s_{1}=0.694\right)$ strongly morlifies the stability of the $2 / 1$ tearing mode due to coupling with the $1 / 1$ internal kink mode, but does not unduly affect either the stability of the modes resonant at $q=3$ or the coupling of these modes to the $2 / 1$ tearing mode, provided that the plasma cross-section is circular (as is assumed to be the case). ${ }^{14}$ Table II shows various interesting plasma parameters evaluated at the $q=3$ surface using data from Table I. The chosen parameters are the local hydromagnetic, resistive, and viscous timescales $\left(\tau_{H}, \tau_{R}\right.$, and $\tau_{V}$, respectively). the reconnection timescale $\left[\tau_{3}\right.$, calculated in accordance with (19)], the local beta $\left[\beta_{3}\right.$, calculated in accordance with Sec. II.C, assuming $\left.\gamma_{s}=5 / 3\right]$, the critical twisting mode stability index needed to overcome the stabilizing effect of plasma compressibility $\left[\Delta_{3}^{c}\right.$, calculated in accordance with (20)], the local electron diamagnetic frequency $\left(\omega_{3}^{*}\right)$, the critical frequency (relative to the natural frequency) above which compressibility is negligible in the tearing and twisting mode dispersion relations $\left[\omega_{3}^{c} \equiv V_{c} \tau_{H}-\right.$ see Eq. (B.32b)], and the critical frequencies $\omega_{3}^{(1)}$ and $\omega_{3}^{(2)}$ defined in Eqs. (21a) and (21b). 


\begin{tabular}{|c|c|c|c|c|c|c|c|c|}
\hline$R_{0}$ & $\tau_{H}$ & $\tau_{R}$ & $\tau_{3}$ & $\Delta_{3}^{c}$ & $\omega_{3}^{*}$ & $\omega_{3}^{c}$ & $\omega_{3}^{(1)}$ & $\omega_{3}^{(2)}$ \\
\hline 0.50 & 0.73 & $39.21 \times 10^{0} 1.06 \times 10^{1}$ & $3.78 \times 10^{-1}$ & $1.19 \times 10^{-3} 3.47 \times 10^{0}$ & $2.33 \times 10^{1}$ & $1.46 \times 10^{0}$ & $3.95 \times 10^{1}$ & $4.54 \times 10^{1}$ \\
\hline 75 & 0. & $33.70 \times 10^{2} 3.59 \times 10^{1}$ & $1.02 \times 10^{0}$ & $1.09 \times 10^{-3} 5.35 \times 10^{0}$ & $1.16 \times 10^{1}$ & $8.33 \times 10^{-1}$ & $2.56 \times 10^{1}$ & $2.49 \times 10^{1}$ \\
\hline .00 & 0.90 & $9.84 \times 10^{1} 8.46 \times 10^{1}$ & $2.06 \times 10^{0}$ & $1.00 \times 10^{-3} 7.22 \times 10^{0}$ & $7.11 \times 10^{0}$ & $5.57 \times 10^{-1}$ & $.90 \times 10^{1}$ & $1.63 \times 10^{1}$ \\
\hline 50 & 1.02 & $23.91 \times 10^{2} 2.86 \times 10^{2}$ & $5.54 \times 10^{0}$ & $8.82 \times 10^{-4} 1.08 \times 10^{1}$ & $3.55 \times 10^{0}$ & $3.11 \times 10^{-1}$ & $1.23 \times 10^{1}$ & $8.98 \times 10^{0}$ \\
\hline 00 & 1.11 & $11.05 \times 10^{3} 6.79 \times 10^{2}$ & $1.12 \times 10^{1}$ & $7.94 \times 10^{-4} 1.44 \times 10^{1}$ & $2.17 \times 10^{0}$ & $2.05 \times 10^{-1}$ & $10^{\circ}$ & $10^{\circ}$ \\
\hline 00 & 1.26 & $64.18 \times 10^{3} 2.29 \times 10^{3}$ & $3.02 \times 10^{1}$ & $6.79 \times 10^{-4} 2.16 \times 10^{1}$ & $1.08 \times 10^{0}$ & $1.13 \times 10^{-1}$ & $5.88 \times 10^{0}$ & $3.22 \times 10^{0}$ \\
\hline 4.00 & 1.37 & $71.11 \times 10^{4} 5.43 \times 10^{3}$ & $6.10 \times 10^{1}$ & $6.06 \times 10^{-4} 2.85 \times 10^{1}$ & $6.59 \times 10^{-1}$ & $7.44 \times 10^{-2}$ & $4.33 \times 10^{0}$ & $2.11 \times 10^{0}$ \\
\hline$\infty$ & 1.5 & $54.47 \times 10^{4} 1.83 \times 10^{4}$ & $1.65 \times 10^{2}$ & $5.15 \times 10^{-4} 4.25 \times 10^{1}$ & $3.29 \times 10^{-1}$ & $10^{-}$ & $10^{0}$ & $1.16 \times 10^{0}$ \\
\hline 00 & 1.68 & $81.19 \times 10^{5} 4.35 \times 10^{4}$ & $3.33 \times 10^{2}$ & $4.54 \times 10^{-4} 5.59 \times 10^{1}$ & $2.00 \times 10^{-1}$ & $2.67 \times 10^{-2}$ & $2.08 \times 10^{0}$ & $7.56 \times 10^{-1}$ \\
\hline
\end{tabular}

Table II: Various plasma parameters evaluated at the $q=3$ surface as a function of major radius $R_{0}$ (in meters) using data from Table $I$. The parameters are the hydromagnetic, resistive, viscous, and reconnection timescales $\left(\tau_{H}\right.$ is in units of $10^{-7}$ seconds, the other timescales are in milliseconds), the local beta, and the critical twisting stability index needed to over come the stabilizing effect of plasma compressibility, the local electron diamagnetic frequency, and three other critical frequencies described in the text. All frequencies are evaluated in kilo-Hertz.

It can be seen that the critical twisting stability index needed to overcome compressibility is modest in small, relatively cold tokamaks, but becomes quite high in large, relatively hot tokamaks. The difference between the natural frequencies of the tearing and twisting modes resonant at $q=3$ is taken to be of order the local electron diamagnetic frequency, $\omega_{3}^{*}$ [see Sec. B.III], and this is also assumed to be the typical frequency mismatch between the $q=2$ and $q=3$ surfaces due to velocity shear and diamagnetic flows. It follows that since $\omega_{3}^{*} \ll \omega_{3}^{(1)}, \omega_{3}^{(2)}$, the inequalities (21a) and (21b) are likely to be satisfied in an ohmically heated tokamak plasma. The third inequality (21c) is also easily satisfied according to Table II. This implies that the visco-resistive dispersion relations (17) and (18), for tearing and twisting modes, respectively, are the most physically relevant in ohmic discharges. ${ }^{19}$ Note that $\omega_{3}^{*} \gg \omega_{3}^{c}$, so compressibility is only important in tearing and twisting dispersion relations in a relatively narrow band of frequencies centered on the natural frequency. This justifies the absence of the compressional Pfirsch-Schlüter enhancement of inertia ${ }^{16}$ (by $1+2 q^{2}$ ) in the layer dispersion relations (see Sec. B.II.2).

Table III shows various critical parameters associated with the locking of the $2 / 1$ tearing 
mode to the tearing and twisting modes resonant at $q=3$, evaluated as a function of major radius using daia from Table II. The poloidal beta $\left(\beta_{p}\right)$ is defined in FHMR. The elements of the $\mathrm{E}^{+}$Matrix are calculated as an expansion in the inverse aspect ratio and poloidal beta using the $\mathrm{T} 7$ code. ${ }^{4}$ For a free boundary plasma

$$
\begin{aligned}
& E_{33}^{+}=-4.631-25.26 \epsilon^{2}-3.454 \epsilon^{2} \beta_{p}^{2}-29.73 \epsilon^{2} \beta_{p}, \\
& E_{23}^{+}=-6.295 \epsilon+4.757 \times 10^{-2} \epsilon \beta_{p} .
\end{aligned}
$$

The elements of the $\mathrm{E}^{-}$Matrix and the $\mathrm{H}$ Matrix are evaluated with the aid of a modified cylindrical tearing mode code using the method outlined in Appendix A and Sec. II.B. For a free boundary plasma

$$
\begin{aligned}
& E_{33}^{-}=-\Delta_{3}^{c}+1.115 \beta_{p}^{2}, \\
& H_{23}=-1.843 \beta_{p} .
\end{aligned}
$$

It can be seen from Table III that $E_{33}^{+}$, which governs the intrinsic stability of the $3 / 1$ tearing mode, is much less than -1 , indicating that this mode is fairly stable. $E_{33}^{+}$also has relatively little variation with major radius. On the other hand, $E_{33}^{-}$, which governs the intrinsic stability of the $3 / 1$ twisting mode, varies strongly with major radius because of its dependence on the layer quantity $\Delta_{3}^{c}$, which scales as $S^{2 / 3}\left(S=\tau_{R} / \tau_{H}\right.$ is the magnetic Fleynolds number). Thus, in small, relatively cold devices the $3 / 1$ twisting mode is moderately stable, whereas in large, relatively hot devices the mode becomes extremely stable. The matrix element $E_{23}^{+}$, which governs the coupling between the $2 / 1$ and $3 / 1$ tearing modes, has virtually no variation with major radius due to its very weak dependence on pressure. On the other hand, the element $H_{23}$, which governs the coupling of the $2 / 1$ tearing mode to the $3 / 1$ twisting mode, has a strong variation with major radius due to its $\beta_{p}$ dependence.

The parameters $b^{+}$and $b^{-}$, defined in Eq. (42c), govern the nature of locking to the $3 / 1$ tearing and twisting modes, respectively. (Note that surfaces 1 and 2 in Sec. IV.B are 


\begin{tabular}{|c|c|cccc|cc|cc|cc|c|c|}
\hline$R_{0}$ & $\beta_{p}$ & $-E_{33}^{+}$ & $-E_{33}^{-}$ & $-E_{23}^{+}-H_{23}$ & $b^{+}$ & $b^{-}$ & $c^{+}$ & $c^{-}$ & $D^{+}$ & $D^{-}$ & $\lambda_{c}$ & $\widehat{\Lambda}$ \\
\hline 0.50 & 0.71 & 10.51 & 2.91 & 2.23 & 1.30 & 0.190 & 0.053 & $2.2 \times 10^{-1}$ & 0.30 & 0.473 & 0.581 & 0.25 & $4.99 \times 10^{-3}$ \\
0.75 & 0.64 & 10.24 & 4.89 & 2.23 & 1.19 & 0.138 & 0.066 & $7.8 \times 10^{-2}$ & 0.46 & 0.486 & 0.290 & 0.22 & $2.51 \times 10^{-3}$ \\
1.00 & 0.60 & 10.04 & 6.82 & 2.23 & 1.10 & 0.109 & 0.074 & $5.3 \times 10^{-2}$ & 0.60 & 0.495 & 0.177 & 0.20 & $1.55 \times 10^{-3}$ \\
1.50 & 0.52 & 9.74 & 10.50 & 2.23 & 0.96 & 0.079 & 0.085 & $2.9 \times 10^{-2}$ & 0.91 & 0.511 & 0.088 & 0.16 & $7.80 \times 10^{-4}$ \\
2.00 & 0.47 & 9.53 & 14.20 & 2.23 & 0.87 & 0.062 & 0.093 & $1.9 \times 10^{-2}$ & 1.22 & 0.522 & - & - & $4.79 \times 10^{-4}$ \\
3.00 & 0.40 & 9.26 & 21.42 & 2.23 & 0.74 & 0.045 & 0.105 & $9.9 \times 10^{-3}$ & 1.81 & 0.537 & - & - & $2.42 \times 10^{-4}$ \\
4.00 & 0.36 & 9.09 & 28.36 & 2.23 & 0.66 & 0.036 & 0.112 & $6.3 \times 10^{-3}$ & 2.53 & 0.547 & - & - & $1.48 \times 10^{-4}$ \\
6.00 & 0.31 & 8.88 & 42.40 & 2.23 & 0.56 & 0.026 & 0.124 & $3.2 \times 10^{-3}$ & 3.92 & 0.560 & - & - & $7.49 \times 10^{-5}$ \\
8.00 & 0.27 & 8.74 & 55.82 & 2.23 & 0.50 & 0.021 & 0.133 & $2.0 \times 10^{-3}$ & 5.37 & 0.568 & - & - & $4.55 \times 10^{-5}$ \\
\hline
\end{tabular}

Table III: Critical parameters governing the locking of the $2 / 1$ tearing mode to the tearing and twisting modes resonant at $q=3$ evaluated as a function of the major radius $R_{0}$ (in meters) using data from Table II. The various parameters are described in the text.

equivalent to surfaces 2 and 3 here.) In Table III these parameters are calculated assuming that $\omega_{3}^{+}-\omega_{3}^{-}=\omega_{3}^{*}$, as discussed previously. According to Sec. IV.B, if $b^{+} \ll 1$ there is very little driven tearing amplitude at $q=3$ prior to locking [see Eq. (50a)]. However, as the locking threshold is exceeded, there is a sudden bifurcation to a state where the driven tearing amplitude is given by Eq. (45b). As $b^{+}$is increased, the transition from the initial to the final state becomes gradually less sudden, until there is eventually no bifurcation, and there is substantial tearing amplitude driven at $q=3$ even in the initial state. In the absence of the twisting resonance, the bifurcation disappears for $b^{+}>1 / \sqrt{27}=0.1925 .{ }^{4}$ Locking to the twisting resonance at $q=3$ is, likewise, governed by the parameter $b^{-}$. It can be seen from Table III that $b^{+}$decreases with increasing major radius, indicating an increasingly sharp locking transition to the $3 / 1$ tearing resonance. Conversely, the parameter $b^{-}$increases with $R_{0}$, indicating an increasingly smooth locking transition to the $3 / 1$ twisting resonance. Both $b^{+}$and $b^{-}$are sufficiently small to ensure that bifurcations occur during locking to the tearing and twisting resonances at $q=3$.

The tearing resonance at $q=3$ disappears when the parameter $c^{+}$, defined in Eq. (48), becomes greater than unity. Likewise, the twisting resonance disappears when $c^{-}>1$, where $c^{-}$is defined in Eq. (49). Table III indicates that the tearing resonance is present 
at all major radii, whereas the twisting resonance is only present in small, relatively cold tokamak plasmas.

In the limit $b^{+} \ll 1$, locking to the $3 / 1$ resonance gives rise to an increase in the stability index for the $2 / 1$ tearing mode by $D^{+} \equiv\left(E_{23}^{+}\right)^{2} /\left(-E_{33}^{+}\right)$[see Eq. (45d)]. Likewise, in the limit $b^{-} \ll 1$, locking to the $3 / 1$ twisting resonance gives rise to an increase in the $2 / 1$ tearing mode stability index by $D^{-} \equiv\left(H_{23}\right)^{2} /\left(-E_{33}^{-}\right)$[see Eq. (46d)]. Table III indicates that locking to the $3 / 1$ tearing mode gives rise to a modest further destabilization of the $2 / 1$ tearing mode at all major radii. In very small devices, locking to the $3 / 1$ twisting mode also gives rise to modest destabilization of the $2 / 1$ mode, but this effect attenuates rapidly with increasing $R_{0}$.

The $2 / 1$ tearing mode locks to either the $3 / 1$ tearing resonance or the $3 / 1$ twisting resonance, depending on its initial rotation frequency, $\omega_{2}^{+}$. For $\omega_{2}^{+}<\omega_{3}^{-}+\lambda_{c}\left(\omega_{3}^{+}-\omega_{3}^{-}\right)$ the locking is to the twisting resonance (assuming $\omega_{3}^{+}>\omega_{3}^{-}$). Otherwise, the locking is to the tearing resonance. Here, $\lambda_{c}=\left(1+\left|E_{23}^{+}\right|^{2} /\left|H_{23}\right|^{2}\right)^{-1}$ [see Eq. (47)]. It can be seen from Table III that as the major radius increases, locking to the tearing resonance becomes more likely. For $R_{0} \gtrsim 2.0 \mathrm{~m}$, the twisting resonance disappears, so locking to the tearing resonance occurs at all values of $\omega_{2}^{+}$in this regime.

According to Eqs. (42) and (43), the critical 2/1 tearing amplitude for locking to the tearing resonance at $q=3$ is of order $\Lambda /\left|E_{23}^{+}\right|$, whereas the critical amplitude for locking to the twisting resonance is approximately $\Lambda /\left|H_{23}\right|$. Table III shows values of the dimensionless quantity $\hat{\Lambda} \equiv \Lambda /\left(a B_{0}\right)$ evaluated as a function of major radius. It can be seen that the critical $2 / 1$ tearing amplitude needed to lock to the $q=3$ resonances decreases rapidly with increasing machine dimensions. ${ }^{4}$

The data shown in Table III is calculated for $q_{a}=4.5$. It is found that increasing the edge- $q$ tends to favor locking to the twisting resonance, since the local beta at $q=3$ rises, leading to an increase in the coupling coefficient $H_{23}$. Conversely, decreasing the edge- $q$ 
tends to favor locking to the tearing resonance.

\section{Summary and Conclusions}

\section{A. The form of the dispersion relation}

A dispersion relation has been derived for resistive modes of arbitrary parity in a tokamak plasma [see Eqs. (10)]. In a plasma containing $N$ rational surfaces (resonant with a given toroidal mode number $n$ ) there are, in general, $2 N$ independent resistive modes. It is convenient to resolve a general mode into components of $N$ basis tearing modes and $N$ basis twisting modes. The $j$ th basis tearing mode $(1 \leq j \leq N)$ is defined to have unit tearing amplitude and zero twisting amplitude at rational surface $j$ (rational surfaces are numbered in order of increasing minor radius), with zero tearing or twisting amplitude at any other surface. Likewise, the $j$ th basis twisting mode has unit twisting amplitude and zero tearing amplitude at surface $j$, with zero tearing or twisting amplitude at any other surface. Here, the tearing amplitude at surface $j$ is basically the even (with respect to the rational surface) component of the perturbed normal resonant magnetic field, whereas the twisting amplitude is the odd component (see Appendix A for more exact definitions).

The intrinsic stability and mutual interactions of the $N$ basis tearing modes are specified by a real symmetric $N \times N$ matrix known as the $\mathrm{E}^{+}$Matrix (see Sec. II.B). The elements of this matrix can, in general, only be evaluated by solving the full coupled ideal-MHD equations in the outer region. This can be achieved for a large aspect-ratio, low- $\beta$, weakly shaped tokamak equilibrium using the recently developed T7 code, as described in FHMR. ${ }^{4}$ In a plasma with a monotonic safety factor profile containing no rational surfaces resonant with poloidal mode number $m=1$, the $j$ th basis tearing mode is made up of $\mathcal{O}(1)$ of the poloidal harmonic resonant at rational surface $j$ (poloidal mode number $m_{j}$, say), with $\mathcal{O}(\epsilon)$ side-band poloidal harmonics (i.e. $m_{j} \pm 1$ coupled by toroidicity and pressure, $m_{j} \pm 2$ coupled by the ellipticity of equilibrium flux surfaces, and $m_{j} \pm 3$ coupled by flux surface triangularity). 
Here, the inverse aspect-ratio, $\epsilon \ll 1$, is the ratio of the minor and major radii of the plasma. The $j$ th diagonal element of the $\mathrm{E}^{+}$Matrix, which governs the intrinsic stability of the $j$ th basis tearing mode, is made up of the standard cylindrical tearing stability index for the $m_{j} / n \operatorname{mode}^{8}$ plus a $\mathcal{O}\left(\epsilon^{2}\right)$ correction. The off-diagonal elements of the $\mathrm{E}^{+}$Matrix, which govern interaction between different basis tearing modes, are $\mathcal{O}(\epsilon)$. At high poloidal mode number $\left(m_{j} \gg 1\right)$, the $j$ th basis tearing mode becomes localized in the vicinity of rational surface $j$, but the side-band harmonics remain $\mathcal{O}(\epsilon)$ with respect to the resonant harmonic. In this limit, the $j$ th diagonal element of the $\mathrm{E}^{+}$Matrix asymptotes to $-2 m_{j}$ (i.e. the $j$ th basis tearing mode becomes very stable), whereas the off-diagonal elements become $\mathcal{O}\left(\epsilon m_{j}\right)$. See Secs. II.B and V.B for more details.

The intrinsic stability and mutual interactions of the $N$ basis twisting modes are specified by another real symmetric $N \times N$ matrix known as the $\mathrm{E}^{-}$Matrix (see Sec. II.B). In a low- $\beta$ plasma the ordering $\nu \sim \mathcal{O}\left(\epsilon^{2}\right)$, where $\nu$ is the Mercier index related to the well-known Mercier stability criterion $\frac{1}{4}-\nu>0,{ }^{11}$ can be exploited to greatly simplify the calculation of this matrix, as described in $\mathrm{CHT}^{6}$ and Appendix A. In a plasma with a monotonic safety factor profile, the $j$ th basis twisting mode is made up of $\mathcal{O}(1)$ of the $m_{j}$ poloidal harmonic, localized inside the resonant layer at surface $j$, with $\mathcal{O}(\alpha)$ of the $m_{j} \pm 1$ side-band harmonics, and only $\mathcal{O}\left(\alpha^{2}\right)$ of the $m_{j}$ harmonic, exterior to the layer. Here, $\alpha$ is a $\mathcal{O}(\epsilon)$ parameter proportional to the local pressure gradient at rational surface $j$. The $j$ th diagonal element of the $\mathrm{E}^{-}$Matrix, which governs the intrinsic stability of the $j$ th basis twisting mode, is made up of a stabilizing term emanating from the layer at surface $j$ plus an $\mathcal{O}\left(\alpha^{2}\right)$ destabilizing correction. The layer term is due to the effect of plasma compressibility, and scales like $\beta^{1 / 2} S^{2 / 3}$ (where $\beta$ is the usual ratio of plasma and magnetic pressures, and $S$ is the magnetic Reynolds number). The off-diagonal elements of the $\mathrm{E}^{-}$Matrix are zero, indicating that there is no direct interaction between different basis twisting modes. At high poloidal mode number, the $j$ th basis twisting mode becomes localized in the vicinity of rational surface $j$, 
but the relative magnitudes of the various poloidal harmonics inside and outside the layer remain the same. In this limit, the destabilizing correction to the $j$ th diagonal element of the $E^{-}$Matrix is $\mathcal{O}\left(\alpha^{2} m_{j}\right)$, so the $j$ th basis twisting mode can become intrinsically unstable when $\alpha^{2} m_{j} \gtrsim \mathcal{O}(1)$. See Secs. II.B, II.C, and V.D for more details.

It is clear from the above discussion that the $j$ th basis tearing mode is the generalization of the $m_{j} / n$ cylindrical tearing mode in toroidal geometry, whereas the $j$ th basis twisting mode is the toroidal generalization of the $m_{j} / n$ resistive interchange mode.

The interaction of the $N$ basis tearing modes with the $N$ basis twisting modes is governed by a real $N \times N$ matrix known as the $\mathrm{H}$ Matrix (see Sec. II.B). In a low- $\beta$ plasma the ordering $\nu \sim \mathcal{O}\left(\epsilon^{2}\right)$ can again be exploited to greatly simplify the calculation of this matrix (see Appendix A). In a plasma with a monotonic safety factor profile the $\mathrm{H}$ Matrix is tridiagonal. The $j$ th diagonal element, which governs the interaction of the $j$ th basis tearing and twisting modes, is $\mathcal{O}(1)$ and proportional to the local current gradient at rational surface $j$. The off-diagonal elements, which govern the interaction of basis tearing and twisting modes with resonant poloidal mode numbers differing by unity, are $\mathcal{O}(\alpha)$. At high poloidal mode number, the diagonal elements remain $\mathcal{O}(1)$ but the off-diagonal elements become $\mathcal{O}\left(\alpha m_{j}\right)$. See Secs. II.B and V.C for more details.

The responses of the resistive layers at the $N$ rational surfaces in the plasma to tearing and twisting parity perturbations from the outer region are specified by the diagonal matrices $\Delta^{+}$and $\Delta^{-}$. (The $j$ th diagonal element of $\Delta^{+}$specifies the response of the $j$ th layer to a tearing parity perturbation, and the $j$ th diagonal element of $\Delta^{-}$specifies the response to a twisting parity perturbation.) It turns out that the responses of resistive layers to external perturbations are resonant in nature. ${ }^{15,32}$ That is, there is virtually no tearing or twisting amplitude driven in a layer unless the external tearing or twisting parity perturbation rotates in a certain very narrow band of frequencies. The optimum frequency for externally driven tearing amplitude at surface $j$ is equal to the 'natural frequency' of the $j$ th basis tearing 
mode (i.e. the propagation frequency of the uncoupled, intrinsically unstable $j$ th basis tearing mode). Likewise, the optimum frequency for externally driven twisting amplitude at surface $j$ is equal to the natural frequency of the $j$ th basis twisting mode. Typically, the natural frequencies of the $j$ th basis tearing and twisting modes differ by of order the local electron diamagnetic frequency at surface $j$. In addition, sheared rotation and diamagnetic flows in the plasma ensure that the natural frequencies of basis modes associated with different rational surfaces do not match up.

In Appendix B the response of a resistive layer to an external perturbation is investigated in detail, and the model layer dispersion relations (17) and (22a) are derived. In addition, an expression for the stabilizing contribution to the diagonal elements of the $\mathrm{E}^{-}$Matrix, due to plasma compressibility, is obtained [see Eq. (20)]. Appendix B is largely concerned with the effects of anomalous plasma viscosity and plasma compressibility. However, many other effects are neglected for the sake of clarity. Among the important effects which are left out of the analysis are finite ion-Larmor radius, field-line curvature, ${ }^{16}$ diamagnetism $^{33}$ (excepting the different propagation frequencies of tearing and twisting modes), and trapped particle destabilization. ${ }^{18}$ The neglect of these effects is justified, to some extent, because none of them modify the resonant nature of the layer response, which is the crucial factor governing the interaction of the various resistive modes in the plasma.

\section{B. Low- $n$ stability}

Low- $n$ stability is investigated in Secs. III, IV, and VI of this paper. It is found that the differing natural frequencies of basis tearing and twisting modes, due to sheared rotation and diamagnetic flows, lead to the effective decoupling of the $2 N$ basis modes at low amplitude. Now, at low- $n$, the basis twisting modes are all intrinsically stable due to the dominant stabilizing effect of plasma compressibility. So, at low amplitude only the intrinsically unstable basis tearing modes are of practical interest. 
At finite amplitude, an intrinsically unstable basis tearing mode exerts nonlinear electromagnetic torques at the various rational surfaces in the plasma which act so as to bring the different natural frequencies of basis tearing and twisting modes closer together. This process eventually allows modes to develop which have finite tearing or twisting amplitude simultaneously at more than one rational surface in the plasma. These 'compound' modes are always found to be more unstable than the basis modes from which they are constructed. In a conventional tokamak plasma this process takes place in a highly discontinuous manner. Below a certain threshold in mode amplitude, there is some bringing together of the various natural frequencies, but they still remain sufficiently disparate to decouple the basis modes. However, as the threshold amplitude is exceeded, there is a discontinuous change in the plasma toroidal rotation profile, leading to the 'locking' together of the natural frequencies of basis modes associated with two or more rational surfaces in the plasma. This allows the formation of compound modes.

An intrinsically unstable basis tearing mode can 'lock' the natural frequency of either the tearing or the twisting basis mode associated with a given rational surface. However, it is unable to lock both frequencies simultaneously, because they are generally substantially different. It follows that after locking the perturbed magnetic field in the immediate vicinity of the surface possesses either pure tearing or pure twisting parity (i.e. there is either substantial driven tearing amplitude, or substantial driven twisting amplitude, at the surface, but not both at the same time). The locking of a surface in tearing parity leads to the formation of a symmetric chain of magnetic islands whose width is proportional to the square root of the mode amplitude ${ }^{34}$ Locking a surface in twisting parity leads to the formation of a much narrower chain of 'skewed' magnetic islands whose width is directly proportional to the mode amplitude. Thus, locking a surface in twisting parity is likely to cause less degradation of the plasma confinement than that caused by locking it in tearing parity.

Section VI examines the implications of the above results for ohmically heated tokamaks. 
It is found that in large, relatively low- $\beta$ [see Table I] devices, compound modes are likely to have tearing parity at all of the coupled rational surfaces, whereas in small, relatively high- $\beta$ devices, compound modes may have twisting parity at some (but not all) of the coupled surfaces. Compound modes with some twisting parity surfaces are more likely to occur in auxiliary heated plasmas, which generally have higher $\beta$ values than ohmic plasmas.

\section{High- $n$ stability}

High- $n$ stability is investigated in Sec. V. At large toroidal mode number, basis tearing modes become highly stable, whereas basis twisting modes can become unstable for $n \alpha^{2} \gtrsim \mathcal{O}(1)$. The dominant coupling is that between basis tearing and twisting modes whose resonant poloidal mode numbers differ by unity. This coupling is mediated by the off-diagonal elements of the $\mathrm{H}$ Matrix, which are $\mathcal{O}(n \alpha)$. Other couplings, for example those between different basis tearing modes, or those between basis tearing and twisting modes of the same resonant poloidal mode number, are negligible in the high- $n$ limit.

In Sec. $\mathrm{V}$ the close spacing of coupled rational surfaces at high- $n$ is exploited to simplify the analysis. A dispersion relation is constructed for a periodic mode which repeats after every fourth rational surface [see Eqs. (75)]. The mode structure also repeats every second surface with a $180^{\circ}$ phase shift. The structure is, in general, significantly different at neighboring surfaces. This type of mode is a generalization of the conventional low- $\beta$ resistive ballooning mode, whose structure repeats at every surface with a $90^{\circ}$ phase shift. ${ }^{26}$ In fact, the conventional ballooning mode is only obtained in the analysis of Sec. V when the difference in natural frequencies of basis tearing and twisting modes is negligible.

At low amplitude, the generalized mode has twisting parity at all of the coupled rational surfaces, and is significantly more stable than a conventional ballooning mode. However, as a certain critical mode amplitude is exceeded, there is a bifurcation to a new mode structure which has significant driven tearing amplitude at every alternate surface. After 
the bifurcation, the stability of the mode becomes the same as that of a ballooning mode, but its structure is still significantly different. The driven tearing amplitude leads to the formation of (Rutherford type) island chains at every alternate surface. The driven islands form an interlocking structure in which the O-points on a given chain line up with the $\mathrm{X}$ points of neighboring chains (see Fig. 2). This type of structure is likely to severely degrade the plasma confinement.

\section{Conclusions}

It is clear that the conventional approach of neglecting twisting parity modes at low wavelength ${ }^{7}$ [i.e. $n \sim \mathcal{O}(1)$ ] is only appropriate in extremely low- $\beta$ plasmas. ${ }^{13}$ In fact, many ohmically heated plasmas are not sufficiently low- $\beta$ for this approximation to be valid, and it is almost certainly invalid in auxiliary heated plasmas. The conventional approach of neglecting tearing parity modes at short wavelength ${ }^{3}$ [i.e $\left.n \gg \mathcal{O}(1)\right]$ is never appropriate, although under certain circumstances this approach yields the correct twisting mode stability index.

\section{Acknowledgments}

The author would like to thank J.W. Connor, R.J. Hastie, and T.C. Hender of Culham Laboratory (UK) for many illuminating discussions.

This work was jointly funded by the UK Department of Trade and Industry, Euratom, and the U.S. Department of Energy under contract \# DE-FG05-80ET-53088. 


\section{A Physics of the Outer Region}

\section{The marginally-stable ideal-MHD equations}

The coordinate system $(r, \theta, \phi)$, where $\phi$ is the toroidal angle, $\theta$ is an angle-like variable in the poloidal plane, and $r$ is a flux-surface label with dimensions of length, is chosen so that the magnetic field lines appear straight. The Jacobian for these coordinates is given by ${ }^{35}$

$$
j=(\nabla r \wedge \nabla \theta \cdot \nabla \phi)^{-1}=\frac{r R^{2}}{R_{0}},
$$

where $R$ is the major radius, and $R_{0}$ is the average major radius of the outermost plasma flux surface. For an axisymmetric equilibrium the magnetic field $\mathbf{B}$ can be written

$$
\mathbf{B}=B_{0} R_{0}[f(r) \nabla \phi \wedge \nabla r+g(r) \nabla \phi]
$$

where $B_{0}$ is the vacuum magnetic field strength at $R=R_{0}$. The safety factor, the slope of the field lines in the $\theta-\phi$ plane, is then given by

$$
q(r)=\frac{r g(r)}{R_{0} f(r)} .
$$

The perturbed magnetic field $\delta \mathbf{B}$ is completely specified by two sets of fux-surface functions, $\psi_{m}(r)$ and $Z_{m}(r)$, where

$$
\begin{aligned}
\delta \mathbf{B} \cdot \nabla r & =\mathrm{i} \frac{R_{0}^{2}}{R^{2}} \sum_{m} \frac{\psi_{m}(r)}{r} \exp [\mathrm{i}(m \theta-n \phi)], \\
R_{0} \delta \mathbf{B} \cdot \nabla \phi & =n \frac{R_{0}^{2}}{R^{2}} \sum_{m} \frac{\left[Z_{m}(r)+\lambda_{m} \psi_{m}(r)\right]}{R_{0}(m-n q)} \exp [\mathrm{i}(m \theta-n \phi)],
\end{aligned}
$$

with

$$
\lambda_{m}=\left[m \frac{\mu_{0} r p^{\prime}}{B_{0}^{2} f^{2}}\left\langle\frac{R^{2}}{R_{0}^{2}} \frac{1}{|\nabla r|^{2}}\right\rangle-\frac{m}{n}(m-n q) \frac{R_{0} g^{\prime}}{f}\left\langle\frac{1}{|\nabla r|^{2}}\right\rangle\right] / L_{m}^{m}
$$




$$
\begin{aligned}
L_{m}^{m} & =\left[m^{2}\left\langle\frac{1}{|\nabla r|^{2}}\right\rangle+n^{2} \frac{r^{2}}{R_{0}^{2}}\right], \\
\langle\cdots\rangle & \equiv \frac{1}{2 \pi} \oint(\cdots) d \theta .
\end{aligned}
$$

Here, ' denotes differentiation with respect to $r$, and $p(r)$ is the plasma pressure.

Throughout the bulk of the plasma the perturbed field is governed by the marginallystable equations of ideal magnetohydrodynamics (MHD), which take the form:

$$
\begin{array}{r}
r \frac{d \psi_{m}}{d r}=\frac{L_{m}^{m} Z_{m}}{(m-n q)}+\sum_{k \neq 0} \frac{\left(L_{m}^{m+k} Z_{m+k}+M_{m}^{m+k} \psi_{m+k}\right)}{(m+k-n q)}, \\
(m-n q) r \frac{d}{d r}\left[\frac{Z_{m}}{(m-n q)}\right]=\frac{P_{m}^{m} \psi_{m}}{(m-n q)}+\sum_{k \neq 0} \frac{\left(N_{m}^{m+k} Z_{m+k}+P_{m}^{m+k} \psi_{m+k}\right)}{(m+k-n q)} .
\end{array}
$$

The coefficients $L_{m}^{m+k}, M_{m}^{m+k}, N_{m}^{m+k}$ and $P_{m}^{m+k}$ are evaluated for a general low- $\beta$, large aspect-ratio tokamak equilibrium in FHMR. The ordering adopted is such that the Shafranov shift and departure from circularity of plasma flux surfaces are both $\mathcal{O}(\epsilon)$ with respect to the average minor radius of the outermost plasma flux surface, $a$, where $\epsilon=a / R_{0} \ll 1$ is the inverse aspect-ratio. This implies that $L_{m}^{m} \sim \mathcal{O}(1)+\mathcal{O}\left(\epsilon^{2}\right)$ and $L_{m}^{m+k} \sim \mathcal{O}(\epsilon)$, with a similar ordering for the other coefficients. Coupling of harmonics of the perturbed field whose poloidal mode numbers differ by unity is effected by the Shafranov shift of flux surfaces, which is driven by toroidicity and the plasma pressure. Coupling of harmonics whose mode numbers differ by two or three is effected by flux-surface ellipticity or triangularity, respectively. The ordering adopted for the Shafranov shift and flux-surface shaping implies that $g=1+\mathcal{O}\left(\epsilon^{2}\right)$ and $\mu_{0} p / B_{0}^{2} \sim \mathcal{O}\left(\epsilon^{2}\right)$.

\section{The outer solution in the vicinity of a rational surface}

The marginally-stable ideal-MHD equations (A.6) become singular on flux surfaces where the safety factor $q$ takes the rational value $m / n$. Such surfaces are termed rational surfaces resonant with poloidal mode number $m$. The most general solution of Eqs. (A.6) in the 
vicinity of a rational surface, radius $r_{m}$, is written: ${ }^{4}$

$$
\begin{gathered}
\psi_{m} \simeq A^{L, R}|x|^{\nu}[1+\lambda x]+B^{L, R}|x|^{1-\nu}+C x+\cdots, \\
L_{0} Z_{m} \simeq A^{L, R}|x|^{\nu}[\nu+\gamma x]+B^{L, R}(1-\nu)|x|^{1-\nu}+D x+\cdots, \\
\psi_{m+k} \simeq A^{L, R} a_{k}|x|^{\nu}+B^{L, R} \tilde{a}_{k}|x|^{1-\nu}+\bar{\psi}_{m+k}+\cdots, \\
Z_{m+k} \simeq A^{L, R} b_{k}|x|^{\nu}+B^{L, R} \tilde{b}_{k}|x|^{1-\nu}+\bar{Z}_{m+k}+\cdots,
\end{gathered}
$$

where $x=\left(r-r_{m}\right) / r_{m}, L_{0}=-\left(L_{m}^{m}\right)_{r_{m}} / m s$, and

$$
\nu \simeq-\frac{\left(P_{m}^{m}\right)_{r_{m}}}{s^{2}}=\left[-\frac{2 \mu_{0} r p^{\prime}}{B_{0}^{2} s^{2}}\left(1-q^{2}\right)\right]_{r_{m}}
$$

represents the effect of average field-line curvature. ${ }^{11}$ Here, $s=\left(r q^{\prime} / q\right)_{r_{m}}$ is the local magnetic shear; $A^{L, R}=A^{L}$ for $x<0$ and $A^{R}$ for $x>0$, where $A^{L}$ and $A^{R}$ are arbitrary constants, and similarly for $B^{L, R}$. The quantities $\bar{\psi}_{m+k}$ and $\bar{Z}_{m+k}$ are also arbitrary constants. Furthermore,

$$
\begin{aligned}
& \lambda \simeq \frac{1}{2}\left[\frac{\kappa}{s \nu}+1+\left(\frac{r q^{\prime \prime}}{q^{\prime}}\right)_{r_{m}}\right]-\frac{1}{m s \nu} \sum_{k \neq 0} \frac{1}{k}\left(L_{m}^{m+k} P_{m}^{m+k}-M_{m}^{m+k} N_{m}^{m+k}\right)_{r_{m}}, \\
& \gamma=\frac{1}{2}\left[\frac{\kappa}{s \nu}(1+\nu)+1+\left(\frac{r q^{\prime \prime}}{q^{\prime}}\right)_{r_{m}}\right]-\frac{1}{m s} \sum_{k \neq 0} \frac{1}{k}\left(L_{m}^{m+k} P_{m}^{m+k}-M_{m}^{m+k} N_{m}^{m+k}\right)_{r_{m}}, \\
& C=-\frac{m}{s \nu} \sum_{k \neq 0} \frac{1}{k}\left(N_{m}^{m+k} \bar{Z}_{m+k}+P_{m}^{m+k} \bar{\psi}_{m+k}\right)_{r_{m}}, \\
& D=C-\sum_{k \neq 0} \frac{1}{k}\left(L_{m}^{m+k} \bar{Z}_{m+k}+M_{m}^{m+k} \bar{\psi}_{m+k}\right)_{r_{m}}, \\
& a_{k}=\frac{1}{m}\left(\frac{N_{m}^{m+k}}{s \nu}+\frac{L_{m}^{m+k}}{m}\right)_{r_{m}}, \\
& \tilde{a}_{k}=\frac{1}{m}\left(\frac{N_{m}^{m+k}}{s}+\frac{L_{m}^{m+k}}{m}\right)_{r_{m}}, \\
& b_{k}=-\frac{1}{m}\left(\frac{M_{m}^{m+k}}{m}+\frac{P_{m}^{m+k}}{s \nu}\right)_{r_{m}},
\end{aligned}
$$




$$
\tilde{b}_{k}=-\frac{1}{m}\left(\frac{M_{m}^{m+k}}{m}+\frac{P_{m}^{m+k}}{s}\right)_{r_{m}}
$$

The parameter

$$
\kappa=\left(\frac{L_{m}^{m}}{m^{2}} \frac{r}{s} \frac{d P_{m}^{m}}{d r}\right)_{r_{m}} \simeq\left(-q r \frac{d}{d r}\left[\frac{1}{r} \frac{d}{d r}\left(\frac{r^{2}}{q}\right)\right]\right)_{r_{m}}+\mathcal{O}\left(\epsilon^{2}\right)
$$

is a measure of the local equilibrium toroidal current gradient. The relationship between $A^{L}$, $B^{L}$ and $A^{R}, B^{R}$ is determined by asymptotically matching Eqs. (A.7) across a thin nonideal layer centered on the rational surface. In the high mode number limit, $m \gg 1$, the expansion (A.7) is valid provided $\delta \epsilon^{2} m \ll 1$, where $\delta$ is the layer width (normalized to $r_{m}$ ).

\section{Asymptotic matching between the inner and outer regions}

The region of the plasma governed by the ideal-MHD equations is usually termed the outer region, whereas the thin layer centered on the rational surface where ideal-MHD breaks down is termed the inner region. ${ }^{8}$ Asymptotic matching between the inner and outer regions is conventionally performed using the normalized displacement

$$
\phi_{m}(x)=-m s B_{0} f \xi_{m}(x)
$$

where

$$
\boldsymbol{\xi} \cdot \nabla r=\sum_{m} \xi_{m} \exp [\mathrm{i}(m \theta-n \phi)]
$$

and $\boldsymbol{\xi}$ is the actual plasma displacement. In the outer region $\phi_{m}=\psi_{m} / x$.

The layer equations in the inner region are most easily solved in Fourier transform space, ${ }^{10}$ so it is convenient to write

$$
\phi_{m}(x)=\int_{-\infty}^{\infty} \hat{\phi}_{m}(k) \exp (\mathrm{i} k x) d k
$$

The layer equations are assumed to be parity conserving (see Appendix B). ${ }^{10}$ This is reasonable because the principle parity breaking effects, which are associated with radial gradients 
in the equilibrium plasma current and pressure, are generally not important in the layer. Let

$$
\widehat{\phi}_{m}(k)=\frac{1}{2} \widehat{\phi}_{m}^{+}(|k|)-\frac{\mathrm{i}}{2} \widehat{\phi}_{m}^{-}(|k|) \operatorname{sgn}(k),
$$

where the even-parity and odd-parity displacements are completely independent. The most general small- $k$ asymptotic behavior of the Fourier transformed displacement is ${ }^{6}$

$$
\begin{aligned}
& \hat{\phi}_{m}^{+}(|k|) \simeq \frac{1}{\pi} B^{-}|k|^{\nu-1}+A^{-}|k|^{-\nu}+\cdots, \\
& \hat{\phi}_{m}^{-}(|k|) \simeq \frac{1}{\pi} B^{+}|k|^{\nu-1}+A^{+}|k|^{-\nu}+\cdots .
\end{aligned}
$$

The two ratios

$$
\Delta^{ \pm}(\omega)=\frac{B^{ \pm}}{A^{ \pm}}
$$

are completely determined by the solution of the even and odd parity Fourier transformed layer equations, and are in general functions of the mode rotation frequency $\omega$ [where all layer quantities are assumed to vary like exp $(-i \omega t)]$ (see Appendix B). In configuration space Eqs. (A.15) yield the following asymptotic behavior of the inner solution as it merges into the outer region:

$$
\phi_{m}(x)=\phi_{m}^{+}(|x|)+\phi_{m}^{-}(|x|) \operatorname{sgn}(x)
$$

where

$$
\begin{aligned}
& \phi_{m}^{+}(|x|) \simeq\left(\frac{\nu \pi}{2}\right) A^{-}|x|^{\nu-1}+\frac{1}{2}\left(\frac{\nu \pi}{2}\right)^{-1} B^{-}|x|^{-\nu}+\cdots, \\
& \phi_{m}^{-}(|x|) \simeq A^{+}|x|^{\nu-1}+\frac{1}{2} B^{+}|x|^{-\nu}+\cdots
\end{aligned}
$$

for $|\nu| \ll 1$, or

$$
\psi_{m}(x)=\psi_{m}^{+}(|x|)+\psi_{m}^{-}(|x|) \operatorname{sgn}(x)
$$

where

$$
\begin{aligned}
& \psi_{m}^{+}(|x|) \simeq A^{+}|x|^{\nu}+\frac{1}{2} B^{+}|x|^{1-\nu}+\cdots \\
& \psi_{m}^{-}(|x|) \simeq\left(\frac{\nu \pi}{2}\right) A^{-}|x|^{\nu}+\frac{1}{2}\left(\frac{\nu \pi}{2}\right)^{-1} B^{-}|x|^{1-\nu}+\cdots
\end{aligned}
$$


Asymptotic matching of Eqs. (A.7) and (A.19)-(A.20) yields the following expression for the solution of the ideal-MHD equations in the vicinity of a rational surface:

$$
\begin{aligned}
\psi_{m} \simeq & {\left[A^{+}+\left(\frac{\nu \pi}{2}\right) A^{-} \operatorname{sgn}(x)\right]|x|^{\nu}[1+\lambda x] } \\
& +\frac{1}{2}\left[B^{+}+\left(\frac{\nu \pi}{2}\right)^{-1} B^{-} \operatorname{sgn}(x)\right]|x|^{1-\nu}+C x+\cdots, \\
L_{0} Z_{m} \simeq & {\left[A^{+}+\left(\frac{\nu \pi}{2}\right) A^{-} \operatorname{sgn}(x)\right]|x|^{\nu}[\nu+\gamma x] } \\
& +\frac{1}{2}\left[B^{+}+\left(\frac{\nu \pi}{2}\right)^{-1} B^{-} \operatorname{sgn}(x)\right](1-\nu)|x|^{1-\nu}+D x+\cdots, \\
\psi_{m+k} \simeq & {\left[A^{+}+\left(\frac{\nu \pi}{2}\right) A^{-} \operatorname{sgn}(x)\right] a_{k}|x|^{\nu} } \\
& +\left[B^{+}+\left(\frac{\nu \pi}{2}\right)^{-1} B^{-} \operatorname{sgn}(x)\right] \tilde{u}_{k}|x|^{1-\nu}+\bar{\psi}_{m+k}+\cdots \\
Z_{m+k} \simeq & {\left[A^{+}+\left(\frac{\nu \pi}{2}\right) A^{-} \operatorname{sgn}(x)\right] b_{k}|x|^{\nu} } \\
& +\left[B^{+}+\left(\frac{\nu \pi}{2}\right)^{-1} B^{-} \operatorname{sgn}(x)\right] \tilde{b}_{k}|x|^{1-\nu}+\bar{Z}_{m+k}+\cdots,
\end{aligned}
$$

where $A^{ \pm}$and $B^{ \pm}$are related via Eqs. (A.16).

According to Eq. (A.8) the Mercier index $\nu$ is $\mathcal{O}\left(\epsilon^{2}\right)$ in the adopted ordering scheme. It can be seen that there are many terms in Eqs. (A.9) and (A.21) which depend on $\nu^{-1}$, suggesting that the zero curvature limit $\nu \rightarrow 0$ is nonuniform. In fact, this limit is perfectly well behaved, as is easily demonstrated by making the substitutions

$$
\begin{aligned}
\bar{\psi}_{m+k} & =-A^{+} a_{k}+\tilde{\psi}_{m+k}, \\
\bar{Z}_{m+k} & =-A^{+} b_{k}+\tilde{Z}_{m+k}, \\
B^{+} & =-\frac{\pi}{2} \frac{\kappa}{s} A^{-}+\hat{B}^{+},
\end{aligned}
$$




$$
\begin{aligned}
B^{-}= & -\frac{\pi}{2} \frac{\kappa}{s} A^{+}+\frac{\pi m}{s} \sum_{k \neq 0} \frac{1}{k}\left(N_{m}^{m+k} \tilde{Z}_{m+k}+P_{m}^{m+k} \tilde{\psi}_{m+k}\right)_{r_{m}} \\
& -\pi \nu\left[\frac{\kappa}{s}+\frac{1}{2}+\frac{1}{2}\left(\frac{r q^{\prime \prime}}{q^{\prime}}\right)_{r_{m}}\right] A^{+}+\frac{\pi \nu}{2} \hat{B}^{-},
\end{aligned}
$$

and expanding in small- $\nu$, after which Eqs. (A.21) reduce to

$$
\begin{gathered}
\psi_{m} \simeq A^{+}[1+\hat{\lambda} x(\ln |x|-1)]+A^{-} \tilde{\lambda}|x|+\frac{1}{2}\left(\hat{B}^{+}|x|+\hat{B}^{-} x\right) \\
+\hat{C} x+\widehat{D} x(\ln |x|-1)+\mathcal{O}\left(x^{2}\right)+\mathcal{O}(\nu) \\
L_{0} Z_{m} \simeq A^{+} \hat{\gamma} x \ln |x|+\frac{1}{2}\left(\hat{B}^{+}|x|+\hat{B}^{-} x\right)+\widehat{D} x \ln |x|+\mathcal{O}\left(x^{2}\right)+\mathcal{O}(\nu) \\
\psi_{m+k} \simeq\left[A^{+} \ln |x|+\frac{\pi}{2} A^{-} \operatorname{sgn}(x)\right] \widehat{a}_{k}+\tilde{\psi}_{m+k}+\mathcal{O}(x)+\mathcal{O}(\nu) \\
Z_{m+k} \simeq\left[A^{+} \ln |x|+\frac{\pi}{2} A^{-} \operatorname{sgn}(x)\right] \hat{b}_{k}+\tilde{Z}_{m+k}+\mathcal{O}(x)+\mathcal{O}(\nu)
\end{gathered}
$$

in the zero curvature limit. In the above,

$$
\begin{aligned}
& \hat{\lambda}=\frac{\kappa}{s}-\frac{1}{m s} \sum_{k \neq 0} \frac{1}{k}\left(L_{m}^{m+k} P_{m}^{m+k}-M_{m}^{m+k} N_{m}^{m+k}\right)_{r_{m}} \\
& \tilde{\lambda}=-\frac{\pi}{2} \frac{1}{m s} \sum_{k \neq 0} \frac{1}{k}\left(L_{m}^{m+k} P_{m}^{m+k}-M_{m}^{m+k} N_{m}^{m+k}\right)_{r_{m}} \\
& \hat{\gamma}=\frac{\kappa}{s} \\
& \widehat{C}=\sum_{k \neq 0} \frac{1}{k}\left(L_{m}^{m+k} \tilde{Z}_{m+k}+M_{m}^{m+k} \tilde{\psi}_{m+k}\right)_{r_{m}}, \\
& \widehat{D}=-\frac{m}{s} \sum_{k \neq 0} \frac{1}{k}\left(N_{m}^{m+k} \tilde{Z}_{m+k}+P_{m}^{m+k} \tilde{\psi}_{m+k}\right)_{r_{m}} \\
& \hat{a}_{k}=\frac{\left(N_{m}^{m+k}\right)_{r_{m}}}{m s} \\
& \hat{b}_{k}=-\frac{\left(P_{m}^{m+k}\right)_{r_{m}}}{m s} .
\end{aligned}
$$




\section{The dispersion relation for coupled tearing and twisting modes}

Suppose that there are $N$ rational surfaces in the plasma radii $r_{1}<r_{2} \cdots<r_{N}$, resonant with poloidal mode numbers $m_{1}, m_{2} \cdots m_{N}$ (for a fixed toroidal mode number $n$ ). The toroidal electromagnetic torque acting in the vicinity of rational surface $j$ is given by

$$
\delta T_{\phi}\left(r_{j}\right)=\left[\oint \oint R^{2} \nabla \phi \cdot \delta \mathbf{J} \wedge \delta \mathbf{B} j d \theta d \phi\right]_{r_{j-}}^{r_{j+}}
$$

where $r_{j \pm}$ is the outer/inner radius of the associated nonideal layer, and $\delta \mathbf{J}$ is the perturbed current. It can be shown that ${ }^{4}$

$$
\begin{aligned}
\delta T_{\phi}\left(r_{j}\right) & =\frac{2 n \pi^{2} R_{0}}{\mu_{0}} \times\left[\sum_{k} \frac{\operatorname{Im}\left\{\left(\psi_{k}\right)^{*} Z_{k}\right\}}{(k-n q)}\right]_{r_{j-}}^{r_{j+}} \\
& =\frac{2 n \pi^{2} R_{0}}{\mu_{0}} \times\left[\frac{\left(1-2 \nu_{j}\right)}{L_{m_{j}}^{m_{j}}}\right]_{r_{j}} \times \operatorname{Im}\left[\left(A^{R}\right)^{*} B^{R}+\left(A^{L}\right)^{*} B^{L}\right]_{j} \\
& =\frac{2 n \pi^{2} R_{0}}{\mu_{0}} \times\left[\frac{\left(1-2 \nu_{j}\right)}{L_{m_{j}}^{m_{j}}}\right]_{r_{j}} \times \operatorname{Im}\left[\left(A^{+}\right)^{*} B^{+}+\left(A^{-}\right)^{*} B^{-}\right]_{j},
\end{aligned}
$$

where $\nu_{j}$ is the Mercier index for surface $j$ [see Eq. (A.8)], and where $\left(A^{L, R}\right)_{j},\left(B^{L, R}\right)_{j},\left(A^{ \pm}\right)_{j}$, and $\left(B^{ \pm}\right)_{j}$ are coefficients in the expansions (A.7) and (A.21) evaluated in the vicinity of this surface.

It is useful to define the quantities ${ }^{4}$

$$
\begin{gathered}
\Psi_{j}^{ \pm}=\left[\frac{\left(1-2 \nu_{j}\right)}{L_{m_{j}}^{m_{j}}}\right]_{r_{j}}^{1 / 2}\left(A^{ \pm}\right)_{j}, \\
\Delta \Psi_{j}^{ \pm}=\left[\frac{\left(1-2 \nu_{j}\right)}{L_{m_{j}}^{m_{j}}}\right]_{r_{j}}^{1 / 2}\left(B^{ \pm}\right)_{j},
\end{gathered}
$$

where $\Psi_{j}^{+}$is termed the 'tearing amplitude' at surface $j$ and $\Psi_{j}^{-}$is the associated 'twisting amplitude.' The tearing amplitude is sometimes referred to as the 'reconnected flux.' It follows from (A.16) that

$$
\Delta_{j}^{ \pm}(\omega)=\frac{\Delta \Psi_{j}^{ \pm}}{\Psi_{j}^{ \pm}},
$$


where $\Delta_{j}^{+}(\omega)$ is the tearing parity layer dispersion relation at surface $j$, and $\Delta_{j}^{-}(\omega)$ is the associated twisting parity dispersion relation. Equation (A.26) simplifies to

$$
\begin{aligned}
\delta T_{\phi}\left(r_{j}\right) & =\frac{2 n \pi^{2} R_{0}}{\mu_{0}} \times \operatorname{Im}\left[\left(\Psi_{j}^{+}\right)^{*} \Delta \Psi_{j}^{+}+\left(\Psi_{j}^{-}\right)^{*} \Delta \Psi_{j}\right] \\
& =\frac{2 n \pi^{2} R_{0}}{\mu_{0}} \times\left[\operatorname{Im}\left(\Delta_{j}^{+}\right)\left|\Psi_{j}^{+}\right|^{2}+\operatorname{Im}\left(\Delta_{j}^{-}\right)\left|\Psi_{j}^{-}\right|^{2}\right] .
\end{aligned}
$$

The system has $2 N$ degrees of freedom (i.e. two degrees for each rational surface in the plasma), so a general mode can be built up from a linear superposition of $2 N$ independent basis modes. It is convenient to define $N$ basis tearing modes, denoted $\psi_{j}^{+}$(for $j=1$ to $N$ ). These are solutions of Eqs. (A.6) which satisfy the physical boundary conditions at $r=0$ and $r=a$ and are subject to the additional constraints:

$$
\begin{gathered}
\Psi_{k}^{+}=\delta_{k j}, \\
\Psi_{k}^{-}=0, \\
\Delta \Psi_{k}^{+}=E_{k j}^{+}, \\
\Delta \Psi_{k}^{-}=H_{k j}^{-} .
\end{gathered}
$$

Thus, the $j$ th basis tearing mode has unit tearing amplitude and zero twisting amplitude at surface $j$, with zero tearing or twisting amplitude at any other surface. It is also convenient to define $N$ basis twisting modes, denoted $\boldsymbol{\psi}_{j}^{-}$. These are solutions of Eqs. (A.6) which satisfy the physical boundary conditions and are subject to the constraints:

$$
\begin{gathered}
\Psi_{k}^{+}=0, \\
\Psi_{k}^{-}=\delta_{k j}, \\
\Delta \Psi_{k}^{+}=H_{k j}^{+},
\end{gathered}
$$




$$
\Delta \Psi_{k}^{-}=E_{k j}^{-} .
$$

Thus, the $j$ th basis twisting mode has unit twisting amplitude and zero tearing amplitude at surface $j$, with zero tearing or twisting amplitude at any other surface. Note that the quantities $E_{k j}^{ \pm}$and $H_{k j}^{ \pm}$must be real because the ideal-MHD equations (A.6) contain no complex coefficients.

A general mode is written

$$
\psi=\sum_{k=1}^{N}\left(\Psi_{k}^{+} \psi_{k}^{+}+\Psi_{k}^{-} \psi_{k}^{-}\right)
$$

yielding

$$
\begin{aligned}
& \Delta \Psi_{j}^{+}=\sum_{k=1}^{N}\left(\Psi_{k}^{+} E_{j k}^{+}+\Psi_{k}^{-} H_{j k}^{+}\right), \\
& \Delta \Psi_{j}^{-}=\sum_{k=1}^{N}\left(\Psi_{k}^{+} H_{j k}^{-}+\Psi_{k}^{-} E_{j k}^{-}\right) .
\end{aligned}
$$

Conservation of toroidal angular momentum requires that there is zero net.toroidal electromagnetic torque acting on an isolated plasma. ${ }^{4}$ So, making use of (A.29),

$$
\begin{aligned}
T_{\phi}= & \sum_{j=1}^{N} \delta T_{\phi}\left(r_{j}\right)=\frac{2 n \pi^{2} R_{0}}{\mu_{0}} \times \sum_{j, k=1}^{N} \operatorname{Im}\left[E_{j k}^{+}\left(\Psi_{j}^{+}\right)^{*} \Psi_{k}^{+}+E_{j k}^{-}\left(\Psi_{j}^{-}\right)^{*} \Psi_{k}^{-}\right. \\
& \left.+H_{j k}^{+}\left(\Psi_{j}^{+}\right)^{*} \Psi_{k}^{-}+H_{j k}^{-}\left(\Psi_{j}^{-}\right)^{*} \Psi_{k}^{+}\right]=0,
\end{aligned}
$$

which implies that

$$
\begin{aligned}
& E_{j k}^{+}=E_{k j}^{+}, \\
& E_{j k}^{-}=E_{k j}^{-}, \\
& H_{j k}^{+}=H_{k j}^{-}=H_{j k} .
\end{aligned}
$$


The general resistive mode dispersion relation can be expressed as two coupled matrix equations:

$$
\begin{gathered}
\left\{\Delta^{+}(\omega)-\mathbf{E}^{+}\right\} \Psi^{+}-\mathbf{H} \Psi^{-}=0 \\
\left\{\Delta^{-}(\omega)-\mathbf{E}^{-}\right\} \Psi^{-}-\mathbf{H}^{\dagger} \Psi^{+}=0
\end{gathered}
$$

where use has been made of (A.28), (A.33), and (A.35). In the above, $\mathbf{E}^{ \pm}$is the $N \times N$ real symmetric matrix of the $E_{i j}^{ \pm}$values, $\mathbf{H}$ is the $N \times N$ real matrix of the $H_{i j}$ values and $\mathbf{H}^{\dagger}$ is its transpose, $\Delta^{ \pm}(\omega)$ is the $N \times N$ complex diagonal matrix of the $\Delta_{j}^{ \pm}(\omega)$ values, and $\Psi^{ \pm}$ is the $1 \times N$ complex vector of the $\Psi_{j}^{ \pm}$values.

\section{Basis tearing modes}

The construction of the basis tearing mode solutions and evaluation of the elements of the $\mathrm{E}^{+}$Matrix, using the T7 code, is described in FHMR. Consider the $j$ th basis tearing mode solution, satisfying (A.30). According to (A.22d)

$$
\begin{aligned}
\Delta \Psi_{j}^{-}= & -\frac{\pi}{2}\left[\frac{\kappa_{j}}{s_{j}}\left(1+2 \nu_{j}\right)+\nu_{j}+\nu_{j}\left(\frac{r q^{\prime \prime}}{q^{\prime}}\right)_{r_{j}}\right] \\
& +\frac{\pi}{s_{j}} \sum_{k \neq 0} \frac{1}{k}\left(N_{m_{j}}^{m_{j}+k} \tilde{Z}_{m_{j}+k}+P_{m_{j}}^{m_{j}+k} \tilde{\psi}_{m_{j}+k}\right)_{r_{j}}+\frac{\pi}{2} \frac{\nu_{j}}{m_{j}}\left(\hat{B}^{-}\right)_{j}+\mathcal{O}\left(\epsilon^{3}\right),
\end{aligned}
$$

where use has been made of Eqs. (A.27) and $L_{m_{j}}^{m_{j}}=m_{j}^{2}+\mathcal{O}\left(\epsilon^{2}\right) .{ }^{4}$ Here, $\kappa_{j}$ is the current gradient parameter evaluated at the $j$ th rational surface [see Eq. $\left(\right.$ A.10)], and $s_{j}=\left(r q^{\prime} / q\right)_{r_{j}}$ is the local magnetic shear. It can be shown that ${ }^{4}$

$$
\begin{aligned}
\left(N_{m_{j}}^{m_{j} \pm 1}\right)_{r_{j}} & =\frac{1}{2}\left(m_{j} \pm 1\right) \alpha_{j}, \\
\left(P_{m_{j}}^{m_{j} \pm 1}\right)_{r_{j}} & =\frac{1}{2}\left(1+s_{j}\right) \alpha_{j},
\end{aligned}
$$

where

$$
\alpha_{j}=-\left(\frac{2 R_{0} \mu_{0} p^{\prime} q^{2}}{B_{0}^{2}}\right)_{r_{j}}
$$


parameterizes the local pressure gradient at rational surface $j$. It can also be shown that $\left(N_{m_{j}}^{m_{j} \pm k}\right)_{r_{j}}=\left(P_{m_{j}}^{m_{j} \pm k}\right)_{r_{j}}=0$ for $k>1 .^{4}$ It follows that

$$
\begin{aligned}
H_{j j}=\Delta \Psi_{j}^{-}= & -\frac{\pi}{2}\left[\frac{\kappa_{j}}{s_{j}}\left(1+2 \nu_{j}\right)+\nu_{j}+\nu_{j}\left(\frac{r q^{\prime \prime}}{q^{\prime}}\right)_{r_{j}}\right] \\
& +\frac{\pi}{2} \frac{\alpha_{j}}{s_{j}} \sum_{ \pm}( \pm)\left[\left(m_{j} \pm 1\right) Z_{m_{j} \pm 1}\left(r_{j}\right)+\left(1+s_{j}\right) \psi_{m_{j} \pm 1}\left(r_{j}\right)\right] \\
& -\frac{\pi}{2} \frac{\nu_{j}}{s_{j}}\left[\frac{Z_{m_{j}}\left(r_{j}+\delta\right)-Z_{m_{j}}\left(r_{j}-\delta\right)+2 \kappa_{j} \delta \ln \delta}{\delta}\right]+\mathcal{O}\left(\epsilon^{3}\right)
\end{aligned}
$$

in the limit $\delta \rightarrow 0$, where use has been made of Eqs. (A.23), (A.24), and (A.30). Thus, the $j$ th diagonal element of the $\mathrm{H}$ Matrix can be evaluated from the $j$ th basis tearing mode solution by reading off the values of the toroidally coupled sidebands at the rational surface [i.e. $\psi_{m_{j} \pm 1}\left(r_{j}\right)$ and $Z_{m_{j} \pm 1}\left(r_{j}\right)$ ] and the value of $Z_{m_{j}}$ at two points which straddle this surface [i.e. $Z_{m_{j}}\left(r_{j} \pm \delta\right)$ ].

Consider the behavior of the $j$ th basis tearing mode solution in the vicinity of rational surface $k$ (where $k \neq j$ ). According to (A.22d), (A.27), and (A.30)

$$
\Delta \Psi_{\bar{k}}^{-}=\frac{\pi}{s_{k}} \frac{1}{m_{j}-m_{k}}\left(N_{m_{k}}^{m_{j}} \tilde{Z}_{m_{j}}+P_{m_{k}}^{m_{j}} \tilde{\psi}_{m_{j}}\right)_{r_{k}}+\mathcal{O}\left(\epsilon^{2}\right)
$$

assuming $m_{j} \neq m_{k}$. Thus, making use of (A.23), (A.30), (A.35), and (A.38)

$$
H_{j k}=\Delta \Psi_{k}^{-}=\mp \frac{\pi}{2} \frac{\alpha_{k}}{s_{k}}\left[m_{j} Z_{m_{j}}\left(r_{k}\right)+\left(1+s_{k}\right) \psi_{m_{j}}\left(r_{k}\right)\right]+\mathcal{O}\left(\epsilon^{2}\right)
$$

for $m_{k}=m_{j} \pm 1$, and $H_{j k}=0$ for $m_{k} \neq m_{j} \pm 1$.

In Eq. (A.42) the functions $Z_{m_{j}}$ and $\psi_{m_{j}}$ need only be evaluated to leading (i.e. cylindrical) order in $\epsilon$. Now, in the cylindrical limit

$$
\begin{aligned}
& L_{m}^{m}=m^{2}, \\
& P_{m}^{m}=(m-n q)^{2}-\frac{(m-n q)}{m} \kappa_{0},
\end{aligned}
$$


where

$$
\kappa_{0}(r)=-q r \frac{d}{d r}\left[\frac{1}{r} \frac{d}{d r}\left(\frac{r^{2}}{q}\right)\right] .
$$

Thus, the ideal-MHD equations (A.6) reduce to

$$
Z_{m}(r)=\frac{(m-n q)}{m^{2}} r \frac{d \psi_{m}}{d r}
$$

and

$$
r \frac{d}{d r}\left(r \frac{d \psi_{m}}{d r}\right)-m^{2} \psi_{m}+\frac{m \kappa_{0}}{(m-n q)} \psi_{m}=0 .
$$

The above equation is usually referred to as the 'cylindrical tearing mode equation.'

Let $\psi_{m_{j}}^{C}(r)$ be a solution of the cylindrical tearing mode equation (with $m=m_{j}$ ) which satisfies the physical boundary conditions at $r=0$ and $r=a$, and is subject to the constraint

$$
\psi_{m_{j}}^{C}\left(r_{j}\right)=1
$$

It follows from (A.27), (A.42), (A.43), (A.45), and (A.47) that

$$
H_{j k}=\frac{\pi}{2} \frac{\alpha_{k}}{s_{k}}\left[r_{k} \frac{d \psi_{m_{j}}^{C}\left(r_{k}\right)}{d r} \mp\left(1+s_{k}\right) m_{j} \psi_{m_{j}}^{C}\left(r_{k}\right)\right]+\mathcal{O}\left(\epsilon^{2}\right)
$$

for $m_{k}=m_{j} \pm 1$, and $H_{j k}=0$ for $m_{k} \neq m_{j} \pm 1$. Thus, the off-diagonal elements of the $\mathrm{H}$ Matrix can be evaluated using standard cylindrical basis functions.

\section{Basis twisting modes}

Consider the $j$ th basis twisting mode solution, satisfying (A.31). According to (A.23), (A.24), (A.27), and (A.38) the resonant harmonic is $\mathcal{O}\left(\epsilon^{2}\right)$, whereas the two toroidally coupled sidebands are $\mathcal{O}(\epsilon)$ and satisfy ${ }^{6}$

$$
\begin{aligned}
& {\left[\psi_{m_{j} \pm 1}\right]_{r_{j-}}^{r_{j+}}=\frac{\pi}{2} \frac{\alpha_{j}}{s_{j}}\left(m_{j} \pm 1\right),} \\
& {\left[Z_{m_{j} \pm 1}\right]_{r_{j-}}^{r_{j+}}=-\frac{\pi}{2} \frac{\alpha_{j}}{s_{j}}\left(1+s_{j}\right) .}
\end{aligned}
$$


In fact, the toroidally coupled sidebands can be constructed from cylindrical basis functions which obey

$$
\begin{gathered}
{\left[\psi_{m_{j} \pm 1}\right]_{r_{j-}}^{r_{j+}}=\frac{\pi}{2} \frac{\alpha_{j}}{s_{j}}\left(m_{j} \pm 1\right),} \\
{\left[r \frac{d \psi_{m_{j} \pm 1}}{d r}\right]_{r_{j-}}^{r_{j+}}=\mp \frac{\pi}{2} \frac{\alpha_{j}}{s_{j}}\left(m_{j} \pm 1\right)^{2}\left(1+s_{j}\right),}
\end{gathered}
$$

where use has been made of (A.45).

Let $\psi_{m_{j} \pm 1}^{L}(r)$ be a solution of the cylindrical tearing mode equation (A.46) (for poloidal mode number $m_{j} \pm 1$ ) in the interval $0 \leq r<r_{j}$ which satisfies the physical boundary conditions at $r=0$, and is subject to the constraints

$$
\begin{aligned}
& \psi_{m_{j} \pm 1}^{L}\left(r_{L_{j}}\right)=0, \\
& \psi_{m_{j} \pm 1}^{L}\left(r_{j-}\right)=1 .
\end{aligned}
$$

Here, the $r_{L_{j}}$ are the radii of any rational surfaces resonant with poloidal mode number $m_{j} \pm 1$ which lie in the interval $0 \leq r<r_{j}$. Likewise, let $\psi_{m_{j} \pm 1}^{R}(r)$ be a solution of the cylindrical tearing mode equation in the interval $r>r_{j}$ which satisfies the physical boundary conditions at $r=a$, and is subject to

$$
\begin{aligned}
& \psi_{m_{j} \pm 1}^{R}\left(r_{R_{j}}\right)=0 \\
& \psi_{m_{j} \pm 1}^{R}\left(r_{j+}\right)=1 .
\end{aligned}
$$

Here, the $r_{R_{j}}$ are the radii of any rational surfaces resonant with poloidal mode number $m_{j} \pm 1$ which lie in the interval $r>r_{j}$. It is also useful to define the quantities

$$
\begin{aligned}
& \Delta_{m_{j} \pm 1}^{L}=\left[r \frac{d \psi_{m_{j} \pm 1}^{L}}{d r}\right]_{r_{j-}}, \\
& \Delta_{m_{j} \pm 1}^{R}=\left[r \frac{d \psi_{m_{j} \pm 1}^{R}}{d r}\right]_{r_{j+}} .
\end{aligned}
$$


The solution of the cylindrical tearing mode equation which satisfies (A.50) takes the form

$$
\begin{aligned}
\psi_{m_{j} \pm 1}(r)=- & \frac{\pi}{2} \frac{\alpha_{j}}{s_{j}}\left(m_{j} \pm 1\right)\left[\frac{\Delta_{m_{j} \pm 1}^{R} \pm\left(m_{j} \pm 1\right)\left(1+s_{j}\right)}{\Delta_{m_{j} \pm 1}^{R}-\Delta_{m_{j} \pm 1}^{L}} \psi_{m_{j} \pm 1}^{L}(r)\right. \\
& \left.+\frac{\Delta_{m_{j} \pm 1}^{L} \pm\left(m_{j} \pm 1\right)\left(1+s_{j}\right)}{\Delta_{m_{j} \pm 1}^{R}-\Delta_{m_{j} \pm 1}^{L}} \psi_{m_{j} \pm 1}^{R}(r)\right] .
\end{aligned}
$$

According to (A.22d), (A.27), (A.31), (A.38), and (A.45)

$$
\begin{aligned}
E_{j j}^{-}=\Delta \Psi_{j}^{-} & =\frac{\pi}{s_{j}} \sum_{k \neq 0} \frac{1}{k}\left(N_{m_{j}}^{m_{j}+k} \tilde{Z}_{m_{j}+k}+P_{m_{j}}^{m_{j}+k} \tilde{\psi}_{m_{j}+k}\right)_{r_{j}}+\mathcal{O}\left(\epsilon^{3}\right) \\
& =\frac{\pi}{2} \frac{\alpha_{j}}{s_{j}} \sum_{ \pm}\left[\frac{1}{m_{j} \pm 1} r_{j} \frac{d \psi_{m_{j} \pm 1}\left(r_{j}\right)}{d r} \pm\left(1+s_{j}\right) \psi_{m_{j} \pm 1}\left(r_{j}\right)\right]+\mathcal{O}\left(\epsilon^{3}\right) .
\end{aligned}
$$

Thus, ${ }^{6}$

$$
\begin{aligned}
E_{j j}^{-}= & -\frac{\pi^{2}}{4} \frac{\alpha_{j}^{2}}{s_{j}^{2}} \times \\
& \sum_{ \pm} \frac{\Delta_{m_{j} \pm 1}^{R} \Delta_{m_{j} \pm 1}^{L} \pm\left(m_{j} \pm 1\right)\left(1+s_{j}\right)\left(\Delta_{m_{j} \pm 1}^{R}+\Delta_{m_{j} \pm 1}^{L}\right)+\left(m_{j} \pm 1\right)^{2}\left(1+s_{j}\right)^{2}}{\Delta_{m_{j} \pm 1}^{R}-\Delta_{m_{j} \pm 1}^{L}}
\end{aligned}
$$

where use has been made of (A.54). It can also be shown that $E_{k j}^{-}=\mathcal{O}\left(\epsilon^{3}\right)$ for $m_{k} \neq m_{j}$, so for a monotonic $q$-profile the $\mathrm{E}^{-}$Matrix is diagonal to $\mathcal{O}\left(\epsilon^{2}\right)$. Equation (A.22c) yields

$$
H_{j j}=\Delta \Psi_{j}^{+}=-\frac{\pi}{2} \frac{\kappa_{j}}{s_{j}}+\mathcal{O}\left(\epsilon^{2}\right)
$$

which is consistent with (A.40).

It follows from (A.23), (A.27), (A.30), (A.35), and (A.54) that

$$
H_{k j}=\Delta \Psi_{k}^{+}=-\frac{\pi}{2} \frac{\alpha_{j}}{s_{j}} \frac{\Delta_{m_{k}}^{R}-m_{k}\left(1+s_{j}\right)}{\Delta_{m_{k}}^{R}-\Delta_{m_{k}}^{L}} r_{k} \frac{d \psi_{m_{k}}^{L}\left(r_{k}\right)}{d r}+\mathcal{O}\left(\epsilon^{2}\right)
$$

provided $m_{k}=m_{j}-1$ and $r_{k}<r_{j}$. It is easily demonstrated that

$$
\left[r \frac{d \psi_{m}^{a}}{d r} \psi_{m}^{b}-r \frac{d \psi_{m}^{b}}{d r} \psi_{m}^{a}\right]_{r_{1}}^{r_{2}}=0
$$


where $\psi_{m}^{a}$ and $\psi_{m}^{b}$ are two solutions of the cylindrical tearing mode equation (A.46) (for poloidal mode number $m$ ) which are continuous in the interval $r_{1}$ to $r_{2}$. Equation (A.59) can be used to show that

$$
\begin{aligned}
r_{k} \frac{d \psi_{m_{k}}^{L}\left(r_{k}\right)}{d r} & =-\left(\Delta_{m_{k}}^{L}-\Delta_{m_{k}}^{R}\right) \psi_{m_{k}}^{C}\left(r_{j}\right) \\
\Delta_{m_{k}}^{R} \psi_{m_{k}}^{C}\left(r_{j}\right) & =r_{j} \frac{d \psi_{m_{k}}^{C}\left(r_{j}\right)}{d r} .
\end{aligned}
$$

Thus, (A.58) reduces to

$$
H_{k j}=\frac{\pi}{2} \frac{\alpha_{j}}{s_{j}}\left[r_{j} \frac{d \psi_{m_{k}}^{C}\left(r_{j}\right)}{d r}-\left(1+s_{j}\right) m_{k} \psi_{m_{k}}^{C}\left(r_{j}\right)\right]+\mathcal{O}\left(\epsilon^{2}\right)
$$

which is consistent with (A.48).

\section{Conclusions}

The general dispersion relation for tearing and twisting modes in a plasma containing $N$ rational surfaces takes the form:

$$
\begin{gathered}
\left\{\boldsymbol{\Delta}^{+}(\omega)-\mathbf{E}^{+}\right\} \boldsymbol{\Psi}^{+}-\mathbf{H} \boldsymbol{\Psi}^{-}=\mathbf{0} \\
\left\{\boldsymbol{\Delta}^{-}(\omega)-\mathbf{E}^{-}\right\} \boldsymbol{\Psi}^{-}-\mathbf{H}^{\dagger} \boldsymbol{\Psi}^{+}=\mathbf{0}
\end{gathered}
$$

where $\mathbf{E}^{ \pm}$is an $N \times N$ real symmetric matrix, $\mathbf{H}$ is an $N \times N$ real matrix and $\mathbf{H}^{\dagger}$ is its transpose, $\Delta^{ \pm}(\omega)$ is the $N \times N$ complex diagonal matrix of the $\Delta_{j}^{ \pm}(\omega)$ values, and $\boldsymbol{\Psi}^{ \pm}$is the $1 \times N$ complex vector of the $\Psi_{j}^{ \pm}$values. Here, $\Delta_{j}^{+}(\omega)$ is the tearing parity dispersion relation for the layer at rational surface $j$, and $\Delta_{j}(\omega)$ is the corresponding twisting parity dispersion relation (see Appendix B); $\Psi_{j}^{+}$is the 'tearing amplitude' (i.e. the reconnected flux) at surface $j$, and $\Psi_{j}^{-}$is the associated 'twisting amplitude.'

The $\mathrm{E}^{+}$Matrix, which determines the intrinsic stability and mutual interaction of tearing modes, can only be evaluated by solving the full coupled mode equations (A.6). For a 
low- $\beta$, comparatively weakly shaped plasma this is possible using the T7 code, as described in FHMR. The $\mathrm{E}^{-}$Matrix, which determines the intrinsic stability of twisting modes, and the $\mathrm{H}$ Matrix, which determines the coupling of tearing and twisting modes, are more easily evaluated using a combination of standard cylindrical basis functions and data readily obtained from the $\mathrm{T} 7$ code. It has been demonstrated how all matrix elements appearing in the dispersion relation (A.62) can be evaluated to the accuracy required by the T7 ordering scheme; i.e. diagonal elements to $\mathcal{O}\left(\epsilon^{2}\right)$, and off-diagonal elements to $\mathcal{O}(\epsilon)$ (for plasmas with monotonic $q$-profiles).

The $\mathrm{E}^{-}$Matrix is found to be diagonal (assuming a monotonic $q$-profile), indicating that twisting modes do not couple to one another directly. The diagonal elements are $\mathcal{O}\left(\epsilon^{2}\right)$, and are driven exclusively by the equilibrium pressure gradient. The $\mathrm{H}$ Matrix is found to be tridiagonal, indicating that twisting modes couple to tearing modes with the same poloidal mode number (via the equilibrium current gradient), and also to tearing modes with mode numbers which differ by unity (via the equilibrium pressure gradient).

\section{B Physics of the Inner Region}

\section{Incompressible single-fluid layer theory}

\section{Introduction}

The Ohm's law and vorticity equation in the inner region take the form ${ }^{36}$

$$
\begin{aligned}
\frac{d^{2} \psi}{d x^{2}} & =-\mathrm{i} \omega^{\prime} \tau_{R}(\psi-x \phi), \\
x \frac{d^{2} \psi}{d x^{2}} & =\left(\omega^{\prime} \tau_{H}\right)^{2} \frac{d^{2} \phi}{d x^{2}}-\mathrm{i} \frac{\omega^{\prime} \tau_{H}^{2}}{\tau_{V}} \frac{d^{4} \phi}{d x^{4}},
\end{aligned}
$$

respectively, for a single-fluid, zero- $\beta$, incompressible plasma in cylindrical geometry. Here, the radial perturbations in the magnetic field and plasma displacement are written $\delta B_{r}=$ $\mathrm{i} \psi / r$ and $\xi_{r}=-\phi / m s\left(r_{s}\right) B_{\theta}\left(r_{s}\right)$, respectively. All perturbed quantities are assumed to vary 
like $f(x) \exp \left[\mathrm{i}\left(m \theta-n z / R_{0}-\omega t\right)\right]$, where $(r, \theta, z)$ are standard right-handed cylindrical polar coordinates, $R_{0}$ is the simulated major radius, and $x=\left(r-r_{s}\right) / r_{s}$ is the normalized radial distance from the rațional surface $q\left(r_{s}\right)=m / n$. The safety factor is written $q=r B_{0} / R_{0} B_{\theta}$, where $\left[0, B_{\theta}(r), B_{0}\right]$ is the equilibrium magnetic field, and the magnetic shear is defined $s=r(d q / d r) / q$. The hydromagnetic, resistive, and viscous time-scales at the rational surface are written $\tau_{H}=\left(R_{0} / B_{0}\right) \sqrt{\mu_{0} \rho\left(r_{s}\right)} / n s\left(r_{s}\right), \tau_{R}=\mu_{0} r_{s}^{2} / \eta_{\|}\left(r_{s}\right)$, and $\tau_{V}=r_{s}^{2} \rho\left(r_{s}\right) / \mu_{\perp}\left(r_{s}\right)$, respectively, where $\rho$ is the plasma mass density, $\eta_{\|}$the parallel resistivity, and $\mu_{\perp}$ the perpendicular viscosity. Finally, the Doppler shifted layer frequency is defined $\omega^{\prime}=\omega-\omega_{0}$, where $\omega$ is the externally imposed rotation frequency of perturbed quantities and $\omega_{0}$ is the 'natural' frequency determined by local equilibrium plasma flows.

Note that the layer equations (B.1) are parity conserving; i.e. they are invariant under the transformation $x \rightarrow-x, \phi \rightarrow \pm \phi, \psi \rightarrow \mp \psi$. This implies that the twisting parity mode $[\phi(-x)=\phi(x), \psi(-x)=-\psi(x)]$ completely decouples from the tearing parity mode $[\phi(-x)=-\phi(x), \psi(-x)=\psi(x)]$ inside the layer.

Fourier transforming Eqs. (B.1) yields ${ }^{37}$

$$
\frac{d}{d k}\left(\frac{k^{2}}{k^{2}-\mathrm{i}} \frac{d \hat{\phi}}{d k}\right)=-\mathrm{i}\left(V^{3} S\right) k^{2} \widehat{\phi}+\left(V^{3} S^{2} \nu\right) k^{4} \hat{\phi}
$$

where

$$
\phi(x)=\int_{-\infty}^{\infty} \hat{\phi}(k) \exp (\mathrm{i} k x \sqrt{V S}) d k
$$

and

$$
V=\omega^{\prime} \tau_{H}, \quad S=\tau_{R} / \tau_{H}, \quad \nu=\tau_{H} / \tau_{V} .
$$

In the above it is assumed that $\omega^{\prime}>0$. The dispersion relation for $\omega^{\prime}<0$ is easily obtained by taking the complex conjugate of the $\omega^{\prime}>0$ dispersion relation and making the substitution $\omega^{\prime} \rightarrow-\omega^{\prime}$.

Physically acceptable solutions of Eq. (B.2) must be well behaved in the limit $|k| \rightarrow \infty$. 
The most general small- $k$ asymptotic behavior is

$$
\hat{\phi}^{+}(k) \sim \frac{\Delta^{-}}{\pi \sqrt{V S}} \frac{1}{k}+1+\cdots
$$

for twisting parity modes, and

$$
\hat{\phi}^{-}(k) \sim\left(\frac{\Delta^{+}}{\pi \sqrt{V S}} \frac{1}{k}+1\right) \operatorname{sgn}(k)+\cdots
$$

for tearing parity modes. Here, $\Delta^{+}$is the stability index for tearing parity modes, and $\Delta^{-}$is the index for twisting parity modes (the labeling is conventional). In an incompressible layer both parities are indistinguishable in Fourier transform space [i.e. $\hat{\phi}^{-}(k>0)=\hat{\phi}^{+}(k>0)$ ] so that

$$
\Delta^{+}=\Delta^{-} .
$$

Note that in a compressible layer the two parities can be distinguished in Fourier transform space because of different boundary conditions applied to the perturbed pressure. Thus, (B.7) is only likely to hold when plasma compressibility is negligible.

\section{High viscosity solutions}

Tokamak plasmas are observed to possess anomalously large perpendicular viscosity with an effective momentum diffusivity similar in magnitude to the anomalous energy diffusivity. ${ }^{27,38}$ It follows that $S \nu \gg 1$ (i.e. $\tau_{R} \gg \tau_{V}$ ) is the most physically relevant ordering of layer parameters. ${ }^{19}$

Consider the limit $V^{3} S^{2} \nu \gg 1$, in which Eq. (B.2) reduces to the standard Whittaker form ${ }^{39}$

$$
\frac{d^{2} \chi}{d u^{2}}-\frac{1}{4} u^{2} \chi-a \chi=0
$$

for $|u| \ll\left(V^{3} S^{2} \nu\right)^{1 / 4}$, where $\chi=k \hat{\phi}, a=-\exp (\mathrm{i} \pi / 4)(1 / 2)\left(V^{3} / \nu\right)^{1 / 2}$ and $u=\exp (-\mathrm{i} \pi / 8) \sqrt{2}$ $\left(V^{3} S^{2} \nu\right)^{1 / 4} k$. For small $u$, the solution which is regular as $u \rightarrow \infty$ is written

$$
\chi \sim 1-\exp (-\mathrm{i} \pi / 8) \frac{2 \Gamma\left(\frac{3}{4}+\frac{a}{2}\right)}{\Gamma\left(\frac{1}{4}+\frac{a}{2}\right)}\left(V^{3} S^{2} \nu\right)^{1 / 4} k+\cdots,
$$


from which the dispersion relation

$$
\Delta^{ \pm}=-\exp (\mathrm{i} \pi / 8) \frac{\pi}{2} \frac{\Gamma\left(\frac{1}{4}+\frac{a}{2}\right)}{\Gamma\left(\frac{3}{4}+\frac{a}{2}\right)}(V \nu)^{-1 / 4}
$$

is obtained using Eqs. (B.5)-(B.7). In the limit $|a| \gg 1$ (i.e. $V \gg \nu^{1 / 3}$ ) Eq. (B.10) yields the 'ideal-inertial' result,

$$
\Delta^{ \pm}=-\mathrm{i} \frac{\pi}{V}
$$

in which inertia dominates both resistivity and viscosity in the layer. In the opposite limit $|a| \ll 1$ (i.e. $V \ll \nu^{1 / 3}$ ) Eq. (B.10) yields the 'ideal-viscous' result,

$$
\Delta^{ \pm}=-\exp (\mathrm{i} \pi / 8) \frac{\pi}{2} \frac{\Gamma\left(\frac{1}{4}\right)}{\Gamma\left(\frac{3}{4}\right)}(V \nu)^{-1 / 4}
$$

in which viscosity dominates inertia and resistivity in the layer.

Consider the limit $V^{3} S^{2} \nu \ll 1$, in which Eq. (B.2) reduces to

$$
\frac{d}{d k}\left(\frac{k^{2}}{k^{2}-\mathrm{i}} \frac{d \hat{\phi}}{d k}\right) \simeq 0
$$

for $k \lesssim \mathcal{O}(1)$, with solution

$$
\hat{\phi} \simeq\left(\frac{\mathrm{i}}{\mathrm{k}}+k\right)+\alpha+\cdots
$$

where $\alpha$ is an arbitrary constant. For $k \geq \mathcal{O}(1)$, Eq. (B.2) takes the form

$$
\frac{d^{2} \widehat{\phi}}{d k^{2}} \simeq\left(V^{3} S^{2} \nu\right) k^{4} \widehat{\phi}
$$

The solution which is regular as $k \rightarrow \infty$ has the expansion ${ }^{40}$

$$
\widehat{\phi} \sim 1-\frac{6^{2 / 3} \Gamma\left(\frac{5}{6}\right)}{\Gamma\left(\frac{1}{6}\right)}\left(V^{3} S^{2} \nu\right)^{1 / 6} k+\cdots
$$

for $\left(V^{3} S^{2} \nu\right)^{1 / 6} k \ll 1$. Equations (B.5)-(B.7), (B.14), and (B.16) yield the 'visco-resistive' dispersion relation,

$$
\Delta^{ \pm}=-\mathrm{i} 6^{2 / 3} \pi \frac{\Gamma\left(\frac{5}{6}\right)}{\Gamma\left(\frac{1}{6}\right)} V S^{5 / 6} \nu^{1 / 6}
$$

in which viscosity and resistivity are of equal importance in the layer but inertia is negligible. 


\section{Low viscosity solutions}

The low viscosity ordering of layer parameters $S \nu \ll 1$ (i.e. $\tau_{R} \ll \tau_{V}$ ) is only relevant to the relatively cold edge regions of tokamak plasmas. In the limit $V^{3} S \gg 1$, Eq. (B.2) can be transformed into Eq. (B.8) with $a \sim\left(V^{-3} / \nu\right)^{1 / 2} \gg 1$. It follows that in this limit the dispersion relation reduces to the ideal-inertial result.

Consider the limit $V^{3} S \ll 1$, in which Eq. (B.2) reduces to (B.13) for $k \leqslant \mathcal{O}(1)$, with the solution (B.14). For $k \gtrsim \mathcal{O}(1)$, Eq. (B.2) takes the form

$$
\frac{d^{2} \hat{\phi}}{d u^{2}}=-\mathrm{i} u^{2} \hat{\phi}+\left(\frac{S^{1 / 2} \nu}{V^{3 / 2}}\right) u^{4} \widehat{\phi},
$$

where $u=\left(V^{3} S\right)^{1 / 4} k$. In the limit $S^{1 / 2} \nu / V^{3 / 2} \gg 1$, Eq. (B.18) can be transformed into Eq. (B.15) and the dispersion relation reduces to the visco-resistive form. In the limit $S^{1 / 2} \nu / V^{3 / 2} \ll 1$, Eq. (B.18) can be transformed into the standard Whittaker form, and the solution which is regular as $u \rightarrow \infty$ has the small- $u$ asymptotic behavior

$$
\widehat{\phi} \sim 1-\exp (-\mathrm{i} \pi / 8) \frac{2 \Gamma\left(\frac{3}{4}\right)}{\Gamma\left(\frac{1}{4}\right)}\left(V^{3} S\right)^{1 / 4} k+\cdots .
$$

Equations (B.5)-(B.7), (B.14), and (B.19) yield the well-known 'resistive-inertial' result, ${ }^{8}$

$$
\Delta^{ \pm}=-\exp (\mathrm{i} 3 \pi / 8) 2 \pi \frac{\Gamma\left(\frac{3}{4}\right)}{\Gamma\left(\frac{1}{4}\right)} V^{5 / 4} S^{3 / 4},
$$

in which resistivity and inertia are important in the layer but viscosity is negligible.

\section{Summary and discussion}

In an incompressible plasma tearing and twisting parity modes have the same dispersion relation, so that $\Delta^{+}=\Delta^{-}$. The high viscosity results, valid when $S \nu \gg 1$, are

$$
\begin{aligned}
& \text { (I) } \quad \Delta^{ \pm}=-\mathrm{i} \frac{\pi}{V} \quad|V| \gg \nu^{1 / 3}, \\
& \text { (II) } \quad \Delta^{ \pm}=-4.647 \exp [\mathrm{i} \operatorname{sgn}(V) \pi / 8](|V| \nu)^{-1 / 4} \quad \nu^{1 / 3} \gg|V| \gg S^{-2 / 3} \nu^{-1 / 3} \text {, } \\
& \text { (III) } \quad \Delta^{ \pm}=-2.104 \mathrm{i} V S^{5 / 6} \nu^{1 / 6} \quad S^{-2 / 3} \nu^{-1 / 3} \gg|V| .
\end{aligned}
$$


The low viscosity results, valid when $S \nu \ll 1$, are

$$
\begin{aligned}
\text { (I) } & \Delta^{ \pm}=-\mathrm{i} \frac{\pi}{V} & |V| \gg S^{-1 / 3}, \\
\text { (IV) } & \Delta^{ \pm}=-2.124 \exp [\mathrm{i} \operatorname{sgn}(V) 3 \pi / 8]|V|^{5 / 4} S^{3 / 4} & S^{-1 / 3} \gg|V| \gg S^{1 / 3} \nu^{2 / 3}, \\
\text { (III) } & \Delta^{ \pm}=-2.104 \mathrm{i} V S^{5 / 6} \nu^{1 / 6} & S^{1 / 3} \nu^{2 / 3} \gg|V| .
\end{aligned}
$$

The various regimes are termed the ideal-inertial (I), ideal-viscous (II), visco-resistive (III), and resistive-inertial (IV). The layer widths are given by

$$
\begin{aligned}
\text { (I) } & \frac{\delta_{\text {layer }}}{r_{s}} \sim|V|, \\
\text { (II) } & \frac{\delta_{\text {layer }}}{r_{s}} \sim(|V| \nu)^{1 / 4}, \\
\text { (III) } & \frac{\delta_{\text {layer }}}{r_{s}} \sim\left(\frac{\nu}{S}\right)^{1 / 6}, \\
\text { (IV) } & \frac{\delta_{\text {layer }}}{r_{s}} \sim\left(\frac{|V|}{S}\right)^{1 / 4} .
\end{aligned}
$$

The constant- $\psi$ approximation ${ }^{8}$ is valid whenever the resistive diffusion rate across the layer exceeds the Doppler shifted mode frequency $\omega^{\prime}$. This yields the criterion

$$
\left|\omega^{\prime}\right| \tau_{R}\left(\frac{\delta_{\text {layer }}}{r_{s}}\right)^{2} \equiv|V| S\left(\frac{\delta_{\text {layer }}}{r_{s}}\right)^{2} \ll 1
$$

for the validity of the approximation. It is easily demonstrated that regimes (III) and (IV) are constant- $\psi$ using Eqs. (B.21)-(B.24).

In Fig. 4 the boundaries of the various regimes are plotted in $P-Q$ space, where $P=S \nu$ and $Q=S^{1 / 3}|V|$. In ohmically heated tokamaks $\left(P \sim 10, \omega^{\prime} \sim \omega_{* \mathrm{e}}\right)$ resonant layers usually lie in the visco-resistive regime. In tokamaks heated by unbalanced Neutral Beam Injection (NBI) relative rotation velocities can become sufficiently large to allow layers to enter the ideal-viscous regime.$^{19}$ In edge plasmas the viscosity parameter $P$ becomes less than unity but generally remains large enough to prevent layers from entering the resistive-inertial regime. 


\section{Compressible single-fluid layer theory}

\section{The inviscid dispersion relation}

In cylindrical geometry (i.e. neglecting field-line curvature and Pfirsch-Schlüter effects) the tearing parity dispersion relation in an inviscid, compressible, single-fluid plasma is written ${ }^{41}$

$$
\Delta^{+}=-2.124 \exp [\mathrm{i} \operatorname{sgn}(V) 3 \pi / 8]|V|^{5 / 4} S^{3 / 4} \text {. }
$$

This is equivalent to the resistive-inertial limit of Sec. B.I, which is the relevant limit for resistive modes in an inviscid plasma. It is concluded that plasma compressibility does not affect the dispersion relation for tearing parity modes.

The twisting parity dispersion relation in an inviscid, compressible, single-fluid plasma takes the form ${ }^{41,42}$

$$
\frac{\Delta^{-}}{\Delta^{c}}=-\exp [i \operatorname{sgn}(V) 3 \pi / 8]\left(\frac{|V|}{V_{c}}\right)^{5 / 4} /\left[1-\frac{\Gamma\left(\frac{1}{4}+\frac{1}{4} Q\right)}{\Gamma\left(\frac{1}{4}\right)} \frac{\Gamma\left(\frac{3}{4}\right)}{\Gamma\left(\frac{3}{4}+\frac{1}{4} Q\right)}\right]
$$

in cylindrical geometry, where

$$
\begin{aligned}
\Delta^{c} & =2.124 \beta_{t}^{5 / 6} S^{1 / 3}, \\
V_{c} & =\frac{\beta_{t}^{2 / 3}}{S^{1 / 3}} \\
Q & =\exp [-\mathrm{i} \operatorname{sgn}(V) 3 \pi / 4]\left(\frac{|V|}{V_{c}}\right)^{3 / 2}, \\
\beta_{t} & =\frac{\gamma_{s} \mu_{0} p\left(r_{s}\right)}{B_{0}^{2}}
\end{aligned}
$$

Here, $p(r)$ is the plasma pressure and $\gamma_{s}$ the usual ratio of specific heats.

The dispersion relation (B.26) has two distinct asymptotic limits:

$$
\begin{array}{ll}
\Delta^{-} \simeq-2.124 \operatorname{erp}[i \operatorname{sgn}(V) 3 \pi / 8]|V|^{5 / 4} S^{3 / 4} & |V| \gg V_{c}, \\
\Delta^{-} \simeq 2.704 \exp [i \operatorname{sgn}(V) \pi / 8] \frac{\beta_{t} S^{1 / 4}}{|V|^{1 / 4}} & |V| \ll V_{c} .
\end{array}
$$


So, for $|V| \gg V_{c},(\mathrm{~B} .26)$ asymptotes to the tearing parity dispersion relation (B.25). On the other hand, for $|V| \ll V_{c}$, (B.26) reduces to a typical resistive interchange dispersion relation. ${ }^{10}$ It can be demonstrated that the imaginary part of $\Delta^{-}$is zero when $|V|=0.5615 V_{c}$ and $\operatorname{Re}\left(\Delta^{-}\right)=1.130 \Delta^{c}$. This corresponds to the marginal stability point for the undriven interchange mode. For $\Delta^{-}<1.130 \Delta^{c}$ there are no intrinsically unstable roots of (B.26), whereas for $\Delta^{-}>1.130 \Delta^{c}$ the resistive interchange mode becomes intrinsically unstable with a typical growth-rate $\gamma \tau_{H} \sim V_{c}$.

Equations (B.28) suggest that the response of a twisting parity layer to a rotating external magnetic perturbation is similar to that of a tearing parity layer, except when the imposed frequency lies within a typical interchange growth-rate of the natural frequency (i.e. $|V|<$ $\left.V_{c}\right)$, in which case the response is greatly modified. This modification is such as to ensure that $\left|\Delta^{-}\right|$never falls significantly below the critical twisting parity stability index needed to destabilize the resistive interchange mode (i.e. $\left|\Delta^{-}\right| \gtrsim \Delta^{c}$ ).

The twisting parity dispersion relation can be modelled by writing

$$
\Delta^{-}=\Delta^{+}+\Delta^{c}
$$

where $\Delta^{+}$is given by (B.25) and $\Delta^{c}$ by (B.27a). This formula reproduces all of the salient features of the true dispersion relation. For instance, it yields $\Delta^{-} \simeq \Delta^{+}$for $|V| \gg V_{c}$, with $V_{c}$ given by (B.27b). It also implies that the marginal stability point of the resistive interchange mode is $\Delta^{-}=\Delta^{c}$, with $\Delta^{-}>\Delta^{c}$ needed for instability. Finally, (B.29) suggests that $\left|\Delta^{-}\right| \geqslant \Delta^{c}$ for an externally driven twisting parity layer as the driving frequency is scanned across the natural frequency.

\section{The viscous dispersion relation}

It is not possible to obtain an exact analytic twisting parity dispersion relation for a viscous, compressible plasma. ${ }^{41}$ However, the model dispersion relation (B.29) can be generalized to 
take viscosity into account using the following arguments. The usual criterion for the neglect of plasma compressibility in resonant layers is

$$
\omega^{\prime} \gg k_{\|} c_{s}
$$

where $k_{\|}$is the typical parallel wavenumber, and $c_{s} \equiv \sqrt{\gamma_{s} p / \rho}$ is the sound speed. Equation (B.30) can be shown to reduce to

$$
|V| \gg V_{c} \equiv \sqrt{\beta_{t}} \frac{\delta_{\text {layer }}}{r_{s}} .
$$

Thus, in the ideal-viscous (II), visco-resistive (III), and resistive-inertial (IV) regimes:

$$
\begin{aligned}
& \text { (II) } \quad V_{c}=\beta_{t}^{2 / 3} \nu^{1 / 3}, \\
& \text { (III) } \quad V_{c}=\beta_{t}^{1 / 2} \frac{\nu^{1 / 6}}{S^{1 / 6}}, \\
& \text { (IV) } \quad V_{c}=\frac{\beta_{t}^{2 / 3}}{S^{1 / 3}},
\end{aligned}
$$

where use has been made of Eqs. (B.23). Note the identity of Eqs. (B.27.b) and (B.32c). Clearly, the parameter $V_{c}$ in Sec. B.II.1 is equivalent to the (normalized) mode frequency above which compressibility effects are negligible. Thus, (B.28a) is the incompressible twisting mode dispersion relation [note that this is equivalent to the tearing mode dispersion relation (B.25), as hypothesized in Sec. B.I.1], and (B.28b) is the related compressible dispersion relation.

A comparison of Eqs. (B.25), (B.27a), and (B.27b) shows that the critical stability index, $\Delta^{c}$, needed to destabilize the resistive interchange mode in the resistive-inertial regime (IV) is equal to $\left|\Delta^{+}\left(V_{c}\right)\right|$, where $\Delta^{+}$is the tearing mode (or incompressible twisting mode) dispersion relation. It is assumed that this equality holds in all regimes, which yields the generalized result:

$$
\text { (II) } \quad \Delta^{c}=\frac{4.647}{\beta_{t}^{1 / 6} \nu^{1 / 3}}
$$




$$
\begin{aligned}
& \text { (III) } \quad \Delta^{c}=2.104 \beta_{t}^{1 / 2} S^{2 / 3} \nu^{1 / 3}, \\
& \text { (IV) } \quad \Delta^{c}=2.124 \beta_{t}^{5 / 6} S^{1 / 3},
\end{aligned}
$$

where use has been made of Eqs. (B.21) and (B.22). It is easily demonstrated that Eqs. (B.32c) and (B.33c) are valid when $S \nu \ll \beta_{t}$, Eqs. (B.32b) and (B.33b) when $\beta_{t} \ll S \nu \ll 1 / \beta_{t}$, and Eqs. (B.32a) and (B.33a) when $1 / \beta_{t} \ll S \nu$. In a typical ohmically heated tokamak plasma $S \nu \sim 10$, so (B.32b) and (B.33b) are the most physically relevant critical parameters. ${ }^{19}$ The boundary between compressible and incompressible layer physics (i.e. $|V|=V_{c}$ ) is plotted in Fig. 4. It can be seen that ideal-inertial (I) layers are always incompressible.

Compressible layers are subject to the well known Pfirsch-Schlüter enhancement of inertia (by $1+2 q^{2}$ ) in toroidal geometry. ${ }^{16}$ This implies that

$$
\begin{gathered}
\tau_{H} \rightarrow \sqrt{1+2 q^{2}\left(r_{s}\right)} \tau_{H}, \\
\beta_{t} \rightarrow\left[1+2 q^{2}\left(r_{s}\right)\right] \beta_{t},
\end{gathered}
$$

for $|V|<V_{c}$. In fact, $V_{c}$ is usually quite small compared to a typical diamagnetic frequency (see Sec. VI), so the enhancement of inertia is generally unimportant when dealing with the interaction of layers possessing widely dispersed natural frequencies.

\section{Two-fluid layer theory}

The most important consequence of introducing two-fluid effects into the inner region is to cause the various different types of instability to propagate at different frequencies. According to Coppi ${ }^{33}$ tearing modes propagate at the $\mathbf{E} \wedge \mathbf{B}$ plus the electron diamagnetic frequency, resistive interchange modes at the $\mathbf{E} \wedge \mathbf{B}$ frequency, and inertia dominated (i.e. ideal) modes at the $\mathbf{E} \wedge \mathbf{B}$ plus the ion diamagnetic frequency. Thus, the natural frequency of tearing parity resistive modes (i.e. the propagation frequency of uncoupled drift tearing modes) is 
likely to differ from the natural frequency of twisting parity resistive modes (i.e. the propagation frequency of uncoupled resistive interchange modes) by a typical electron diamagnetic frequency. This statement is, of course, a gross oversimplification but is, nevertheless, in general agreement with conclusions drawn from detailed stability calculations for tearing and twisting parity drift modes. See, for example, Figs. 4 and 9 in Finn et al.,$^{43}$ Fig. 4 in Kim et al. ${ }^{44}$ and Figs. 2 and 5 in Kim and Choi. ${ }^{45}$

\section{Conclusions}

In a typical ohmically heated tokamak plasma the response of a tearing parity layer to a rotating external magnetic perturbation is governed by the visco-resistive dispersion relation of Sec. B.I, so that

$$
\Delta^{+}(\omega)=-2.104 \mathrm{i}\left(\omega-\omega_{0}^{+}\right) \frac{\tau_{H}^{1 / 3} \tau_{R}^{5 / 6}}{\tau_{V}^{1 / 6}},
$$

where $\omega$ is the applied frequency, $\omega_{0}^{+}$is the natural frequency for tearing parity modes, and $\tau_{H}, \tau_{R}$, and $\tau_{V}$ are the local hydromagnetic, resistive, and viscous time-scales, respectively (see Sec. B.I.1). The natural frequency $\omega_{0}^{+}$is the propagation frequency of the uncoupled drift tearing mode, and is determined by local equilibrium fluid flows.

In a typical ohmically heated tokamak plasma the response of twisting parity layer to a rotating external magnetic perturbation is well approximated by

$$
\Delta^{-}(\omega)=-2.104 \mathrm{i}\left(\omega-\omega_{0}^{-}\right) \frac{\tau_{H}^{1 / 3} \tau_{R}^{5 / 6}}{\tau_{V}^{1 / 6}}+\Delta^{c},
$$

where

$$
\Delta^{c}=2.104 \beta_{t}^{1 / 2} \frac{\tau_{R}^{2 / 3}}{\tau_{H}^{1 / 3} \tau_{V}^{1 / 3}}
$$

is the critical twisting parity stability index needed to destabilize the resistive interchange mode. The parameter $\beta_{t}$ is a measure of the stabilizing effect of plasma compressibility [see (B.27d)]. In Eq. (B.36), $\omega_{0}^{-}$is the natural frequency for twisting parity modes (i.e. the prop- 
agation frequency of the uncoupled resistive interchange mode). In general, the difference between $\omega_{0}^{+}$and $\omega_{0}^{-}$is of order the local electron diamagnetic frequency. 


\section{References}

1. J.A. Wesson, R.D. Gill, M. Hugon, F.C. Schüler, J.A. Snipes, D.J. Ward, D.V. Bartlett, D.J. Campbell, P.A. Duperrex, A.W. Edwards, R.S. Granetz, N.A.O. Gottardi, T.C. Hender, E. Lazzaro, P.J. Lomas, N. Lopes Cardozo, K.F. Mast, M.F.F. Nave, N.A. Salmon, P. Smeulders, P.R. Thomas, B.J.D. Tubbing, M.F. Turner, and A. Weller, Nucl. Fusion 29, 641 (1989).

2. P.H. Rebut, D. Boucher, D.J. Gambier, B.E. Keen, and M.L. Watkins, Fusion Technology 1992, Proceedings of the 17th Symposium on Fusion Technology, Rome (NorthHolland, Amsterdam, 1993), Vol. 1, p. 7.

3. B.A. Carreras, P.H. Diamond, M. Murakami, J.L. Dunlap, J.D. Bell, H.R. Hicks, J.A. Holmes, E.A. Lazarus, V.K. Paré, P. Similon, C.E. Thomas, and R.M. Wieland, Phys. Rev. Letts. 50, 503 (1983).

4. R. Fitzpatrick, R.J. Hastie, T.J. Martin, and C.M. Roach, Nucl. Fusion 33, 1533 (1993).

5. J.W. Connor, R.J. Hastie, and J.B. Taylor, Proc. R. Soc. London Ser. A 365, 1 (1978).

6. J.W. Connor, R.J. Hastie, and J.B. Taylor, Phys. Fluids B 3, 1539 (1991).

7. J.W. Connor, S.C. Cowley, R.J. Hastie, T.C. Hender, A. Hood, and T.J. Martin, Phys. Fluids 31, 577 (1988).

8. H.P. Furth, J. Killeen, and M.N. Rosenbluth, Phys. Fluids 6, 459 (1963).

9. W.A. Newcomb, Ann. Phys. 10, 232 (1960).

10. B. Coppi, J.M. Greene, and J.L. Johnson, Nucl. Fusion 6, 101 (1966). 
11. C. Mercier, Nucl. Fusion 1, 47 (1960).

12. R.C. Grimm, R.L. Dewar, J. Manickam, S.C. Jardin, A.H. Glasser, and M.S. Chance, in Plasma Physics and Controlled Nuclear Fusion Research 1982, Proceedings of the 9th International Conference, Baltimore (IAEA, Vienna, 1983), Vol. 3, p. 35.

13. R.L. Dewar, and M. Persson, Phys. Fluids B 5, 4273 (1993).

14. M.N. Bussac, D. Edery, R. Pellat, and J.L. Soulé, in Plasma Physics and Controlled Nuclear Fusion Research 1976, Proceedings of the 6th International Conference, Berchtesgaden (IAEA, Vienna, 1977), Vol. 1, p. 607.

15. S.C. Cowley, and R.J. Hastie, Phys. Fluids 31, 426 (1988).

16. A.H. Glasser, J.M. Greene, and J.L. Johnson, Phys. Fluids 18, 875 (1975).

17. J.F. Drake, and Y.C. Lee, Phys. Fluids 20, 1341 (1977).

18. R. Fitzpatrick, Phys. Fluids B 2, 2636 (1990).

19. R. Fitzpatrick, Nucl. Fusion 33, 1049 (1993).

20. R. Fitzpatrick, in Theory of Fusion Plasmas, Proceedings of the Joint Varenna-Lausanne International Workshop, Varenna 1992, (Società Italiana di Fisica, Bologna, 1992), p. 147.

21. R.J. La Haye, A.W. Hyatt, and J.T. Scoville, Nucl. Fusion 32, 2119 (1992).

22. R.B. White, D.A. Monticello, M.N. Rosenbluth, and B.V. Waddell, Phys. Fluids 3, 1532 (1991).

23. J.W. Connor, R.J. Hastie, and J.B. Taylor, Phys. Fluids B 3, 1532 (1991). 
24. D. Edery, J.L. Soulé, R. Pellat, M. Frey, and J.P. Somon, in Plasma Physics and Controlled Nuclear Fusion Research 1980, Proceedings of the 8th International Conference, Brussels (IAEA, Vienna, 1981), Vol. 1, p. 269.

25. W. Gautschi, and W.F. Cahill, in Handbook of Mathematical Tables, Eds. M. Abramowitz, and I.A. Stegun (Dover, New York, 1965), Chap. 5.

26. J.W. Connor, R.J. Hastie, and H.R. Wilson, Phys. Fluids B 4, 56 (1992).

27. T.C. Hender, R. Fitzpatrick, A.W. Morris, P.G. Carolan, R.D. Durst, T. Edlington, J. Ferreira, S.J. Fielding, P.S. Haynes, J. Hugill, I.J. Jenkins, R.J. La Haye, B.J. Parham, D.C. Robinson, T.N. Todd, M. Valovič, and G. Vayakis, Nucl. Fusion 32, 2091 (1992).

28. J.T. Scoville, R.J. La Haye, A.G. Kellman, T.H. Osborne, R.D. Stambaugh, E.J. Strait, and T.S. Taylor, Nucl. Fusion 31, 875 (1991).

29. R. Toschi, in Plasma Physics and Controlled Nuclear Fusion Research 1990, Proceedings of the 13th International Conference, Washington (IAEA, Vienna, 1991), Vol. 3, p. 225.

30. M. Huguet, in Steady-State Operation of Fusion Plasmas, Proceedings of the 11th European Tokamak Workshop, Noordwijk (FOM-Nieuwegein, 1993), p. 547.

31. D.E. Post, K. Borrass, J.D. Callen, S.A. Cohen, J.G. Cordey, F. Engelmann, N. Fujisawa, M.F.A. Harrison, T.J. Hogan, H.J. Hopman, Y. Igitkhanov, O. Kardaun, S.M. Kaye, S. Krasheninnikov, A. Kukushkin, V. Mukhovatov, W.M. Nevins, A. Nocentini, H.W. Pacher, V.V. Parail, L.D. Pearlstein, L.J. Perkins, S. Putvinskij, K. Riedel, D.J. Sigmar, M. Sugihara, D.W. Swain, T. Takizuka, K. Tani, T. Tsunematsu, N.A.Uckan, 
J.G. Wegrowe, J. Wesley, S. Yamamoto, R. Yoshino, K. Young, and P.N. Yushmanov, in ITER Physics, ITER Document Series No. 21 (IAEA, Vienna, 1991).

32. R. Fitzpatrick, and: C. Hender, Phys. Fluids B 3, 644 (1991).

33. B. Coppi, Phys. Fluids 7, 1501 (1964).

34. P.H. Rutherford, Phys. Fluids 16, 1903 (1973).

35. M.N. Bussac, R. Pellat, D. Edery, and J.L. Soulé, Phys. Rev. Lett. 35, 1638 (1975).

36. G. Ara, B. Basu, B. Coppi, G. Laval, M.N. Rosenbluth, and B.V. Waddell, Ann. Phys. (NY) 112, 443 (1978).

37. F. Porcelli, Phys. Fluids 30, 1734 (1987).

38. K. Brau, M. Bitter, R.J. Goldston, D. Manos, K. McGuire, and S. Suckewer, Nucl. Fusion 23, 1643 (1983).

39. J.C.P. Miller, in Handbook of Mathematical Tables, Eds. M. Abramowitz and I.A. Stegun (Dover, New York, 1965), Chap. 19.

40. A. Bondeson, and J.R. Sobel, Phys. Fluids 27, 2028 (1984).

41. J.W. Connor, R.J. Hastie, T.J. Martin, A. Sykes, and M.F. Turner, in Plasma Physics and Controlled Nuclear Fusion Research 1982, Proceedings of the 9th International Conference, Baltimore (IAEA, Vienna, 1983), Vol. 3, p. 403.

42. J.F. Drake, and T.M. Antonsen, Jr., Phys. Fluids 28, 544 (1985).

43. J.M. Finn, W.M. Manheimer, and T.M. Antonsen, Jr., Phys. Fluids 26, 962 (1983).

44. J-Y. Kim, G-S. Cho, and D-I. Choi, Phys. Fluids 31, 2659 (1988).

45. J-Y. Kim, and D-I. Choi, Phys. Fluids B 1, 1026 (1989). 


\section{Figure Captions}

1. Bifurcation curves for locking to the tearing and twisting resonances at a general rational surface. $y^{+}$[defined in Eq. (42d)] is the normalized locking torque due to the tearing resonance, and $y^{-}$[defined in Eq. (42e)] is the normalized locking torque due to the twisting resonance. The solid curves correspond to locking to the tearing resonance, whereas the dashed curves correspond to locking to the twisting resonance. Curves are shown for various different values of the unperturbed normalized frequency $f_{1}$ [defined in Eq. (42b)].

2. The plasma displacements associated with a high- $n$ global resistive instability before (a) and after (b) the bifurcation. The dashed lines represent rational surfaces. Before the bifurcation all the rational surfaces possess local twisting parity. After the bifurcation alternate rational surfaces possess local tearing parity (outside the resistive layers), leading to the formation of interlocking mapnetic islands. In both cases the structure repeats after every fourth rational sur

3. The plasma displacements associated with a high- $n$ global resistive instability in the limit where the difference between the natural frequencies of tearing and twisting modes is negligible. The dashed lines represent rational surfaces. All of the rational surfaces possess local tearing parity (outside the resistive layers), leading to the formation of magnetic islands on every surface. The structure repeats after every fourth rational surface.

4. Boundaries of the various layer regimes in $P-Q$ space, where $P=S \nu$ and $Q=S^{1 / 3}|V|$ [see Eqs. (B.4)]. The solid lines are the boundaries between the major regimes: i.e. the ideal-inertial (I), ideal-viscous (II), visco-resistive (III), and resistive-inertial (IV). The 
dashed line represents the boundary between compressible and incompressible layer physics (compressible layer physics lies on the low- $Q$ side of this line). 


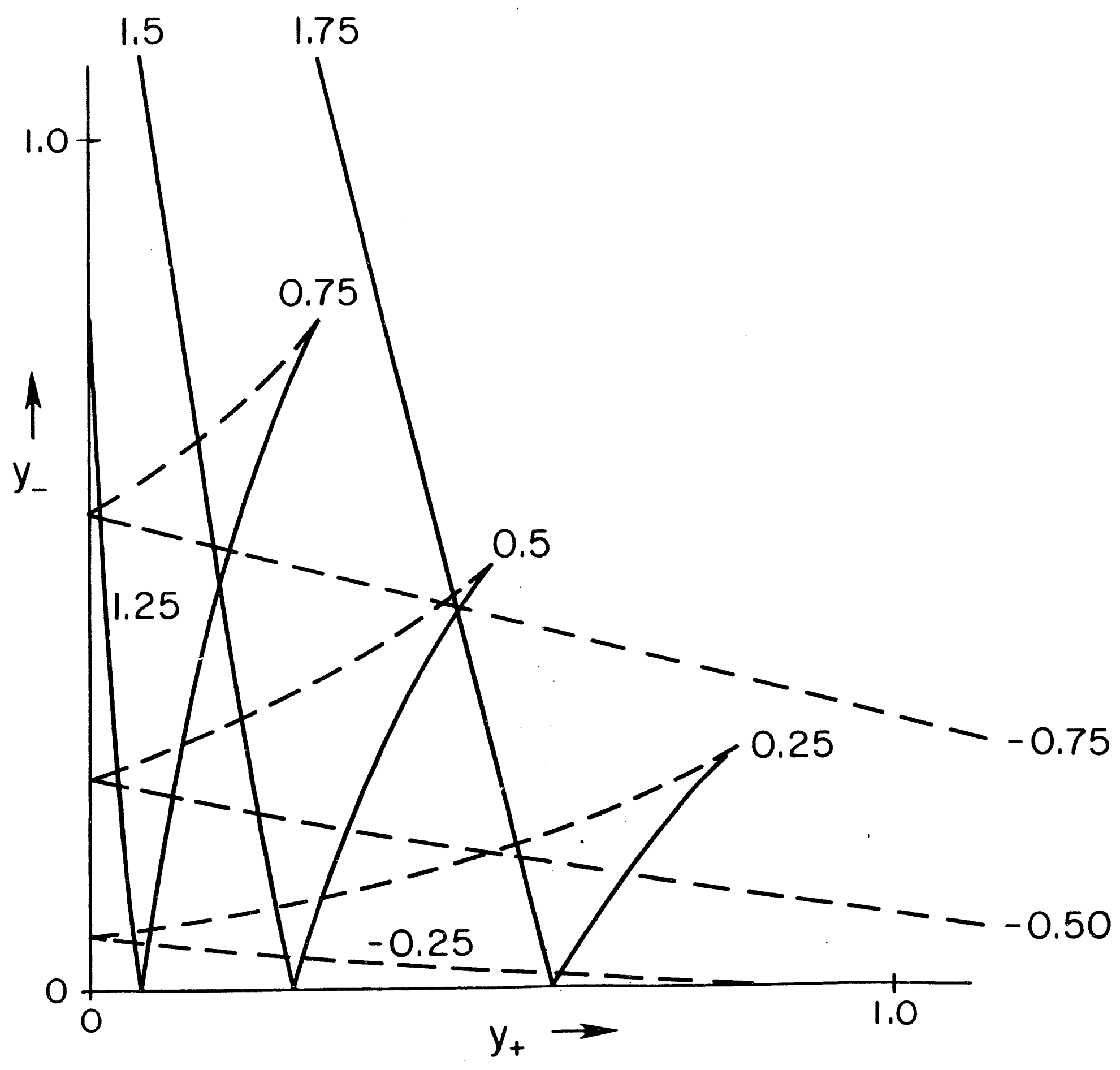




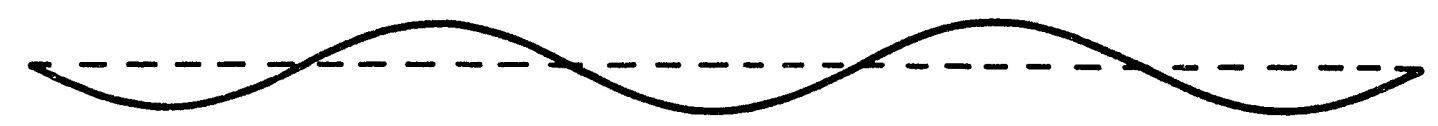

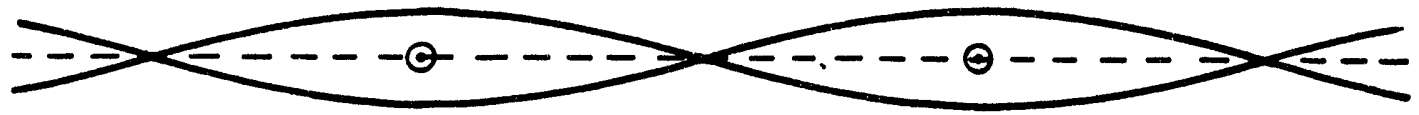

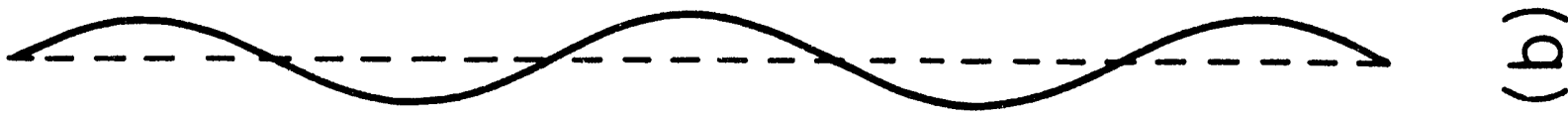

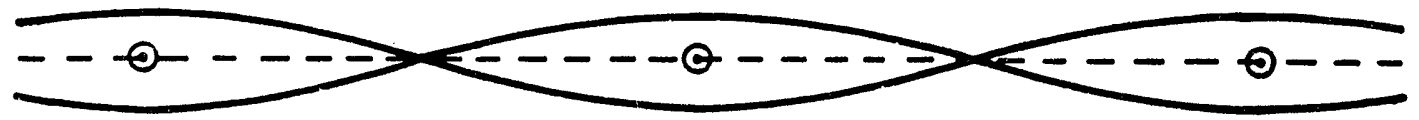

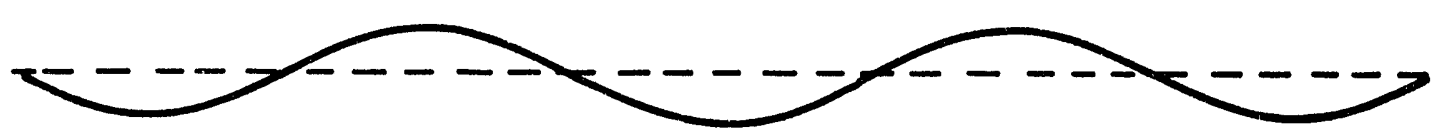

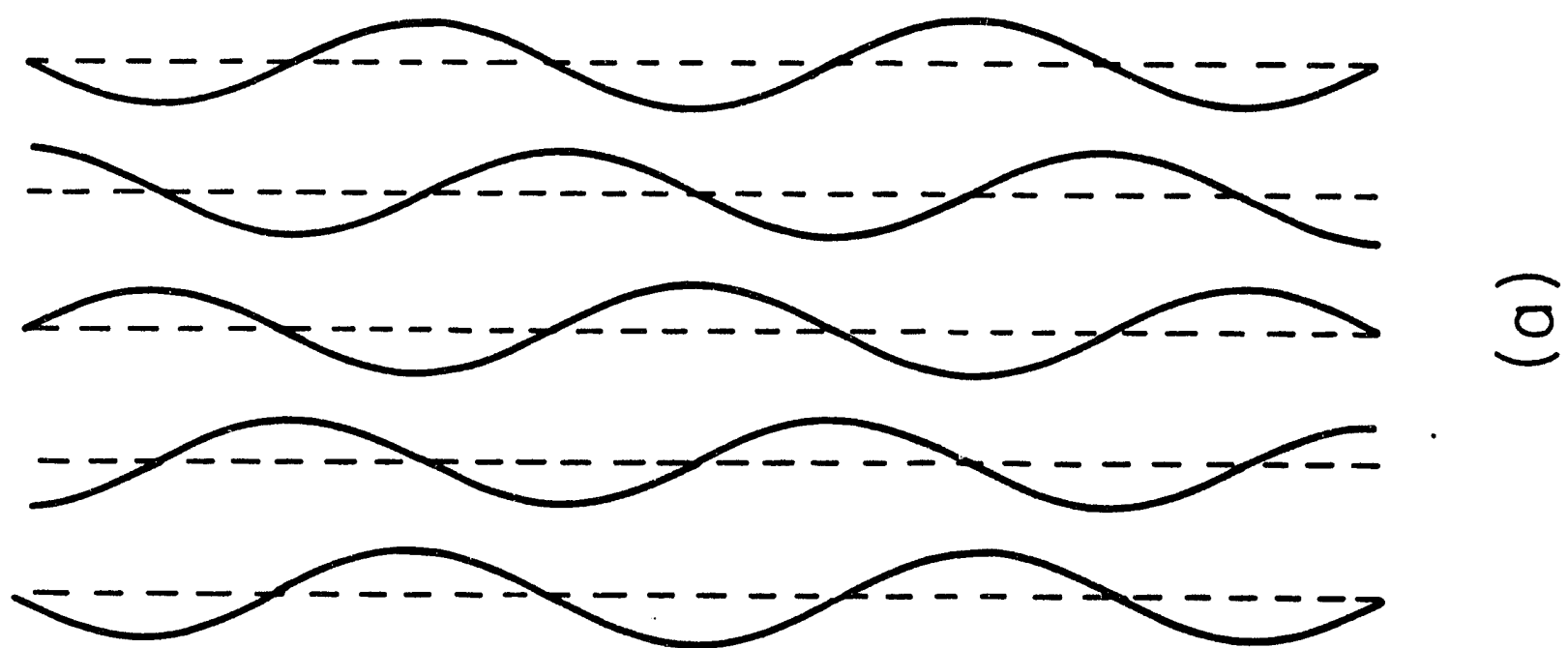




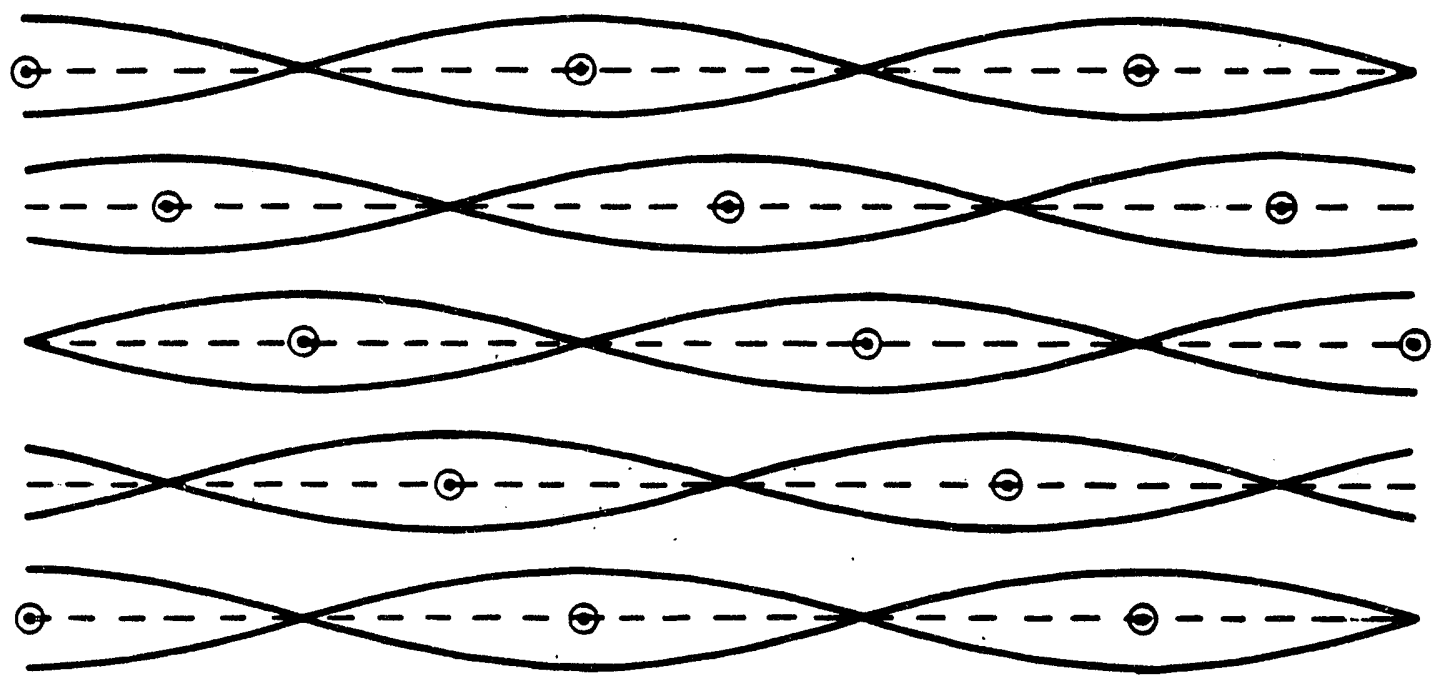




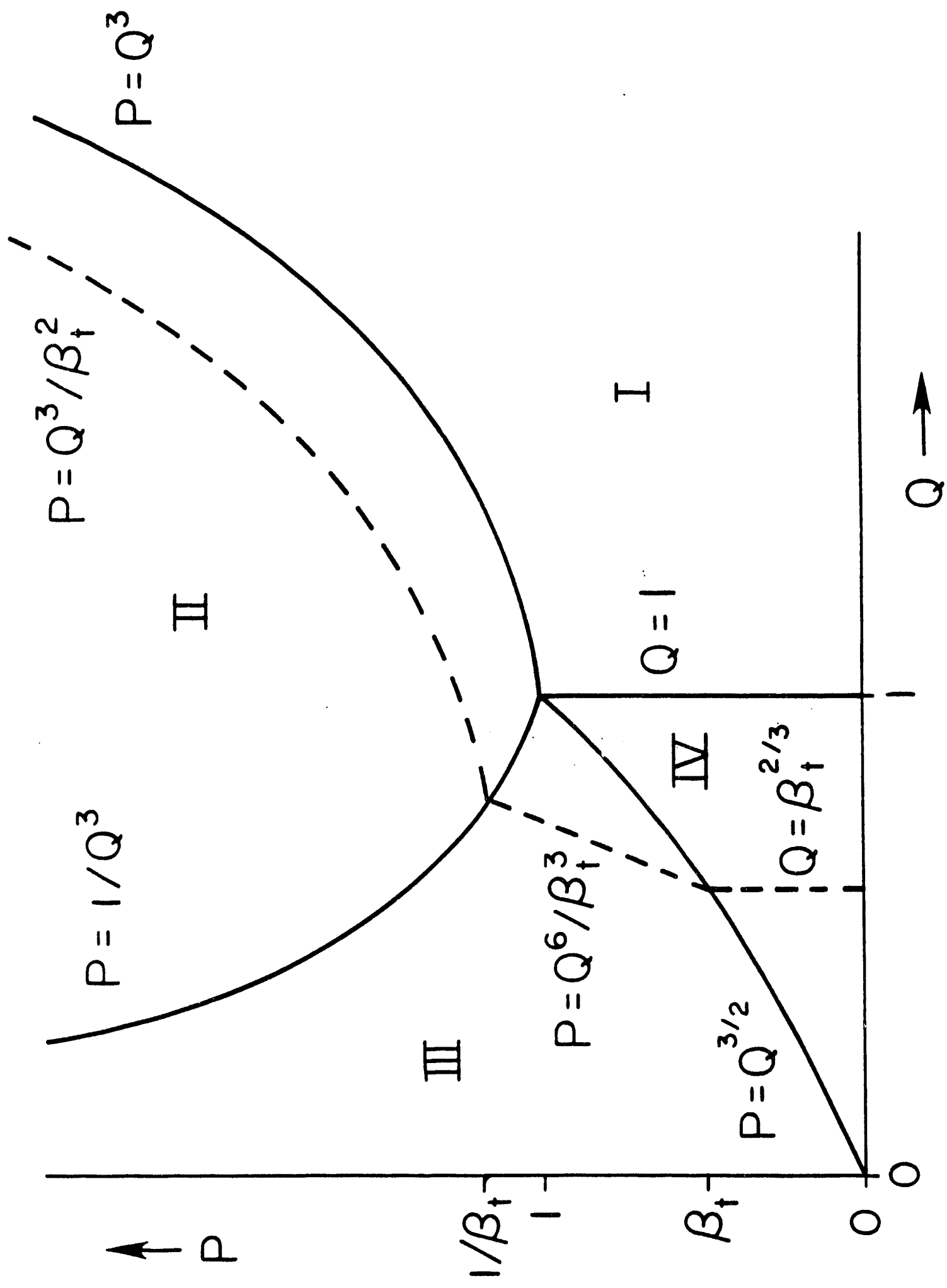



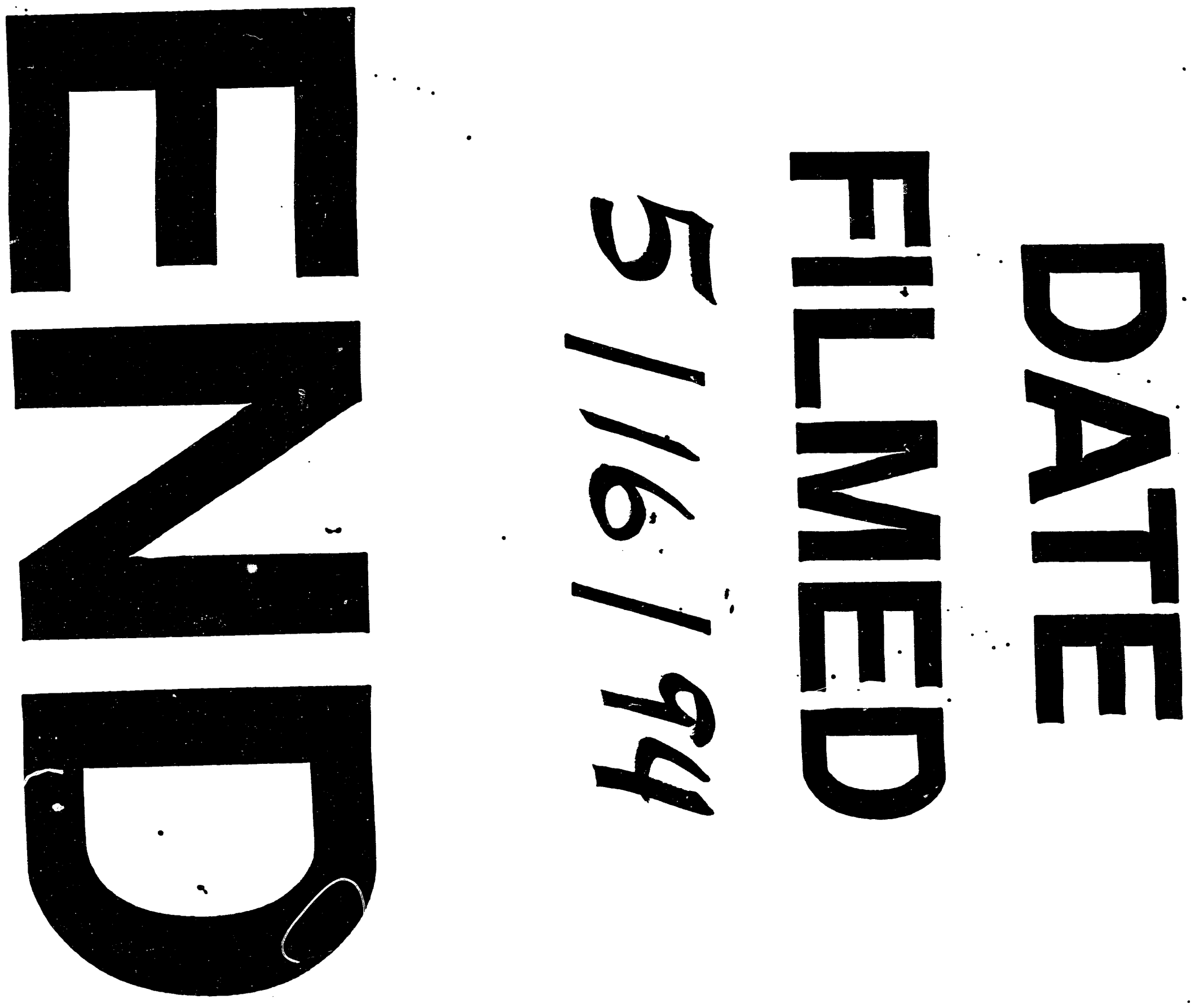


$$
\longrightarrow
$$

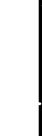

